Joint multizonal transdimensional Bayesian inversion of surface wave dispersion and ellipticity curves for local near-surface imaging

\author{
Journal Article \\ Author(s): \\ Halló, Miroslav (1D; Imperatori, Walter; Panzera, Francesco (1); Fäh, Donat \\ Publication date: \\ 2021-07 \\ Permanent link: \\ https://doi.org/10.3929/ethz-b-000500533 \\ Rights / license: \\ In Copyright - Non-Commercial Use Permitted \\ Originally published in: \\ Geophysical Journal International 226(1), https://doi.org/10.1093/gji/ggab116
}




\title{
Joint multizonal transdimensional Bayesian inversion of surface wave dispersion and ellipticity curves for local near-surface imaging
}

\author{
Miroslav Hallo ${ }^{\circledR}$, Walter Imperatori, Francesco Panzera and Donat Fäh \\ Swiss Seismological Service,ETH Zurich, Zürich,8092, Switzerland.E-mail: miroslav.hallo@sed.ethz.ch
}

Accepted 2021 0. Received 2021 March 5; in original form 2020 October 6

\begin{abstract}
SUMMAR Y
Physical properties of near-surface soil and rock layers play a fundamental role in the seismic site effects analysis, being an essential element of seismic hazard assessment. Site-specific mechanical properties (i.e. shear- and compressional-wave velocities and mass density) can be inferred from surface wave dispersion and horizontal-to-vertical or ellipticity data by nonlinear inversion techniques. Nevertheless, results typically exhibit significant inherent nonuniqueness as different models may fit the data equally well. Standard optimization inversion techniques minimize data misfit, resulting in a single representative model, rejecting other models providing similar misfit values. An alternative inversion technique can be formulated in the Bayesian framework, where the posterior probability density on the model space is inferred. This paper introduces an inversion approach of surface wave dispersion and ellipticity data based on a novel multizonal transdimensional Bayesian formulation. In particular, we parametrize 1-D layered velocity models by the varying number of Voronoi nuclei, allowing us to treat the number of layers as an unknown parameter of the inverse problem. The chosen parametrization leads to the transdimensional formulation of the model space, sampled by a reversible jump Markov chain Monte Carlo algorithm to provide an ensemble of random samples following the posterior probability density of model parameters. The used type of the sampling algorithm controls a model complexity (i.e. the number of layers) self-adaptively based on the measured data's information content. The method novelty lies in the parsimonious selection of sampling models and in the multizonal formulation of prior assumptions on model parameters, the latter allows including additional site-specific constraints in the inversion. These assumptions may be based on, e.g. stratigraphic logs, standard penetration tests, known water table, and bedrock depth. The multizonal formulation fully preserves the validity of the transdimensional one, as demonstrated analytically. The resultant ensemble of model samples is a discrete approximation of the posterior probability density function of model parameters and associated properties (e.g. $V_{\mathrm{S} 30}$, quarter-wavelength average velocity profile and theoretical SH-wave amplification function). Although the ultimate result is the posterior probability density function, some representative models are selected according to data fit and maximum of the posterior probability density function. We first validate our inversion approach based on synthetic tests and then apply it to field data acquired from the active seismic survey and single-station measurements of ambient vibrations at the SENGL seismic station site in central Switzerland.
\end{abstract}

Key words: Inverse theory; Joint inversion; Probability distributions; Site effects; Surface waves and free oscillations; Crustal structure.

\section{INTRODUCTION}

The local seismic hazard assessment is important for engineering and public safety applications. It is influenced by the structure and physical properties of the uppermost part of the Earth crust, as these affect the local amplification of seismic waves. In this context, a site characterization aims to retrieve shear- and compressional-wave velocities ( $S$ - and $P$-wave velocities, respectively) as well as mass density of near-surface structures (i.e. elastic properties of the medium), because they determine principal local effects on ground motion. In seismic hazard codes, the $S$-wave velocity is an attribute of the primary importance. It is typically expressed in terms of its average value to a depth of $30 \mathrm{~m}\left(v_{\mathrm{S} 30}\right.$; e.g. Borcherdt 1994) or in other alternative forms as the quarter-wavelength velocity profile representation (QWL; e.g. Joyner et al. 1981; 
Boore 2003; Poggi et al. 2011, 2012). The local near-surface structure can be examined directly (e.g. drilling boreholes) or, more commonly, indirectly by inversion of geophysical data. The latter is recognized as an efficient non-invasive way to retrieve information on the subsurface. The geophysical data can be acquired from recorded wavefield excited by active sources or ambient seismic noise. Among others, the recorded wavefield is composed of Rayleigh and Love surface waves reflecting the dispersive properties of the vertically heterogeneous near-surface structure (e.g. Bonnefoy-Claudet et al. 2006).

\subsection{Rayleigh and Love wave data}

In this paper's context, fundamental and higher modes of surface waves are the input data of the inverse modelling. In particular, data of interest are phase velocity dispersion curves of Rayleigh and Love waves and ellipticity curves of Rayleigh waves. These data cannot be measured directly, but they are retrieved from the single-station and seismic array measurements of ambient vibrations or active seismic surveys (e.g. Foti et al. 2018). As measured indirectly, we provide here a brief overview of acquisition techniques.

Single-station methods typically help to obtain a horizontal-to-vertical spectral ratio (H/V; Nogoshi \& Igarashi 1971; Nakamura 1989). The H/V ratio may be related to the Rayleigh wave ellipticity (Yamanaka et al. 1994; Fäh et al. 2001; D'Amico et al. 2008); nevertheless, it is more complex phenomenon as ambient vibrations are made by an unpredictable brew of various wave types present in the wavefield (e.g. Bonnefoy-Claudet et al. 2008). Therefore, a direct inversion of $\mathrm{H} / \mathrm{V}$ curves is possible only when assuming an isotropic wavefield and equipartitioning of the seismic energy among the different wave modes (Sánchez-Sesma et al. 2011). To overcome this issue, single-station techniques to estimate Rayleigh wave ellipticity from the wavefield have been developed (e.g. RayDec method, Hobiger et al. 2009). In the RayDec method, Love waves are suppressed by an averaging process as they are not present on the vertical component, and body waves from distant sources are suppressed as well because they do not have a phase shift between vertical and horizontal signals. Next, the array methods are aimed at the velocity and azimuth of Rayleigh and Love waves to identify the dispersion curves of the fundamental and higher modes (e.g. Aki 1957; Horike 1985; Okada 2003). Recently, Poggi \& Fäh (2010) proposed an array frequency-wavenumber $(f-k)$ processing method for joint estimation of Rayleigh wave ellipticity together with dispersion curves, and Maranò et al. (2012) draw a wavefield decomposition method for iterative separation of contributions of different waves. Apart from these passive methods, an example of an active seismic array method is the multichannel analysis of surface waves (MASW, Park et al. 1999).

\subsection{Inverse modelling of local near-surface structures}

The inversion of surface wave curves (i.e. data) to retrieve a local near-surface structure (i.e. model) is a typical non-linear inverse problem. Primarily, dispersion curves of surface waves depend non-linearly on the vertically heterogeneous $S$-wave velocity structure below the measurement site. Additionally, they depend on the $P$-wave velocity and the mass density of the subsurface structure. There exists a dependence on the anelastic properties of the medium (i.e. material damping), but it has been shown to be of secondary importance (e.g. Lunedei \& Albarello 2009). Linearized inversion procedures were proposed and applied in the past (e.g. Tokimatsu et al. 1991), but several studies have shown that linearized inversions inherently depend on an assumed initial model (e.g. Parolai et al. 2006). Next, the inversion of the Rayleigh wave ellipticity (or alternatively $\mathrm{H} / \mathrm{V}$ spectral ratio) alone leads to a non-unique solution due to trade-offs between the $S$-wave velocity and layer thickness (e.g. Hobiger et al. 2013). Moreover, independent inversions of multiple individual modes of surface waves are not a viable option, leading to multiple different solutions for one site. Therefore, all the available data should be inverted jointly using a non-linear inversion method.

For instance, Scherbaum et al. (2003) proposed a joint inversion of dispersion curves and H/V spectral ratios to retrieve $S$-wave velocity. The inversion was constrained by a simple model consisting of a single sedimentary layer over an elastic half-space. Another non-linear inversion of H/V spectral ratios based on a genetic algorithm technique has been proposed by Fäh et al. (2003), where the authors assumed the thickness of sediments to be known. In Arai \& Tokimatsu (2004), the inversion was constrained by fixing either thickness or $S$-wave velocity of layers, and it is solved by the iterative singular value decomposition. This iterative procedure has been subsequently enhanced into the joint inversion of H/V and dispersion curves (Arai \& Tokimatsu 2005). Wathelet et al. (2004) proposed the inversion of dispersion curves based on the neighbourhood algorithm (Sambridge 1999) to retrieve both $P$ - and $S$-wave velocities. However, Wathelet (2008) emphasized that their model parametrization in terms of $P$-wave velocity and $S / P$-wave velocity ratio leads to non-uniform and biased intrinsic prior of the $S$-wave velocity (i.e. the parameter of the primary importance). Hence, Wathelet (2008) proposed a model parametrization based on separated $S$ - and $P$-wave velocities. The importance of the non-uniqueness and solution uncertainty was tackled by Foti et al. (2009). To this end, the solution uncertainty originates from data and modelling errors that can be treated using a Bayesian (probabilistic) inversion method. For instance, Maraschini \& Foti (2010) proposed a probabilistic inversion of multimodal Rayleigh and Love wave dispersion curves to retrieve separated $S$ and $P$-wave velocities as well as the mass density. They constrained the inversion by a weekly informative prior, and they explore the model space by a Monte Carlo algorithm. The Bayesian inversion of the fundamental Rayleigh wave dispersion curve by Molnar et al. (2010) is based on the Monte Carlo algorithm and it uses a Toeplitz data covariance matrix to account for data errors. Dettmer et al. (2012) proposed a transdimensional Bayesian inversion of the fundamental Rayleigh wave dispersion curve with autoregressive data errors in order to infer the number of layers, $S$-wave velocity, $P / S$-wave velocity ratio and mass density. Li et al. (2012) demonstrated advantages of using a joint 
multimodal inversion by a Bayesian inversion of multimodal Scholte wave dispersion curves in the North Sea. More recently, Cipta et al. (2018) applied a transdimensional Bayesian inversion of the H/V spectral ratio in the Jakarta Basin.

In this work, we propose a joint Bayesian inversion of multimodal Rayleigh and Love wave dispersion curves and Rayleigh wave ellipticity curve (or ellipticity angle). We parametrize the layered model by separated $S$ - and $P$-wave velocities and mass density. Thicknesses of horizontal isotropic layers are parametrized in logarithmic depth, reflecting the reduction of the information contained in the observed data with depth. We define the model space in the Bayesian probabilistic framework (Tarantola 2005) and explore it by the reversible jump Markov chain Monte Carlo algorithm (rjMCMC, Green 1995) coupled with the Parallel Tempering technique (Dettmer \& Dosso 2012; Sambridge 2014). The utilized parametrization allows us to treat the number of layers as an unknown parameter of the inverse problem (Bodin et al. 2012). In particular, we treat the number of layers as a measure of complexity of the model space using an explicit Occam's razor following approach by Hallo \& Gallovič (2020). Moreover, the Bayesian formulation (e.g. Jaynes 2003) allow us to include additional prior assumptions on the model space using a prior probability. To this end, we utilize a novel multizonal formulation of the prior probability density function (prior PDF) of model parameters (i.e. $S$ - and $P$-wave velocities, mass density, and additionally Poisson's ratio). The multizonal formulation defines multiple horizontal subsurface zones with specific, yet efficiently homogeneous, prior PDF of model parameters. Such a formulation allows us to consider site-specific assumptions on the model space coming from, for example stratigraphic logs, standard penetration tests, known groundwater level and bedrock depth, and improve the efficiency of the model space exploration. Finally, our inversion approach is validated based on synthetic tests and applied to real surface wave data.

\section{METHOD}

Here we describe the Multizonal Transdimensional Inversion (MTI) approach. It consists of four main keystones: (1) appropriate model parametrization, (2) joint data misfit in standardized units, (3) multizonal yet efficiently homogeneous prior and (4) self-adapting exploration of the transdimensional model space.

\subsection{Model parametrization}

We assume a subsurface model composed of horizontal and isotropic layers defined by $S$ - and $P$-wave velocities ( $v_{\mathrm{S}}$ and $v_{\mathrm{P}}$, respectively) and mass density $(\rho)$. This model is parametrized following Bodin et al. (2012) using multiple Voronoi nuclei (VNi), where inter-layer boundaries (i.e. interfaces) are defined as equidistant between two adjacent nuclei at depth. Hence, an interface is parametrized by the depth of two adjacent nuclei. As the absolute resolving power of the surface wave data decreases with depth, we parametrize positions of VNi along the profile by taking their natural logarithm, thus providing higher resolution on the shallower part. The logarithmic depth (ln-depth, $h$ ) is limited by a minimum threshold value (i.e. $h^{\mathrm{min}}=\ln (\operatorname{dept} h[\mathrm{~m}]>0)$ ), representing the uppermost considered depth of the model space. The total number of $\mathrm{VNi}(N)$ is allowed to be variable, as well as their position in the ln-depth domain. Note that we do not constrain the layer thickness by any minimum value. Model parameters (i.e. $h, v_{\mathrm{S}}, v_{\mathrm{P}}$ and $\rho$ ) assigned to VNi are stored along the rows of matrix $\boldsymbol{\Phi}$, whose size is $N \times 4$. To comply with the multizonal prior on model space, we formally divide the matrix $\boldsymbol{\Phi}$ into $Z$ submatrices $\boldsymbol{\Lambda}_{i}(i \in\{1, \ldots, Z\})$ representing horizontal zones as

$\boldsymbol{\Phi}=\left[\begin{array}{c}\boldsymbol{\Lambda}_{1} \\ \vdots \\ \boldsymbol{\Lambda}_{Z}\end{array}\right] \equiv\left[\begin{array}{c}\boldsymbol{\Lambda}_{\text {soil }} \\ \vdots \\ \boldsymbol{\Lambda}_{\text {bedrock }}\end{array}\right]$

To each submatrix $\boldsymbol{\Lambda}_{i}$ is assigned a specific (but variable) number of VNi $\lambda_{i}$, with a minimum of one nucleus per zone. Then, the submatrix $\boldsymbol{\Lambda}_{i}$ of zone $i$ consists of four single-column vectors $\left(\mathbf{h}_{i}, \mathbf{v}_{\mathrm{S} i}, \mathbf{v}_{\mathrm{P} i}\right.$, and $\left.\boldsymbol{\rho}_{i}\right)$ as follows:

$\boldsymbol{\Lambda}_{i}=\left[\begin{array}{cccc}h_{i}^{(1)} & v_{\mathrm{S} i}^{(1)} & v_{\mathrm{P} i}^{(1)} & \rho_{i}^{(1)} \\ \vdots & \vdots & \vdots & \vdots \\ h_{i}^{\left(\lambda_{i}\right)} & v_{\mathrm{S} i}^{\left(\lambda_{i}\right)} & v_{\mathrm{P} i}^{\left(\lambda_{i}\right)} & \rho_{i}^{\left(\lambda_{i}\right)}\end{array}\right]$

where the total number of VNi in matrix $\boldsymbol{\Phi}$ is $N=\sum_{i=1}^{Z} \lambda_{i}$. Logically, we enforce that VNi in the lower zones must be deeper than VNi in the upper zones, that is $\forall i \in\{1, \ldots, Z-1\}, \max \left(\mathbf{h}_{i}\right)<\min \left(\mathbf{h}_{i+1}\right)$. The 1-D layered model of the subsurface is then fully parametrized by the matrix $\boldsymbol{\Phi}$.

Note that zone and layer thicknesses are not related; hence, an interzone boundary ( $z$-interface) must not be interpreted as an interface of the layered velocity model. To comply with the model space definition, the model given in eq. (1) is expressed as a vector

$\mathbf{w}_{N}=\operatorname{vec}(\boldsymbol{\Phi})$,

where $\operatorname{vec}(\cdot)$ denotes vectorization of a matrix, and index $N$ is proportional to the resultant vector length. 


\subsection{The forward problem}

Exploring the model space requires solving the forward problem multiple times, and hence, it should be fast and efficient. In this paper, we use the Geopsy software package (Wathelet et al. 2020) for the forward modelling, which relies on the Thomson-Haskell matrix method (Thomson 1950; Haskell 1953) modified by Dunkin (1965). This numerical package can compute dispersion curves associated with the fundamental and higher modes of Rayleigh and Love waves and the ellipticity curve of the fundamental mode of Rayleigh waves in a given layered medium. Alternatively, Geopsy can also produce the signed Rayleigh wave ellipticity, which can be used to compute the ellipticity angle (Maranò et al. 2017). The solution of the forward problem for the model vector $\mathbf{w}_{N}$ in eq. (3) can be expressed using a forward operator $\mathbf{g}(\cdot)$ as

$\mathbf{d}=\mathbf{g}\left(\mathbf{w}_{N}\right)$,

where vector $\mathbf{d}$ contains all the modelled data (i.e. modelled dispersion curves and ellipticity or ellipticity angle). We would like to emphasize that our method is not bounded to this particular forward solver, and in principle, any other suitable algorithm can be used. The described procedure can also be applied to the inversion of Stoneley or Scholte waves (Shynkarenko et al. 2021).

\subsection{Transdimensional Bayesian inference}

Following Tarantola (2005), we introduce an abstract $n$-dimensional vector space $\mathcal{R}^{n}$, where each vector $\mathbf{w}\left(\mathbf{w} \in \mathcal{R}^{n}\right)$ represents a model of the system. In our case, the length of the model vector $\mathbf{w}$ (and the dimension $n$ of the model space) varies, as we use a varying number of Voronoi nuclei $N$. It leads to the transdimensional formulation of Green $(1995,2003)$. We aim to explore transdimensional models $\mathbf{m}=\left(k, \mathbf{w}_{k}\right)$, where $k$ is indexing the state of the model space (in our case $k \equiv N$ and $n=4 N$ ). Following Green (2003), the solution of the transdimensional Bayesian inversion of the observed data $\mathbf{d}_{\mathrm{obs}}$ is given by the posterior conditional PDF on mode space, that is $p\left(\mathbf{m} \mid \mathbf{d}_{\mathrm{obs}}\right)$, as

$p\left(\mathbf{m} \mid \mathbf{d}_{\mathrm{obs}}\right)=\frac{1}{c} p(k) p\left(\mathbf{w}_{k} \mid k\right) p\left(\mathbf{d}_{\mathrm{obs}} \mid k, \mathbf{w}_{k}\right)$,

where $c$ is a normalization constant, $p(k)$ is a prior probability of the model state $k$ (i.e. the expected total number of $\mathrm{VNi}$ ) and $p\left(\mathbf{w}_{k} \mid k\right)$ is a prior conditional PDF of model parameters given state $k$. The term $p\left(\mathbf{d}_{\text {obs }} \mid k, \mathbf{w}_{k}\right)$ is the conditional PDF of data given model $\left(k, \mathbf{w}_{k}\right)$, that is $p\left(\mathbf{d}_{\mathrm{obs}} \mid k, \mathbf{w}_{k}\right)=p\left(\mathbf{d}_{\mathrm{obs}} \mid \mathbf{m}\right)$, which is the so-called likelihood function. For details on the transdimensional inference (see, e.g. Malinverno 2002; Sambridge et al. 2006; Bodin \& Sambridge 2009; Gallagher et al. 2009; Dettmer et al. 2010; Hallo \& Gallovič 2020).

\subsection{The likelihood function}

The likelihood function $p\left(\mathbf{d}_{\text {obs }} \mid \mathbf{m}\right)$ in eq. (5) quantifies how well model $\mathbf{m}$ (where $\mathbf{m}=\left(k, \mathbf{w}_{k}\right)$ ) fits the observed data $\mathbf{d}_{\text {obs }}$ based on modelled data from eq. (4). When assuming Gaussian data errors, this function can be recast in a matrix form (e.g. Tarantola 2005, pp. 62-64) given by the proportionality

$p\left(\mathbf{d}_{\mathrm{obs}} \mid \mathbf{m}\right) \propto \exp \left(-\frac{1}{2}\left(\mathbf{R d}_{\mathrm{obs}}-\mathbf{R g}(\mathbf{m})\right)^{\mathrm{T}}\left(\mathbf{R} \mathbf{d}_{\mathrm{obs}}-\mathbf{R g}(\mathbf{m})\right)\right)$,

where $\mathbf{R} \mathbf{d}_{\mathrm{obs}}$ and $\mathbf{R} \mathbf{g}(\mathbf{m})$ are so-called standardized observed and modelled data, respectively. $\mathbf{R}$ is an upper triangular matrix resulting from the Cholesky decomposition of the inverse data covariance matrix $\mathbf{C}$, that is $\mathbf{C}^{-1}=\mathbf{R}^{\mathrm{T}} \mathbf{R}$. Matrix $\mathbf{C}$ characterizes errors of both observed data (the uncertainty of measured dispersion and ellipticity curves) and modelled data (the uncertainty due to approximating the Earth's crust as a 1-D layered model). Note that the likelihood function in eq. (6) evaluates the misfit of residuals in joint standardized units of data (i.e. the data weighted by the inverse of the square root of their covariance). Then, the term joint inversion indicates the use of mixed physical quantities in the data space (subvectors of $\mathbf{d}_{\mathrm{obs}}$ ).

The full covariance matrix $\mathbf{C}$ of dispersion and ellipticity curves is generally unknown, although there has been growing interest in their estimation recently (e.g. Lai et al. 2005). As an alternative, we may use either an unknown scaling factor of these matrices (i.e. hierarchical Bayes; Bodin et al. 2012) or an assumed data variance instead of full covariance matrices. We opt for the second option: approximate the data covariance matrix as its diagonal, that is $\mathbf{C}=\sigma^{2} \mathbf{I}$, where $\mathbf{I}$ is the identity matrix, and $\sigma$ is a vector of standard deviation of data errors (having Gaussian distribution). Then $\mathbf{R}=\sigma^{-1} \mathbf{I}$, and the likelihood function in eq. (6) further simplifies to (e.g. Dettmer et al. 2007)

$p\left(\mathbf{d}_{\mathrm{obs}} \mid \mathbf{m}\right) \propto \exp \left(-\frac{1}{2} \sum_{l=1}^{L}\left(\frac{d_{\mathrm{obs} l}}{\sigma_{l}}-\frac{g_{l}(\mathbf{m})}{\sigma_{l}}\right)^{2}\right)$,

where $L$ is the total number of observed data discrete samples (indexed by $l$ ). Eq. (7) is the likelihood function implemented in our approach; nevertheless, if the covariance matrix is known, the method may be enhanced using eq. (6).

The data misfit within the likelihood function has merely a statistical meaning, as it expresses the sum-of-squares of residuals of standardized data (i.e. the sum of squared residuals divided by the data variance). We can therefore define the goodness of the fit of the model 
(a)

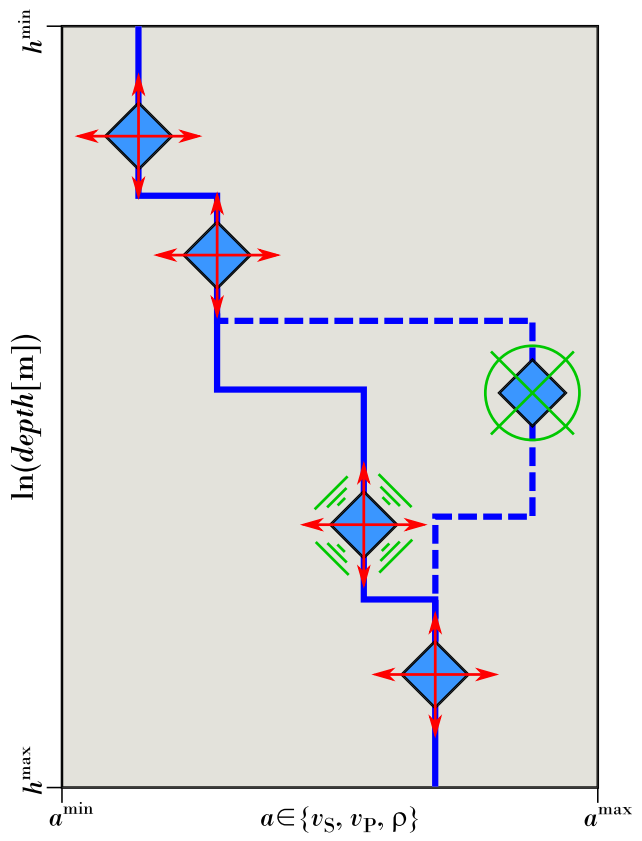

(b)

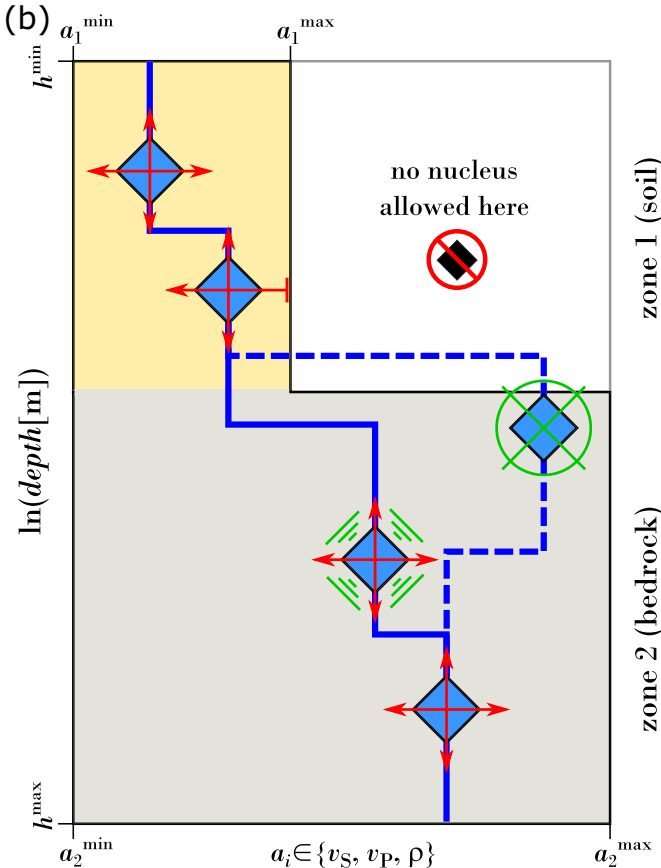

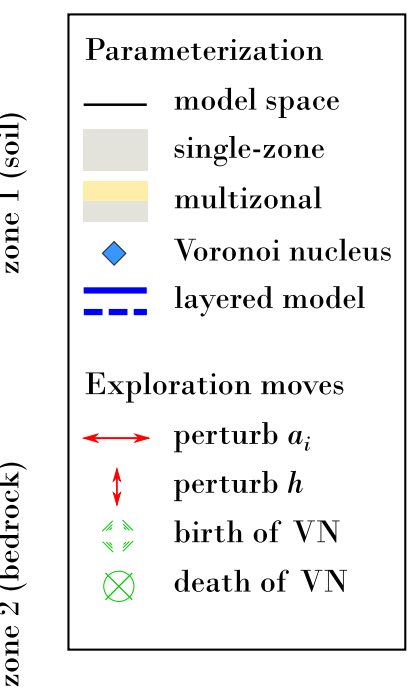

Figure 1. Layered model parametrization by variable number of Voronoi nuclei with (a) single-zone (b) multizonal prior PDF of model parameters. Layered models (solid and dashed blue lines) are explored using random moves in the model space (red and green signs).

$\mathbf{m}$ in terms of data variance reduction $\phi_{\mathrm{VR}}$ as follows:

$\phi_{\mathrm{VR}}(\mathbf{m}) \stackrel{\underline{\text { def }}}{=}\left(1-\frac{1}{L} \sum_{l=1}^{L} \frac{\left(d_{\mathrm{obs} l}-g_{l}(\mathbf{m})\right)^{2}}{\sigma_{l}^{2}}\right) \times 100$ per cent.

Note that this definition of the data variance reduction differs from other common definitions in seismic waveform inversions (e.g. Ford et al. 2009; Sokos \& Zahradník 2013; Hallo et al. 2017) and from the classic variance reduction of Monte Carlo methods. The data variance reduction in eq. (8) reaches a maximum value of 100 per cent for a perfect data fit with zero residuals, 0 per cent for residuals of $\sigma_{l}$ and -300 per cent for $2 \sigma_{l}$ of data errors on average. We qualitatively define the data fit as 'perfect', 'good' and 'fair' for residuals $<0.25 \sigma_{l},<0.5 \sigma_{l}$ and $<0.75 \sigma_{l}$ of data errors on average, respectively.

\subsection{The multizonal prior}

The Bayesian logic (Bayes 1763) allows us to include additional prior assumptions on the model space by using the prior probability of model parameters (e.g. Jaynes 2003). At the same time, we would like to be conservative and avoid biased solutions caused by too complicated priors (which may occur even in non-Bayesian inversions through an inappropriate parametrization). We therefore use a multizonal formulation of the prior PDF of model parameters (see Fig. 1).

The multizonal formulation (see eqs 1 and 2) defines a countable number $Z$ of independent horizontal zones. These do not overlap and are contiguous without gaps at depth (Fig. 1b). Each $i$ th zone has assigned a variable number of Voronoi nuclei $\lambda_{i}$ with model parameters stored in vectors $\mathbf{h}_{i}, \mathbf{v}_{\mathrm{S} i}, \mathbf{v}_{\mathrm{P} i}$ and $\boldsymbol{\rho}_{i}$. The VNi count (i.e. the length of these vectors) is variable as VNi are allowed to change zone as well as we use the transdimensional formulation (i.e. a variable number of VNi in total). The prior PDF from eq. (5) is then expressed as

$p(k) p\left(\mathbf{w}_{k} \mid k\right)=p(k) \prod_{i=1}^{Z}\left(p\left(\mathbf{h}_{i}\right) p\left(\mathbf{v}_{\mathrm{S} i}, \mathbf{v}_{\mathrm{P} i}, \boldsymbol{\rho}_{i} \mid i\right)\right)$,

where $p\left(\mathbf{v}_{\mathrm{S} i}, \mathbf{v}_{\mathrm{P} i}, \boldsymbol{\rho}_{i} \mid i\right)$ is a conditional prior PDF of model parameters given zone $i$, and $p\left(\mathbf{h}_{i}\right)$ is prior PDF of ln-depths of $\lambda_{i} \mathrm{VNi}$ for the $i$ th zone (the prior PDF of ln-depth is zone-independent). The conditional prior PDF in eq. (9) can be separated into three prior PDFs for each specific model parameter (assumed independent from each other) as follows:

$p\left(\mathbf{v}_{\mathrm{S} i}, \mathbf{v}_{\mathrm{P} i}, \boldsymbol{\rho}_{i} \mid i\right)=p\left(\mathbf{v}_{\mathrm{S} i} \mid i\right) p\left(\mathbf{v}_{\mathrm{P} i} \mid i\right) p\left(\boldsymbol{\rho}_{i} \mid i\right)$.

The three terms in the right-hand side of eq. (10) are conditional prior PDFs of $v_{\mathrm{S}}, v_{\mathrm{P}}$ and $\rho$ given zone $i$. In other words, zone-specific prior PDFs can be set to these three properties. In our approach, the prior PDF of a single parameter $a$ (the substitution $a \in\left\{v_{\mathrm{S}}, v_{\mathrm{P}}, \rho\right\}$ applies) 
is a constant defined on an interval of plausible values $A_{i}=\left\{a_{i} \in R \mid a_{i}^{\min } \leq a_{i} \leq a_{i}^{\max }\right\}$ for each zone $i$ :

$p\left(a_{i} \mid i\right)=\left\{\begin{array}{cl}\frac{1}{a_{i}^{\max }-a_{i}^{\min }} & \text { if } a_{i} \in A_{i} \\ 0 & \text { otherwise. }\end{array}\right.$

The zone-specific prior PDF is then efficiently homogeneous (i.e. weakly informative prior), or, in other words, uniform within the interval of plausible values $A_{i}$ (e.g. Bodin et al. 2012; Hallo \& Gallovič 2020). For the prior PDF of the ln-depth of single VN, that is $p(h)$, we assume a constant value on the whole interval $B=\left\{h \in R \mid h^{\min } \leq h \leq h^{\text {max }}\right\}$ leading, also in this case, to an efficiently homogeneous prior PDF.

To summarize, eq. (9) defines the multizonal conditional prior PDF, and eq. (11) shows the efficiently homogeneous prior PDF of model parameters $v_{\mathrm{S}}, v_{\mathrm{P}}$ and $\rho$. It allows us to use zone-specific minimum and maximum plausible values $v_{\mathrm{S} i}^{\min }, v_{\mathrm{S} i}^{\max }, v_{\mathrm{P} i}^{\min }, v_{\mathrm{P} i}^{\max }, \rho_{i}^{\min }$ and $\rho_{i}^{\max }$, where $i \in\{1, \ldots, Z\}$. Note that this formulation does not bound the prior PDF to a particular layer (i.e. VN), yet it makes it dependent on the profile depth (as suggested by index $i$ ).

\subsection{Exploration of the transdimensional model space}

The posterior PDF of model parameters $p\left(\mathbf{m} \mid \mathbf{d}_{\text {obs }}\right)$ in eq. (5) is the target of the inverse problem. Since it cannot be evaluated analytically due to non-linearity, it must be explored by a sampling algorithm (e.g. Gelman et al. 2013; Supplement S1). For this purpose, we utilize the reversible jump Markov chain Monte Carlo algorithm (rjMCMC, Green 1995). The rjMCMC is based on the Metropolis-Hastings algorithm (M-H algorithm; Metropolis et al. 1953; Hastings 1970), which samples the posterior PDF by a series of random steps, that is $\mathbf{m}_{0} \stackrel{\alpha\left(\mathbf{m}^{\prime} \mid \mathbf{m}_{0}\right)}{\rightarrow} \mathbf{m}_{1} \stackrel{\alpha\left(\mathbf{m}^{\prime} \mid \mathbf{m}_{1}\right)}{\rightarrow} \mathbf{m}_{2} \rightarrow \ldots$. For details on the transdimensional model space sampling (i.e. the across-model simulations within the union state space) based on rjMCMC (see, e.g. Green 1995, 2003; Sambridge et al. 2006; Dettmer et al. 2010; Hallo \& Gallovič 2020).

The workflow of the rjMCMC algorithm utilized in our MTI approach is summarized as follows (points I-VIII):

(I) Create an initial model $\mathbf{m}_{0}$. This model is drawn randomly from the prior PDF.

(II) Randomly selects the move type. There are three types of random moves in the rjMCMC: 'perturb move' (randomly perturb the existing model), 'birth move' (add one arbitrarily VN) and 'death move' (remove one arbitrarily VN). We use an equal probability of the birth and death move proposals (not to be confused with the prior probability).

(III) Propose a new model $\mathbf{m}^{\prime}$ from the current model $\mathbf{m}$ by using the selected move type (IIIa-IIId):

(IIIa) Perturb move - All parameters of the current model $\mathbf{m}$ are perturbed using Gaussian proposal distribution independently from each other. The proposal distribution is recentred at each step of the Markov chain to the current value (i.e. the Gaussian random walk, see Supplement S2). To preserve both the homogeneous prior and the efficiency of the algorithm, we utilize the first-order mirroring of the proposal distribution at the boundaries $a_{i}^{\min }$ and $a_{i}^{\max }$ following the approach of Hallo \& Gallovič (2020).

(IIIb) Interzonal move - This move (sub-type of perturb move) can only be triggered by the performed perturb move if any of the VNi within the proposed model $\mathbf{m}^{\prime}$ crosses a $z$-interface (through perturbation of $h$ by Gaussian random walker at ln-depth). If this occurs, check if the move is possible and transfer the VN between submatrices $\boldsymbol{\Lambda}_{i}$ and $\boldsymbol{\Lambda}_{i+1}$, (see eq. 2).

(IIIc) Birth move - The proposed model $\mathbf{m}^{\prime}$ is obtained by adding one VN to the current model $\mathbf{m}$. Four model parameters for the new $\mathrm{VN}$ (i.e. $h, v_{\mathrm{S}}, v_{\mathrm{P}}$ and $\rho$ ) are drawn randomly from the prior PDF (i.e. so-called birth from prior). Note that the proposed model vector $\mathbf{m}^{\prime}$ expands vector $\mathbf{m}$ with four new dimensions.

(IIId) Death move - Removing one arbitrary VN from model $\mathbf{m}$ produces a new proposed model $\mathbf{m}^{\prime}$, resulting in a collapse of four dimensions.

(IV) Compute the forward problem in eq. (4) and evaluate the likelihood function $p\left(\mathbf{d}_{\mathrm{obs}} \mid \mathbf{m}\right)$ defined in eq. (7) on both current $\mathbf{m}$ and proposed $\mathbf{m}^{\prime}$.

(V) The M-H algorithm rejects or accepts the proposed model $\mathbf{m}^{\prime}$ based on the acceptance probability $\alpha\left(\mathbf{m}^{\prime} \mid \mathbf{m}\right)$ (Metropolis et al. 1953; Hastings 1970). Following Hallo \& Gallovič (2020), we use M-H acceptance probabilities with prescribed reciprocal distribution $p(k) \propto k^{-1}$ for the prior probability of the model states $p(k)$; for details about the rjMCMC with the reciprocal prior see Supplement S3. Such prior works as an explicit Occam's razor, where a better data fit is required to increase the number of VNi in the accepted models in the long-term average (i.e. the self-adapting complexity of models). This is a law of parsimony where the layered profile samples are as simple as possible but not simpler than required by the observed data (see Appendix B). To increase the efficiency of the rjMCMC, we apply the Parallel Tempering technique following approach by Sambridge (2014), where the sampling is performed by multiple Markov chains (cold sampling chains and hot exploration chains) that may swap their assigned models. Following Hallo \& Gallovič (2020) and as shown in Appendix A, the $\mathrm{M}-\mathrm{H}$ acceptance probabilities for perturb $\alpha_{\mathrm{P}}$, birth $\alpha_{\mathrm{B}}$, and death $\alpha_{\mathrm{D}}$ moves are as follows:

$\alpha_{\mathrm{P}}\left(\mathbf{m}^{\prime} \mid \mathbf{m}, \gamma\right)=\min \left(1,\left(\frac{p\left(\mathbf{d}_{\mathrm{obs}} \mid \mathbf{m}^{\prime}\right)}{p\left(\mathbf{d}_{\mathrm{obs}} \mid \mathbf{m}\right)}\right)^{1 / \gamma}\right)$, 
$\alpha_{\mathrm{B}}\left(\mathbf{m}^{\prime} \mid \mathbf{m}, \gamma\right)=\min \left(1, \frac{k}{k+1}\left(\frac{p\left(\mathbf{d}_{\mathrm{obs}} \mid \mathbf{m}^{\prime}\right)}{p\left(\mathbf{d}_{\mathrm{obs}} \mid \mathbf{m}\right)}\right)^{1 / \gamma}\right)$,

$\alpha_{\mathrm{D}}\left(\mathbf{m}^{\prime} \mid \mathbf{m}, \gamma\right)=\min \left(1, \frac{k}{k-1}\left(\frac{p\left(\mathbf{d}_{\mathrm{obs}} \mid \mathbf{m}^{\prime}\right)}{p\left(\mathbf{d}_{\mathrm{obs}} \mid \mathbf{m}\right)}\right)^{1 / \gamma}\right)$,

where $\gamma \geq 1$ is an additional parameter of the Parallel Tempering called temperature (Geyer 1991; Sambridge 2014). Note that terms $k /(k+1)$ and $k /(k-1)$ in eqs (13) and (14) originate from the explicit prior probability of the model space states $p(k)$. Also note that a comparison with an inversion without these additional terms is shown in Appendix B. The M-H acceptance probability for the interzonal move $\alpha_{\mathrm{Z}}$ is given by (see Appendix A)

$\alpha_{\mathrm{Z}}\left(\mathbf{m}^{\prime} \mid \mathbf{m}, j, j^{\prime}, \gamma\right)=\min \left(1, \frac{p\left(v_{\mathrm{S}_{j}} \mid j^{\prime}\right) p\left(v_{\mathrm{P} j} \mid j^{\prime}\right) p\left(\rho_{j} \mid j^{\prime}\right)}{p\left(v_{\mathrm{S} j} \mid j\right) p\left(v_{\mathrm{P} j} \mid j\right) p\left(\rho_{j} \mid j\right)}\left(\frac{p\left(\mathbf{d}_{\mathrm{obb}} \mid \mathbf{m}^{\prime}\right)}{p\left(\mathbf{d}_{\mathrm{obs}} \mid \mathbf{m}\right)}\right)^{1 / \gamma}\right)$.

In the interzonal move, one $\mathrm{VN}$ is transferred from the source zone $j$ to the target zone $j^{\prime}\left(\left\{j, j^{\prime}\right\} \subseteq\{1, \ldots, Z\}\right)$ while its properties $v_{\mathrm{S} j}, v_{\mathrm{P} j}$ and $\rho_{j}$ remain unchanged. The conditional prior PDFs in eq. (15) can be evaluated for a given pair of zones by means of eq. (11). Finally, the M-H algorithm rejects or accepts the proposed model based on the acceptance probabilities defined in eqs (12)-(15). An analytical derivation of the $\mathrm{M}-\mathrm{H}$ acceptance probabilities for the transdimensional model space with the multizonal prior is shown in Appendix A.

(VI) The current Markov chain tries to exchange the temperature $\gamma$ with another chain (see, e.g. Sambridge 2014; Valentová et al. 2017).

(VII) The model of the Markov chain with $\gamma=1$ (sampling chain) is saved into the ensemble of solutions. This action only occurs if the chain performed a sufficient (user-defined) number of burn-in steps, after which the random walk is assumed to be stationary. The convergence to this state is estimated by continuously monitoring the rate of change of the data variance reduction (see Supplement S1).

(VIII) The whole procedure is repeated from point II to VII until (a) the maximum (user-defined) number of Markov chain steps is reached or (b) terminated by the user. Both options are viable as the model samples are saved continuously on the hard drive.

\section{IMPLEMENTATION DETAILS}

The developed MTI algorithm was implemented in a numerical package written in Fortran90. The code utilizes the Parallel Tempering library of Sambridge (2014) and some routines from the Parametric Slip Inversion numerical package of Hallo \& Gallovič (2020). The observed data vector $\left(\mathbf{d}_{\mathrm{obs}}\right)$ consists of discrete values of dispersion curves in slowness units $\left[\mathrm{sm}^{-1}\right]$, common logarithm of the Rayleigh wave ellipticity $\left[\log _{10}\left(\operatorname{abs}\left(\mathbf{d}_{\text {ell }}\right)\right)\right]$, or the ellipticity angle $\left[\tan ^{-1}\left(\mathbf{d}_{\mathrm{ell}}\right)\right]$. These data are supplemented with uncertainties by means of assumed $1 \sigma$ of data errors having Gaussian distribution. The output of numerical routines is an ensemble of layered velocity models saved on the hard drive. Such an ensemble of models is used for evaluating marginal histograms of subsurface properties of interest.

\subsection{The apparent effective prior}

Model parameters $v_{\mathrm{S}}, v_{\mathrm{P}}$ and $\rho$ are treated as independent from each other in eq. (10). Nevertheless, additional physics-based conditions on interparameter combinations can be imposed within a computational code when inverting for the Earth's subsurface. These conditions apparently influence the prescribed prior PDFs of model parameters, giving origin to the so-called effective prior (not to be confused with the efficiently homogeneous prior nor the prior PDF). This effective prior is influenced by the prior PDF, behaviour of the deployed random walk algorithm, and additional conditions in the computational code. The prior PDF is expressed analytically, the behaviour of the random walk algorithm is investigated in Supplements S2 and S3, and hence the last remaining is the effect of additional physics-based conditions.

To the first possible additional condition, the dependency of doublets $v_{\mathrm{S} i}$ and $v_{\mathrm{P} i}$ within each VN can be expressed in terms of plausible values of Poisson's ratio $(\eta)$ for rock and soil materials. It is preferable to preserve the prior of $S$-wave velocity (which is the main parameter) and assume eq. (10) in the form of

$p\left(\mathbf{v}_{\mathrm{S} i}, \mathbf{v}_{\mathrm{P} i}, \boldsymbol{\rho}_{i} \mid i\right)=p\left(\mathbf{v}_{\mathrm{S} i} \mid i\right) p\left(\mathbf{v}_{\mathrm{P} i} \mid \mathbf{v}_{\mathrm{S} i}, i\right) p\left(\boldsymbol{\rho}_{i} \mid i\right)$,

where $p\left(\mathbf{v}_{\mathrm{P} i} \mid \mathbf{v}_{\mathrm{Si}}, i\right)$ is prior PDF of $P$-wave velocities conditioned by $S$-wave velocities and implicitly also by minimum/maximum plausible values of the Poisson's ratio given zone $i$. Eq. (16) shows that we can include a condition on the Poisson's ratio within a computational code that does not interfere with the prior PDF of $S$-wave velocity. In particular, the condition rejecting proposed models outside the interval of plausible Poisson's ratios $\left[\eta_{i}^{\min }, \eta_{i}^{\max }\right]$ take an effect when perturbing $P$-wave velocity.

As the second additional condition, we may impose a requirement on the presence of a positive velocity gradient. Velocities of all VNi (i.e. elements of vectors $\mathbf{v}_{\mathrm{S} i}$ and $\mathbf{v}_{\mathrm{P} i}$ ) are treated as independent from each other in eq. (11). It means that low-velocity zones are permitted to 
(a)

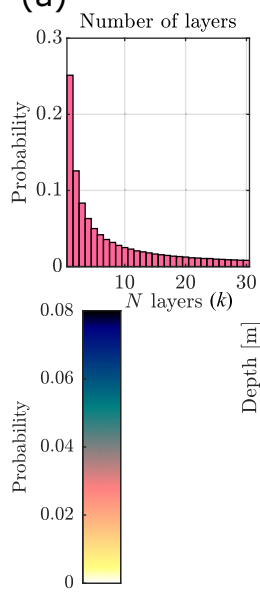

(e)

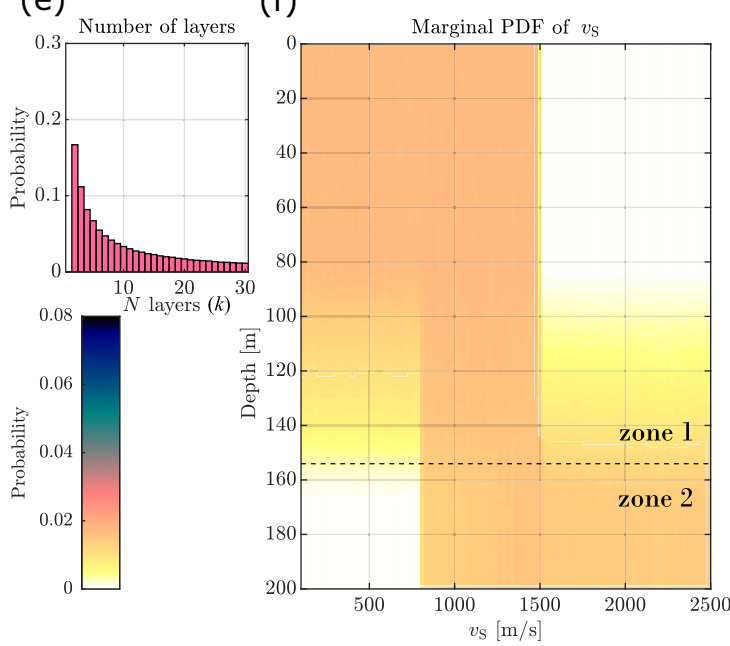

(b)

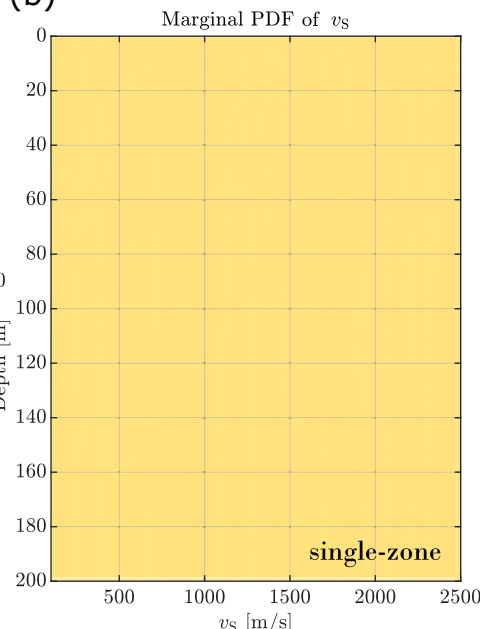

(f) (c)

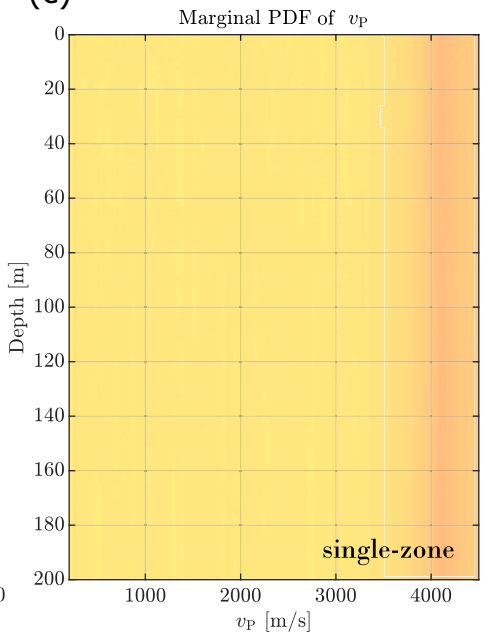

(g)

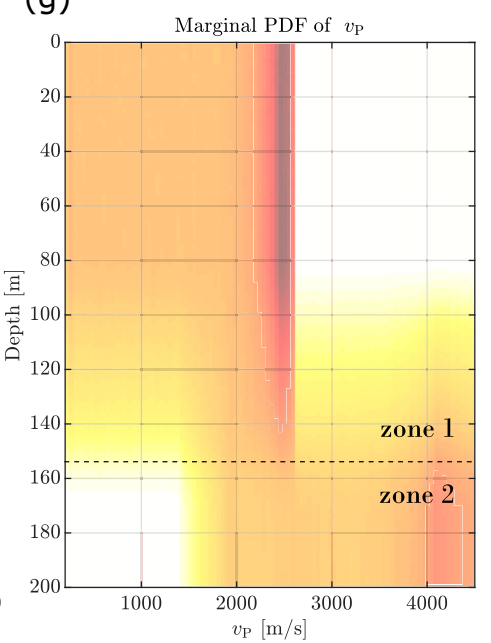

(d)

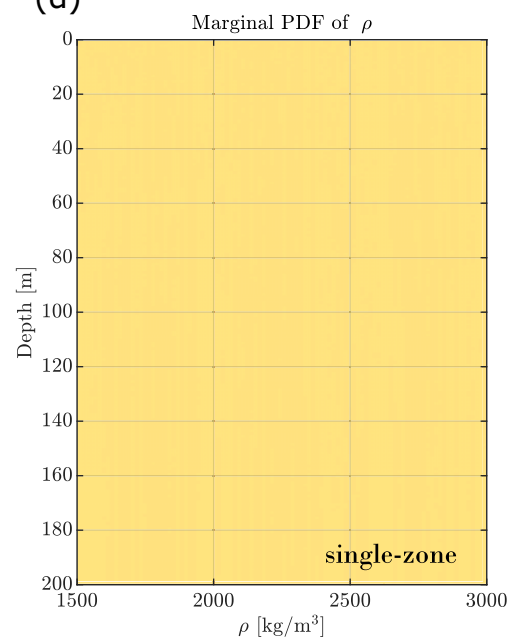

(h)

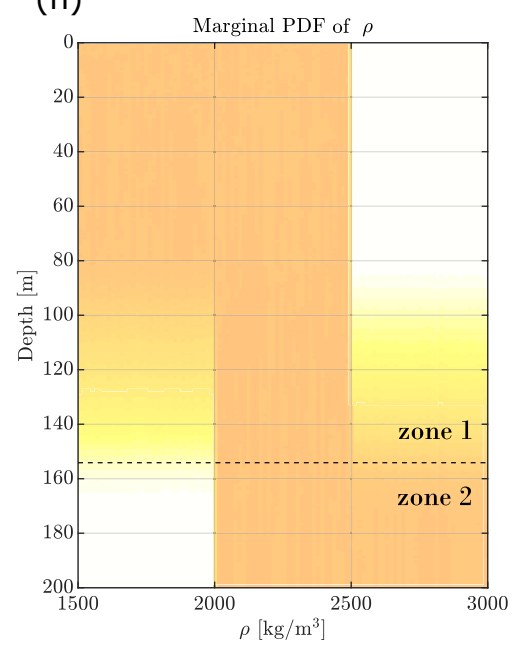

Figure 2. The apparent effective prior in the test with allowed low-velocity zones (default code settings). Upper panels show the effective prior in the single-zone model space; bottom panels show the effective prior in the multizonal model space. Panels (a) and (e) show histograms for effective prior probability of the number of layers. The effective marginal prior PDFs of $S$-wave velocity in panels (b) and (f), $P$-wave velocity in panels (c) and (g) and mass density in panels (d) and (h) are shown by colourscale.

occur freely in the whole profile by default. However, in justified cases, it is reasonable to avoid low-velocity zones by forbidding interfaces delimiting velocity inversion (IVIs). In particular, we define a threshold $h^{\text {thr }}$ expressed in ln-depth, representing the maximum permitted depth of an IVI. If $h^{\text {thr }}=h^{\max }$, the low-velocity zones are permitted to occur freely in the whole profile. On the other hand, $h^{\text {thr }}=h^{\text {min }}$ settings is the constraint requiring a positive velocity gradient in the whole profile. Such a constraint of inversion may be required in some specific cases due to the non-uniqueness of this inverse problem (e.g. Wathelet 2008). We would like to emphasize that both of these additional conditions can be always (de)activated in the computational code settings.

The analytical prescription of the effective prior on the model space would be rather complex; nevertheless, its knowledge may be beneficial for interpretation purposes (e.g. identifying features having origin in prior). For this purpose, we imaged the effective prior by a random exploration of the model space with the likelihood function set to a constant (i.e. $p\left(\mathbf{d}_{\mathrm{obs}} \mid \mathbf{m}\right)=1$ ). This can be achieved by a dry-run test of MTI computational program that, at the same time, validates the correctness of the algorithm implementation. Expectations on such a test are: (a) imaging of the effective prior and (b) validation of the internal balance in the $\mathrm{M}-\mathrm{H}$ algorithm implementation. We show results of dry-run tests for the single-zone and multizonal transdimensional model space with different activated conditions in Figs 2 and 3. The MTI computational program produced ensembles of models drawn from the effective prior based on 1000 Markov chains of Parallel Tempering (990 exploration and 10 sampling chains). For each of these chains, the length of burn-in and production phases were set to 10000 and $\sim 100000$ steps, respectively. Hence, the program was able to test $\sim 110000000$ models, and it produced $\sim 1000000$ random samples. The resultant ensemble of random samples is used to construct 2-D marginal distributions. This is a common way to visualize the model space; however, such a distribution do not show the full effect nor correlations of the high-dimensional space.

The apparent effective prior in the model space with permitted low-velocity zones is shown in Fig. $2\left(h^{\text {thr }}=h^{\text {max }}\right.$ settings of the computational code). The upper and bottom panels refer to tests with the single-zone and multizonal model space, respectively. The effective 
(a)

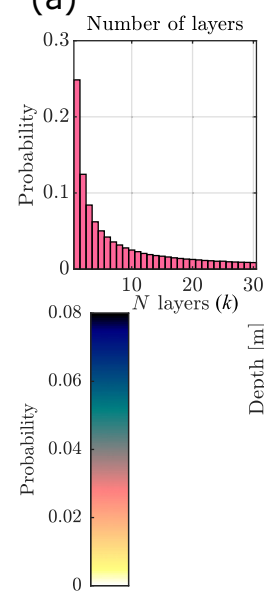

(e)

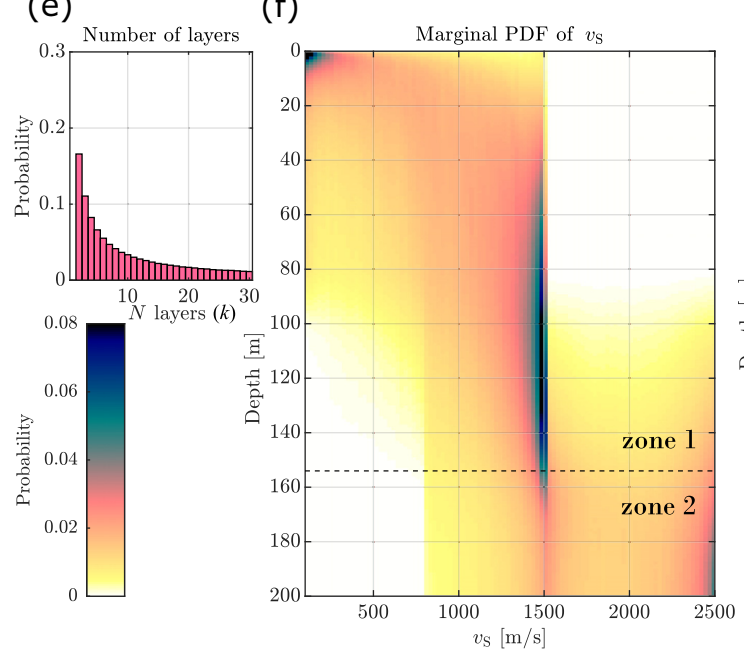

(c)

(b)

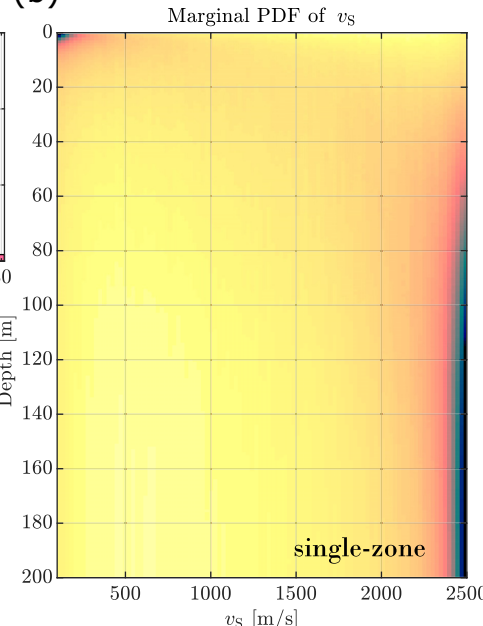

(f)

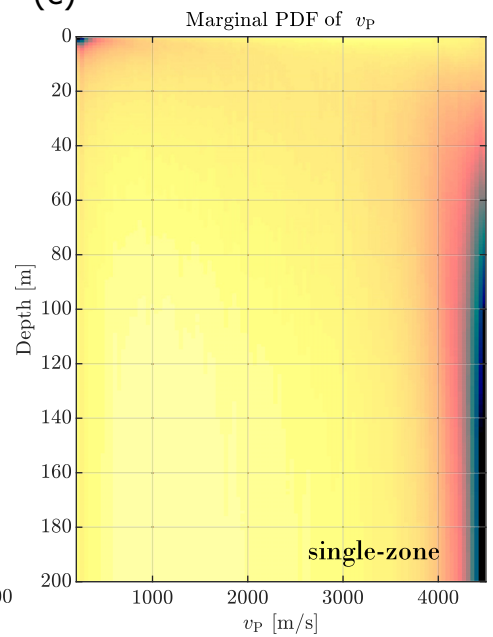

(g)

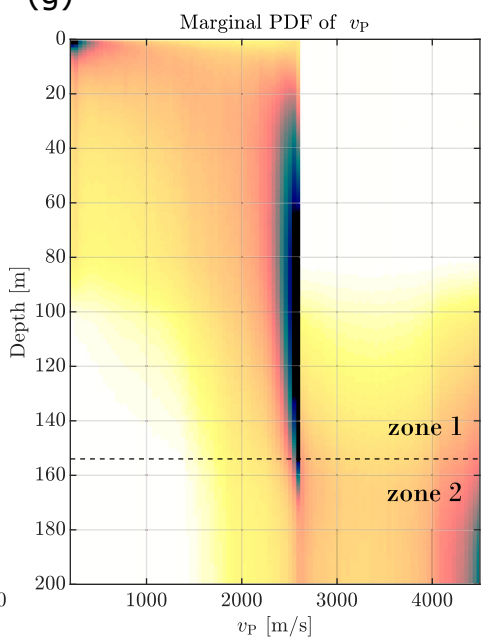

(d)

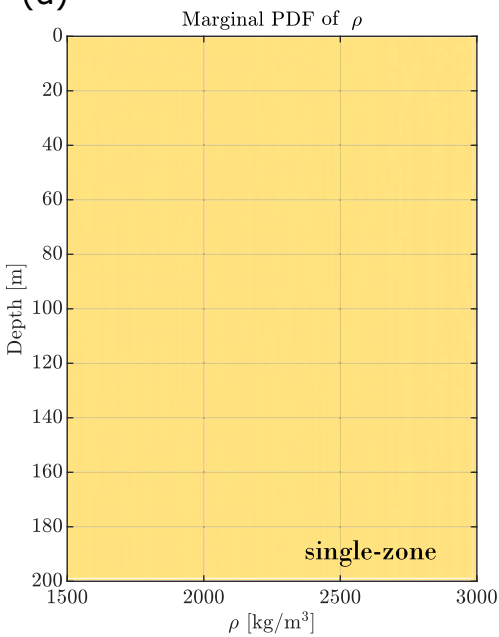

(h)

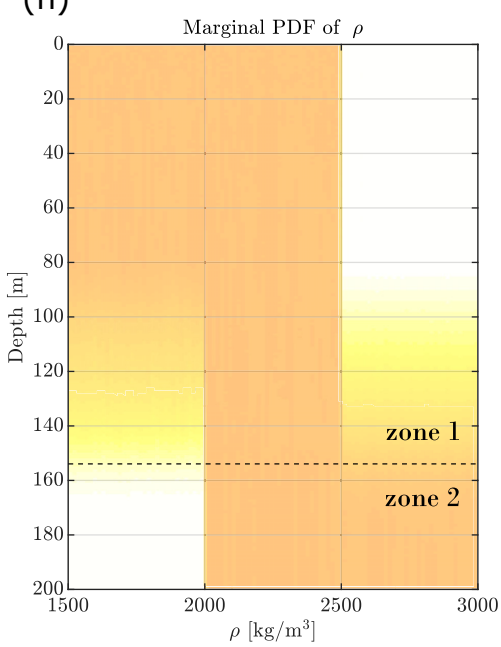

Figure 3. The apparent effective prior in the test with required positive velocity gradient (avoiding low-velocity zones by additional conditions). Figure layout and colourscale are the same as in Fig. 2.

prior on $S$-wave velocity in Fig. 2(b) and mass density in Fig. 2(d) are uniform, while the effective prior on $P$-wave velocity (Fig. 2c) is weakly affected by the plausible Poisson's ratio (see model space thresholds in Table D1 of Appendix D). The reciprocal distribution $p(k) \propto k^{-1}$ prescribed for the number of layers is preserved in the effective prior (Fig. 2a). This test's results are particularly satisfactory: the effective prior of $v_{\mathrm{S}}$ is uniform within the full parameter range because of the implemented random walk (see Supplement S2); the reversible-jump algorithm behaves exactly as expected (see Supplement S3); $v_{\mathrm{S}}$ and $\rho$ are uniform and not affected by the condition on the Poisson's ratio as indicated by eq. (16). Further, the effective prior on the multizonal model space follow the pattern just described (see bottom panels in Fig. 2). Besides, the priors of VNi parameters are prescribed as zone-specific, resulting in denser effective prior PDF. Note that the effective prior is smooth in proximity of the $z$-interfaces (see Fig. 2f). It occurs because, in the parametrization, the layer boundaries are defined as equidistant between two adjacent $\mathrm{VNi}$, but the multizonal prior is prescribed for $\mathrm{VNi}$ themselves (see Fig. 1b, showing how the layer boundaries are not bound to the $z$-interfaces). We consider the smooth prior as a convenient advantage feature because it allows treating the zones as generic uncertain stratified geological units rather than as layers with fixed thickness.

Next, the apparent effective prior in the model space with required positive velocity gradient is shown in Fig. $3\left(h^{\text {thr }}=h^{\text {min }}\right.$ settings of the computational code). The effective prior is further complicated; nevertheless, the reciprocal distribution (Figs 3a and e) and the prior on the mass density (Figs $3 \mathrm{~d}$ and $\mathrm{h}$ ) are unaltered. Note that the apparent effective prior is complex due to additional interlayer conditions on the velocity gradient, which is not directly visible in the marginal distribution. Also note that the complex effective prior in Fig. 3(b) may indirectly reveal the resolution limit of the observed data at depth (i.e. an inferred posterior marginal PDF similar to the effective prior would indicate no resolution of the observed data). The last, the apparent effective prior on the multizonal model space is very complex when the gradient condition applied (bottom panels in Fig. 3). Hence, this particular combination of additional conditions and prior have to be avoided. 
Table 1. Parameters of the 1-D model used as the target model of synthetic tests.

\begin{tabular}{lccc}
\hline Top depth $[\mathrm{m}]$ & $\begin{array}{c}v_{\mathrm{S}} \\
{\left[\mathrm{m} \mathrm{s}^{-1}\right]}\end{array}$ & $\begin{array}{c}v_{\mathrm{P}} \\
{\left[\mathrm{m} \mathrm{s}^{-1}\right]}\end{array}$ & $\rho\left[\mathrm{kg} \mathrm{m}^{-3}\right]$ \\
\hline 0 & 200 & 360 & 1800 \\
20 & 450 & 810 & 1950 \\
70 & 1000 & 1800 & 2000 \\
160 & 2000 & 3600 & 2700 \\
\hline
\end{tabular}

\subsection{Processing of the ensemble of solution}

The MTI computational program produces an ensemble of models drawn from the posterior PDF. This ensemble is then used to construct 2-D marginal histograms of the model parameters $v_{\mathrm{S}}, v_{\mathrm{P}}$ and $\rho$ with respect to the profile depth (e.g. Figs 2 and 3). Additionally, we can compute for each model of the ensemble $v_{\mathrm{S} 30}$, QWL representation, and theoretical SH-wave transfer function (e.g. Boore 2003) and construct their marginal histograms. For example, we can inspect histograms of $v_{\mathrm{S} 30}$ as captured by the whole ensemble of solutions (containing plausible models of varying number of layers). Note that a marginal distribution do not show the full effect of the high-dimensional model space, it is just 2-D statistics.

Marginal histograms are useful to obtain information on the uncertainty of the parameters of interest. Further, following Bodin et al. (2012), we construct the marginal posterior maximum profile (MAX of PDF) by taking the most frequent value of a property ( $v_{\mathrm{S}}, v_{\mathrm{P}}$ or $\rho$ ) at each discrete depth across the ensemble of solutions. We also define the marginal posterior average profile (AM of PDF) accompanied by its uncertainty by taking the arithmetic mean and the standard deviation of a property $\left(1 / v_{\mathrm{S}}, 1 / v_{\mathrm{P}}\right.$ or $\left.\rho\right)$ at each discrete depth across the ensemble of solutions. Note that these two profiles are merely statistical properties of the marginal posterior PDF, and therefore they do not correspond to any particular 1-D model with an assigned data fit.

Although the result of the Bayesian inversion is the posterior PDF, a single representative 1-D model is usually required. For this purpose, we can define the maximum likelihood model (ML model) as the model with the lowest misfit (expressed in the joint standardized units) between observed and modelled data. The ML model is representative in terms of data fit, but it may be misleading for inversions with a non-unique solution. Finally, we minimize the misfit, measured in the L1-norm in discrete ln-depths, between the MAX of PDF of a velocity (both $v_{\mathrm{S}}$ and $v_{\mathrm{P}}$ with weights 1.0 and 0.5 , respectively) and models from the ensemble to approximate the maximum a posteriori model (MAP model). Although merely an estimate of the MAP model, it is representative in terms of the posterior PDF of $v_{\mathrm{S}}$ (primarily) and $v_{\mathrm{P}}$ (secondarily).

\section{VALIDATION OF THE INVERSION PROCEDURE}

In this section, we demonstrate our approach's validity by inverting synthetic data referred to a 'target model'. The primary inference was set-up as a blind test, where the target velocity model in Table 1 was unknown to the co-author running the inversion (i.e. 'interpreter') to minimize any possible bias. The interpreter was only provided with the model space limits and an estimated bedrock depth in the interval 154 $\pm 10 \mathrm{~m}$.

\subsection{Data and inversion settings}

The synthetic data (used as the observed data in the inversion) were computed by Geopsy software, solving the forward problem in the 1-D velocity model from Table 1 . The vector of observed data consists of fundamental and first higher modes of Rayleigh waves (frequency range 0.8-20 Hz and 1.6-25 Hz, respectively), fundamental mode of Love waves (frequency range 0.8-20 Hz), and ellipticity of the fundamental mode of Rayleigh waves (frequency range 0.3-20 Hz). Apart from the observed data, we assumed the vector of the standard deviation of theoretical data errors as large as 10 per cent of the current slowness value and $0.04-0.38$ of the common logarithm of ellipticity. The synthetic observed data are noise-free; the assumed standard deviations of data errors are only to simulate real uncertainties. These data and uncertainties are shown in Figs 4(a)-(d) by black curves and error-bars, respectively.

We run two main independent tests, first assuming the single-zone transdimensional model space (see model space thresholds in Table D1 of Appendix D) and then the multizonal one consisting of two zones with an interface depth of $154 \mathrm{~m}$ (see model space thresholds in Table D2 of Appendix D). We apply no constraints on the velocity gradient for both tests (i.e. $h^{\text {thr }}=h^{\text {max }}$ ). Hence, low-velocity zones are allowed everywhere in the $200 \mathrm{~m}$ long profile, and the effective priors are just as shown in Fig. 2. Each ensemble of models drawn from the posterior PDF resulted from 460 Markov chains of the Parallel Tempering (437 exploration and 23 sampling chains). For each chain, the length of burn-in and production phases were set to 5000 and $\sim 25000$ steps, respectively, corresponding to $\sim 13800000$ models tested and $\sim 575000$ random samples drawn from the posterior PDF. 
(a)

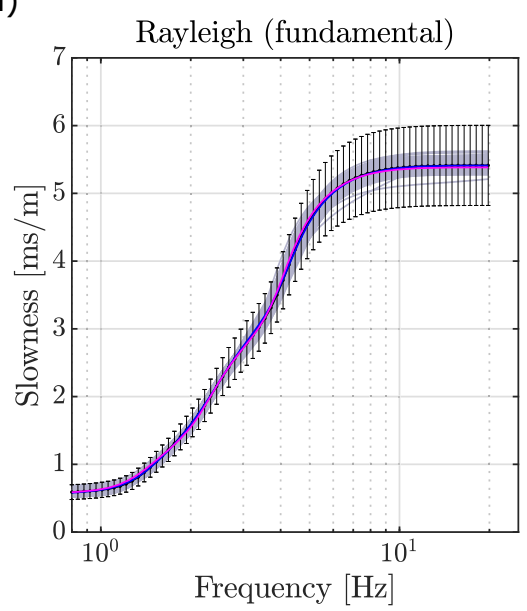

(d)

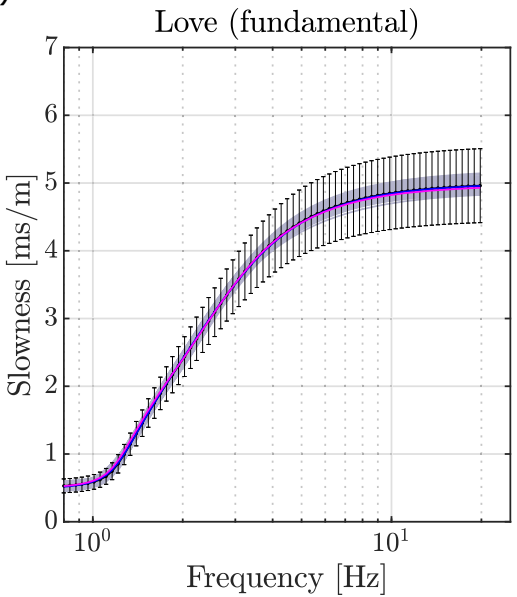

(b)

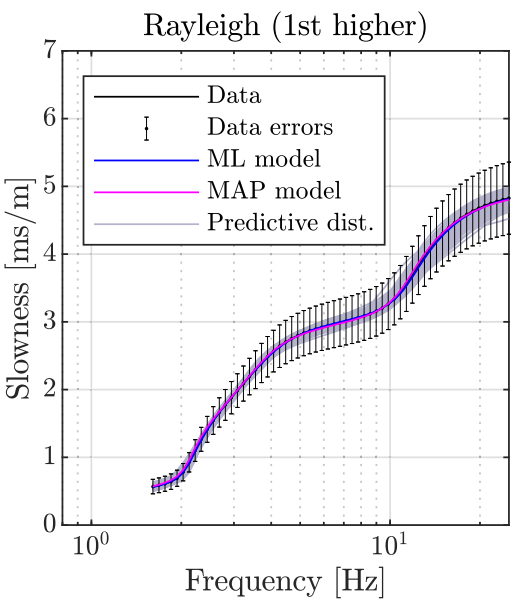

(e)

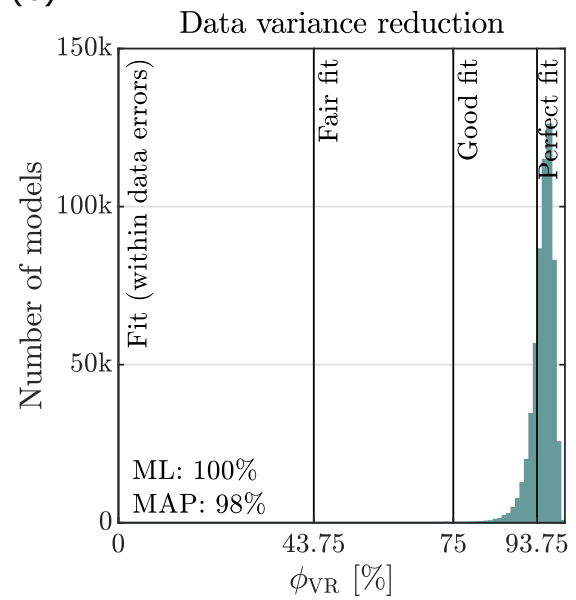

(c)

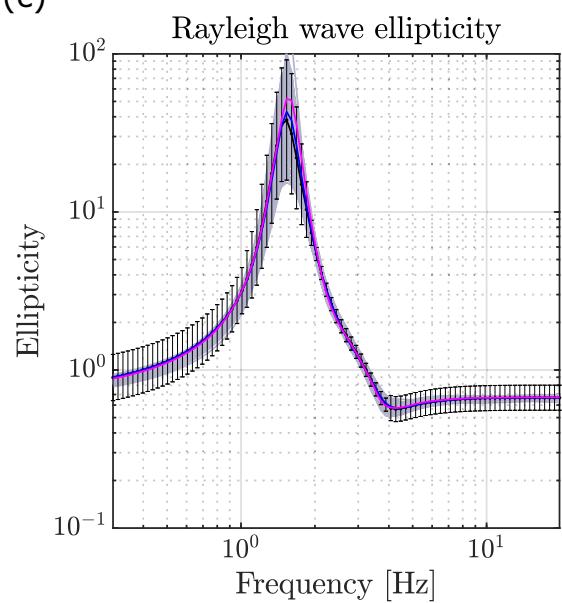

(f)

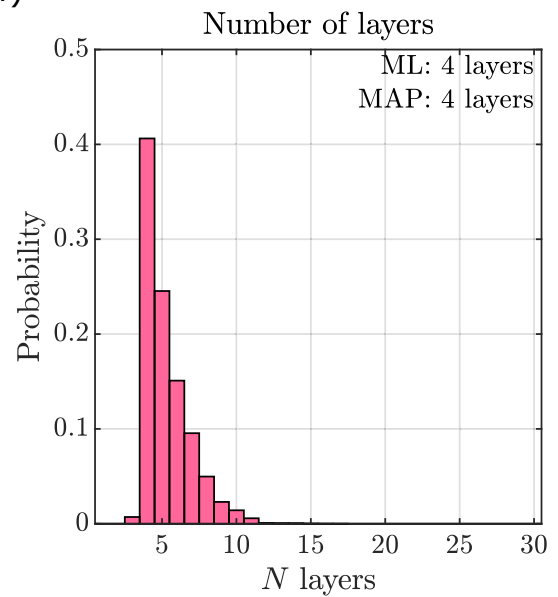

Figure 4. Fit of the observed (synthetic) and modelled data from the inversion with the single-zone transdimensional model space. Data fit in panels (a), (b), (c) and (d) is almost perfect, and hence, the observed data (black curves with error-bars) are overlayed by the posterior predictive distribution (grey), ML model (blue) and MAP model (magenta). Panels (e) and (f) show histograms for data variance reduction and number of layers, respectively.

\subsection{Single-zone transdimensional inference}

The inference in the single-zone transdimensional model space (i.e. a special case of multizonal inference having $Z=1$; see eq. (1)) is suitable when no information on the subsurface is available a priori. The inference is expected to be similar to a standard transdimensional inversion; however, it is enhanced in our approach using the ln-depth domain of the model space, the first-order mirroring of the proposal distribution at model space edges, and the self-adapting complexity of inferred models (see Section 2 for details). We have inferred posterior PDF in such a model space, and we show results in Figs 4, 5 and 6.

Since we do not add any artificial noise to the observed (synthetic) data, one would expect to find a model with zero data misfit (i.e. data variance reduction 100.0 per cent). However, strictly speaking, the probability of hitting just the target during a random exploration of a continuous model space is mathematically zero. Hence, modelled data are distributed close (yet not equal) to the target values (Figs 4a-d), and the data variance reduction is slightly lower than 100.0 per cent (Fig. 4e). For instance, the data variance reductions for ML and MAP models are 99.6 and 97.8 per cent, respectively. In our formulation, the number of layers is self-adapting and subject of the inversion itself. The histogram of all layered models in the ensemble of solutions shows a maximum probability of having four layers (Fig. 4f), which correspond to the correct value. It validates the self-adapting mechanism's functionality, where models are as simple as possible, but not simpler than as required by the observed data.

Results in terms of the posterior marginal PDF on the model space are shown in Fig. 5. For $v_{\mathrm{S}}$ and $v_{\mathrm{P}}$ the posterior PDF (Figs $5 \mathrm{a}$ and $\mathrm{b}$, respectively) is narrow in the uppermost two layers and broad below a depth of approximately $70 \mathrm{~m}$. It indicates that the model parameters are well resolved in the shallowest part of the profile, but there is a significant uncertainty in the deepest part. This is also evident when comparing the ML and MAP models with the target model, as they coincide only in the upper part (see Figs 5a and b). Note that these models appear on the left edge of the probable zone between 20 and $70 \mathrm{~m}$, as the posterior marginal PDF is asymmetrical (see MAX of PDF in Fig. 5e). The posterior marginal PDF of $\rho$ (Fig. 5d) is completely flat, which means that this parameter cannot be resolved in this test. Inferred values of $\rho$ for the ML and MAP models are therefore random and have no predictive value. In Fig. 5(c), we show a histogram for the layer interfaces 
(a)

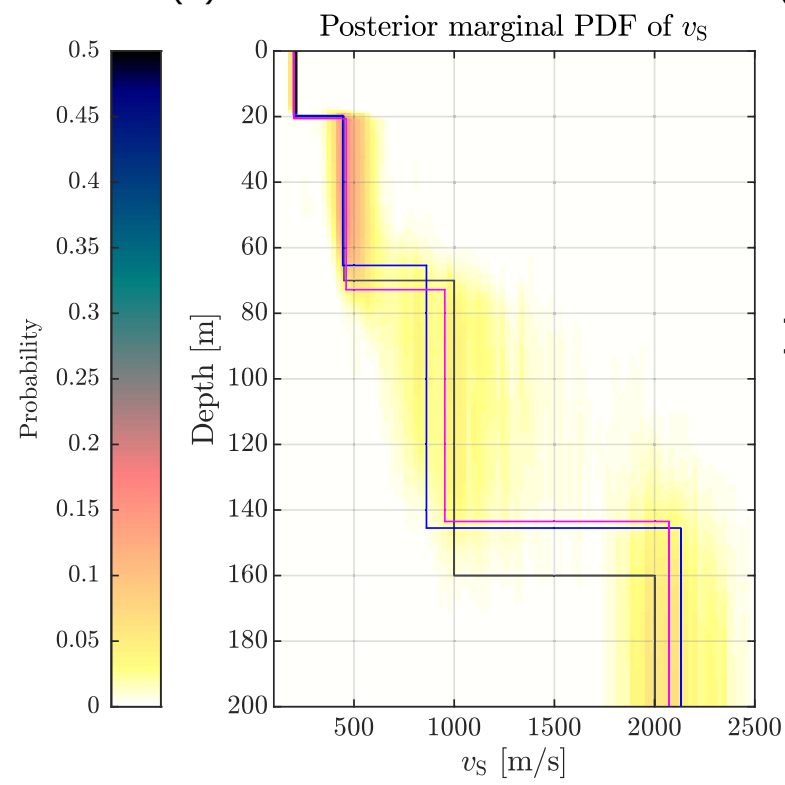

(b)

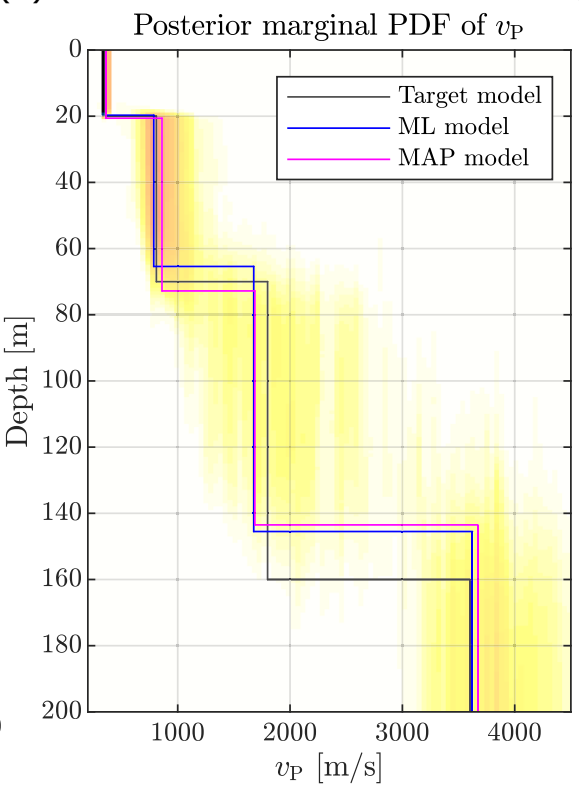

(c)

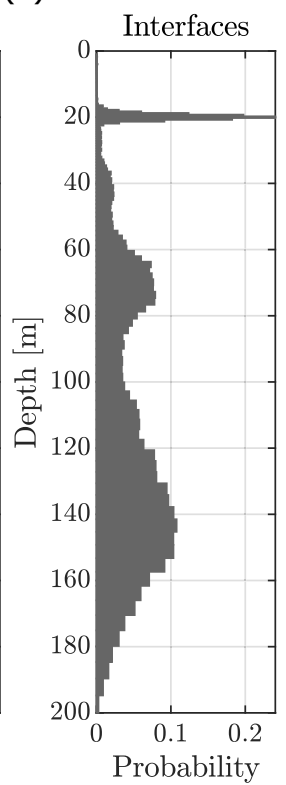

(d)

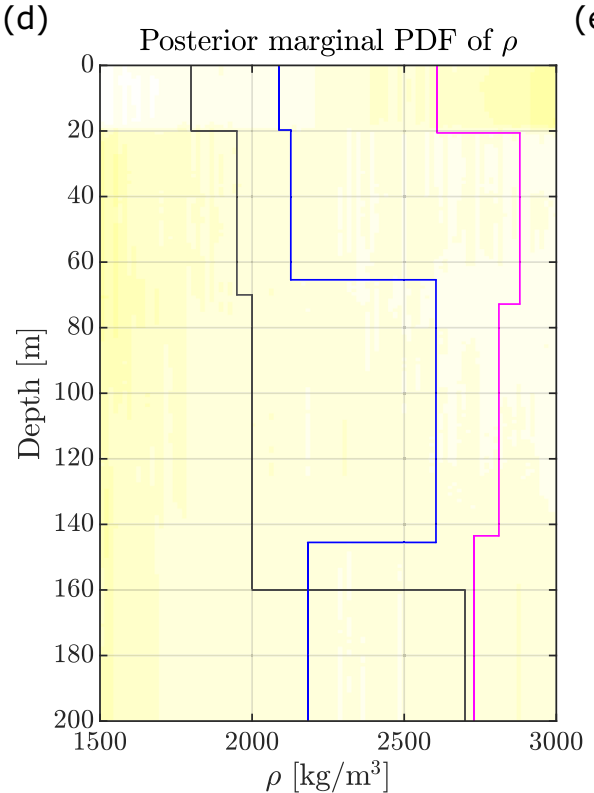

(e)

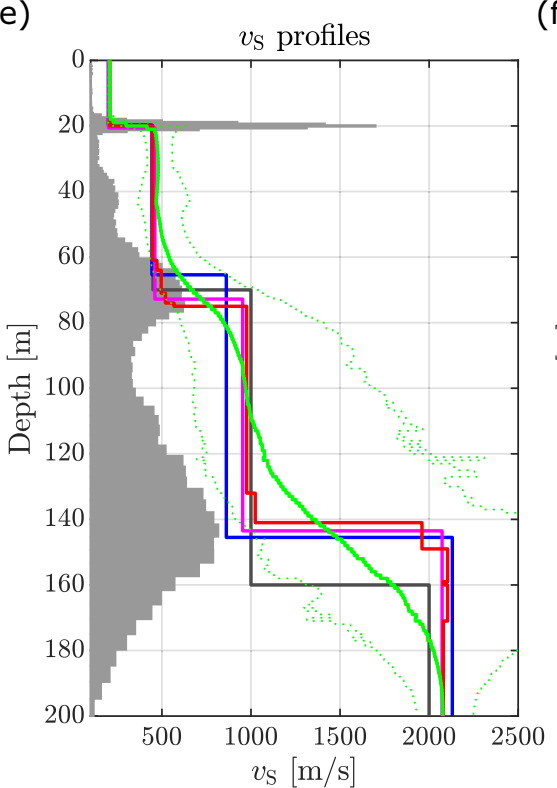

(f)

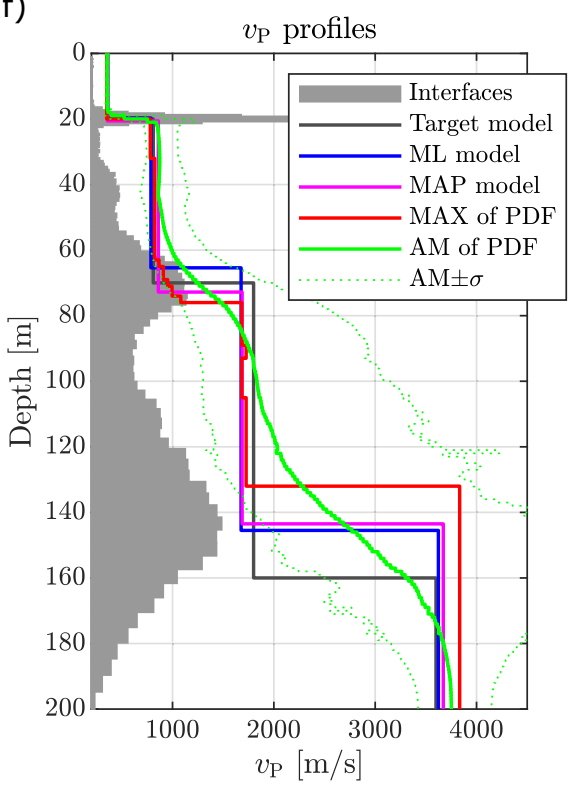

Figure 5. Inversion of synthetic data with the single-zone transdimensional model space. Panels (a), (b) and (d) show the posterior marginal PDF (colourscale) overlayed by the ML, MAP and target models (see legend). Panel (c) shows a histogram for the interface depth. Panels (e) and (f) show the inferred $S$ - and $P$-wave velocity profiles, respectively.

in depth. This histogram is constructed in the ln-depth domain (as it is the actual domain of the model space) and then transferred into the histogram with unequal bin sizes in the depth domain (as preferred for visualization purposes). The histogram in Fig. 5(c) indicates three primary interfaces at a depth of approximately 20,70 and $145 \mathrm{~m}$, and that there is a considerable uncertainty for the deepest interface. To better assess the inversion results' quality, we show a direct comparison of the velocity profiles in Figs 5(e) and (f). To recapitulate on profiles meaning: the target model was used for computing the observed (synthetic) data, the ML model provides the lowest data misfit, the MAP model is the most probable, MAX of PDF is a profile of the most frequent values in the ensemble of solutions, and AM of PDF is a profile of average values of the ensemble of solutions (preserving traveltime of a vertically propagating wave). Although all the profiles are consistent with the target model, the depth of the deepest interface (characterized by considerable uncertainty) is systematically smaller than expected (see Figs 5e and f). This feature may be explained by using parametrization in the ln-depth domain, which privileges the resolution in the shallowest part of the profile. Also note that the AM of PDF is very smooth at a depth of the deepest interface due to uncertainty (significant variance among models of the ensemble). 
(a)

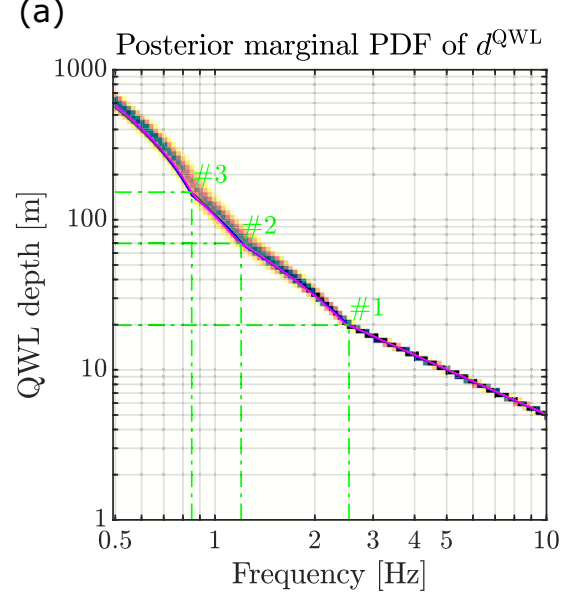

(d)

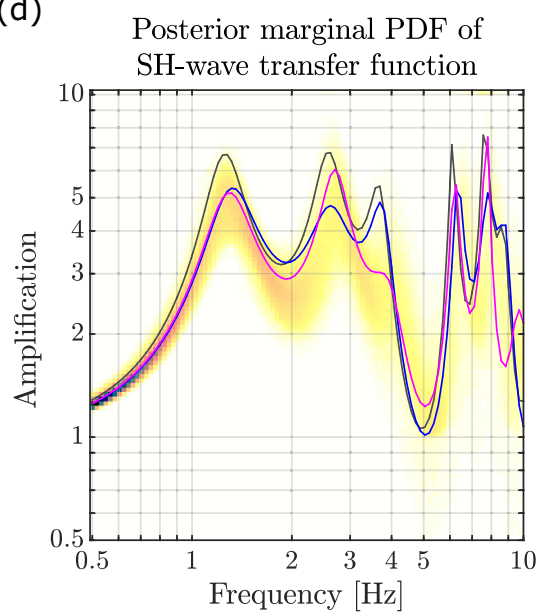

(b)

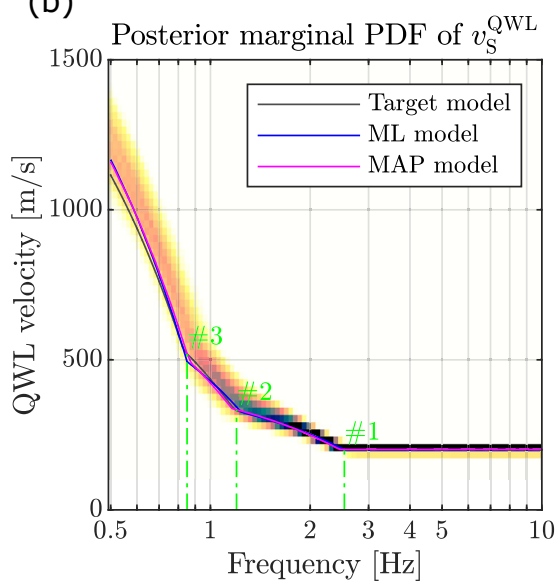

(e)

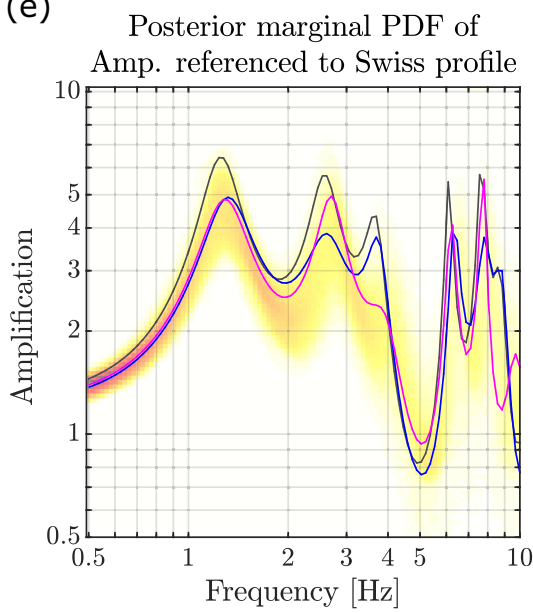

(c)
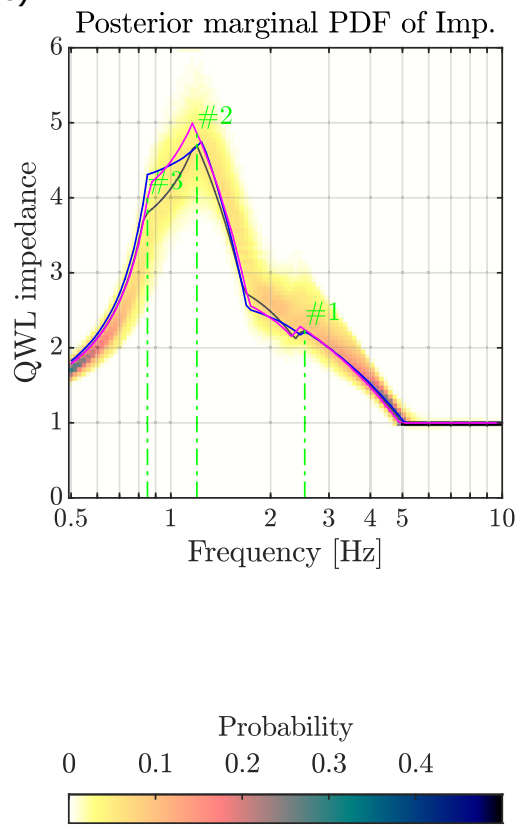

(f)

$$
\begin{array}{ll}
v_{\mathrm{S} S 0}^{\text {Target }}=245[\mathrm{~m} / \mathrm{s}] & f_{30}^{\text {Target }}=2.05[\mathrm{~Hz}] \\
v_{\mathrm{S} 30}^{\mathrm{ML}}=247[\mathrm{~m} / \mathrm{s}] & f_{30}^{\mathrm{ML}}=2.06[\mathrm{~Hz}] \\
v_{\mathrm{S} S 0}^{\mathrm{MAP}}=245[\mathrm{~m} / \mathrm{s}] & f_{30}^{\mathrm{MAP}}=2.04[\mathrm{~Hz}] \\
v_{\mathrm{S} 30}^{\mathrm{MAX}}=256[\mathrm{~m} / \mathrm{s}] & f_{30}^{\mathrm{MAX}}=2.13[\mathrm{~Hz}] \\
v_{\mathrm{S} 30}^{\mathrm{AM}}=251[\mathrm{~m} / \mathrm{s}] & f_{30}^{\mathrm{AM}}=2.09[\mathrm{~Hz}] \\
241<v_{\mathrm{S} 30}^{\mathrm{AM}}<261 & 2<f_{30}^{\mathrm{AM}}<2.2
\end{array}
$$

Figure 6. Statistics from the ensemble of solutions (single-zone transdimensional inversion of synthetic data). Panels (a), (b) and (c) shown PDFs of QWL depth, QWL velocity and QWL impedance. Green lines indicate properties associated with QWL depths of 20, 70 and $160 \mathrm{~m}$ (interfaces \#1, \#2 and \#3, respectively). Panels (d) and (e) show PDFs of SH-wave transfer function and amplification referenced to the Swiss profile (Poggi et al. 2011). Panel (f) shows the values of $\mathrm{v}_{\mathrm{S} 30}$ and $\mathrm{f}_{30}$ (frequency corresponding to $30 \mathrm{~m}$ depth).

Further, the ensemble of solutions was used for computing statistics of $v_{\mathrm{S} 30}$, QWL representation, theoretical SH-wave transfer function (e.g. Boore 2003), and theoretical amplification referenced to the Swiss profile (Poggi et al. 2011). The latter may predict the site amplification for the empirical spectral modelling (Edwards et al. 2013). This is of great importance, as it is essential for constructing seismic hazard maps (Wiemer et al. 2016). The posterior marginal PDFs of the QWL depth $\left(d^{\mathrm{QWL}}\right), S$-wave velocity $\left(v_{\mathrm{S}}^{\mathrm{QWL}}\right)$ and QWL impedance (Poggi et al. 2012) are very sharp (see upper panels in Fig. 6), implying that the associated QWL representation has relatively small uncertainty. Indeed, the QWL representations of ML, MAP and target models overlap. The posterior marginal PDFs of the SH-wave transfer function and amplification referenced to the Swiss profile (Figs 6d and e, respectively) involve some discrepancies in amplitudes with respect to the target model. It might be assigned to the uncertainty of the inferred bedrock depth and velocity. In Fig. 6(f), we compare the values of $v_{\mathrm{S} 30}$ of the ML, MAP and target models $\left(v_{\mathrm{S} 30}^{\mathrm{ML}}, v_{\mathrm{S} 30}^{\mathrm{MAP}}\right.$ and $v_{\mathrm{S} 30}^{\text {Target }}$, respectively) and statistics from the ensemble of solutions. In particular, we compute the most frequent value $\left(v_{\mathrm{S} 30}^{\mathrm{MAX}}\right)$ and arithmetic mean $\left(v_{\mathrm{S} 30}^{\mathrm{AM}}\right)$ including standard deviation. All of these are accompanied by respective $f_{30}$ (i.e. frequency corresponding to $30 \mathrm{~m}$ depth). In this test, the ML, MAP and target models lead to similar $v_{\mathrm{S} 30}$ values being also in accord with statistics from the ensemble of solutions (see Fig. 6f).

\subsection{Multizonal transdimensional inference}

The multizonal formulation allows us to include additional prior assumptions and exclude unlikely models from the model space. In this inversion test assuming the multizonal model space, we take advantage of the provided (yet uncertain) estimate of depth to the bedrock and divide the model space into two zones ( $z$-interface set to $154 \mathrm{~m}$ while the actual bedrock depth is $160 \mathrm{~m}$ ). The zone above this $z$-interface is assumed to have elastic properties of soil-to-(soft)rock and the zone below properties of rock-to-(hard)rock (see Table D2 in Appendix D).

Results of the multizonal inference (Fig. 7) are consistent with those of the single-zone one. The data fit is almost identical to the single-zone inference case in Fig. 4, therefore not shown here to keep this paper compact. The data variance reduction of the ML and MAP model is 99.6 and 98.6 per cent, respectively. The posterior marginal PDFs of model parameters are consistent with the single-zone inference 
(a)

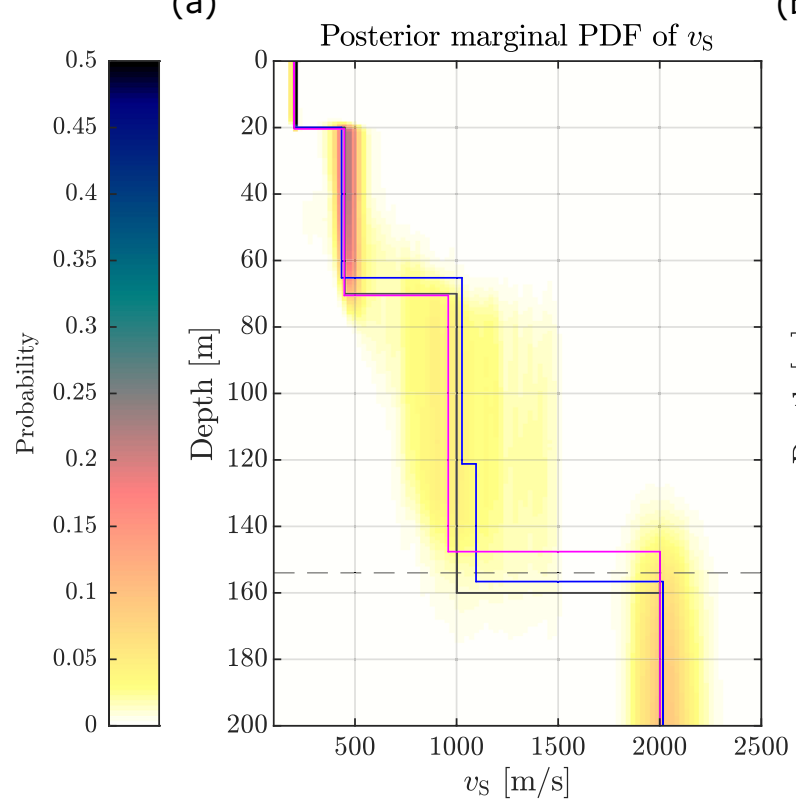

(b)

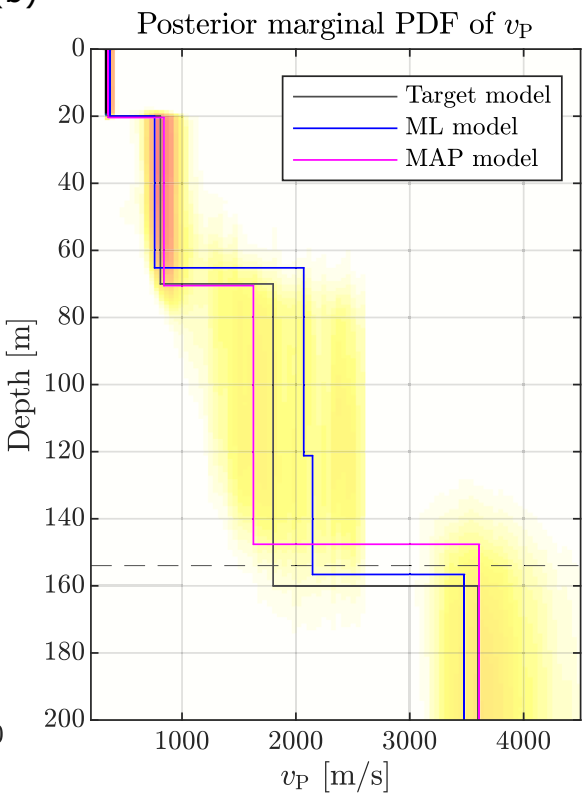

(c)

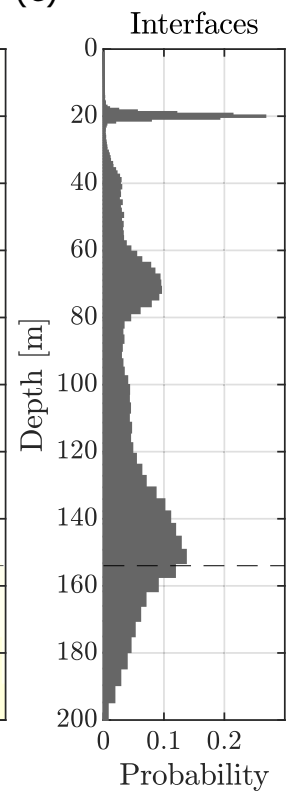

(d)

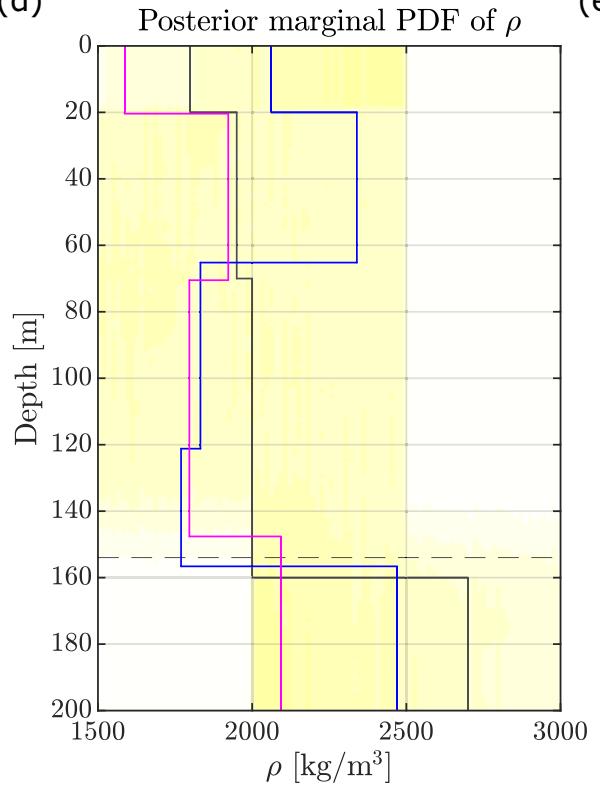

(e)

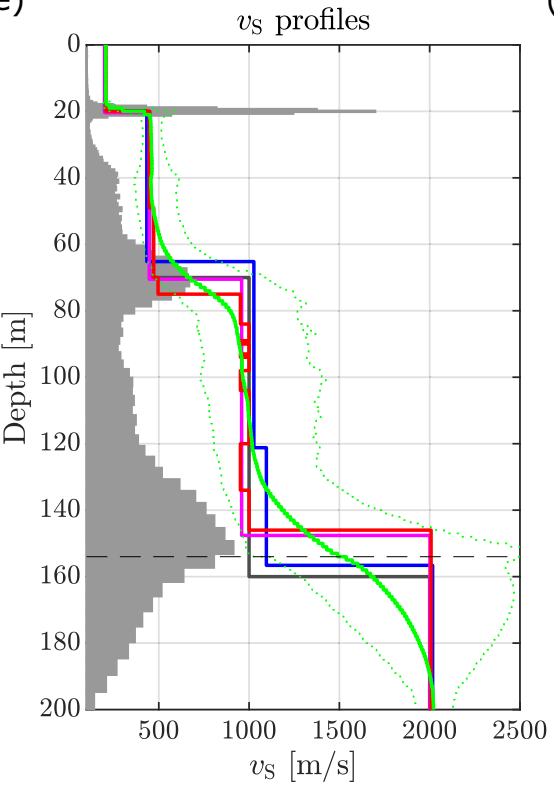

(f)

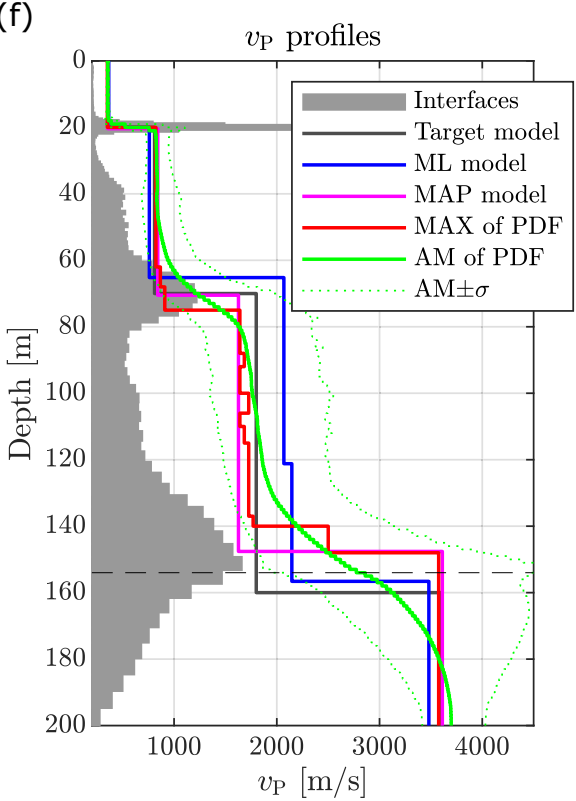

Figure 7. Inversion of synthetic data with the multizonal transdimensional model space. The $z$-interface (black dashed line) is placed at the depth 154 [m]. The figure layout is the same as in Fig. 5 .

(compare Figs 7 and 5), although some improvements can be noted. In particular, the posterior marginal PDF of $v_{\mathrm{S}}$ and $v_{\mathrm{P}}$ are sharper than in the single-zone inference case. The posterior PDF of $v_{\mathrm{S}}$ is now better resolved in the bedrock and its inferred depth is closer to the correct value of $160 \mathrm{~m}$. This is also confirmed by inspecting the ML, MAP and target models. The better resolution of the $v_{\mathrm{S}}$ and depth of the bedrock can also be noted in comparison of resultant velocity profiles (compare Figs 7e and 5e). Further, even if unlikely $\rho$ values are excluded from the model space in the multizonal inference, its posterior PDF remains flat (see Fig. 7d). The posterior marginal PDFs of the QWL representation remain sharp, and there are some improvements on low frequencies (compare upper panels in Figs 8 and 6). An improvement is notable for the SH-wave transfer function and amplification referenced to the Swiss profile (see Figs 8d and e) with respect to the single-zone inference. It is because spectral amplifications reflect properties along the whole profile (to $200 \mathrm{~m}$ depth) being better resolved in the multizonal case. The values of $v_{\mathrm{S} 30}$ (average velocity in the uppermost $30 \mathrm{~m}$ ) are in a similar range as in the single-zone inference (compare Figs $8 \mathrm{f}$ and $6 \mathrm{f}$ ). In particular, ML and MAP models have $v_{\mathrm{S} 30}$ equal to $244 \mathrm{~m} \mathrm{~s}^{-1}$ (Fig. 8f), which is in accord with the target model having $v_{\mathrm{S} 30}$ equal to $245 \mathrm{~m} \mathrm{~s}^{-1}$.

Additional prior assumptions of the multizonal inference may help to constrain the inverse problem better and improve results. However, incorrect assumptions may lead to the opposite effect and bias the solution. To further investigate this aspect, we show two additional multizonal inferences with incorrect prior assumptions in Appendix C. For the first test, we set the depth of the $z$-interface to an incorrect 
(a)

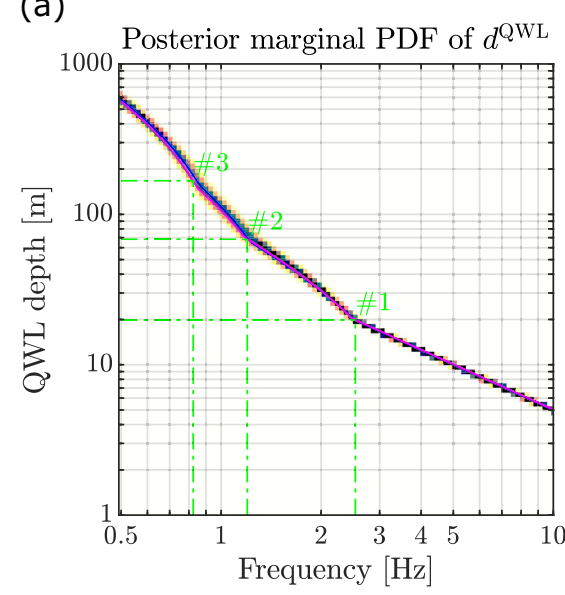

(d)

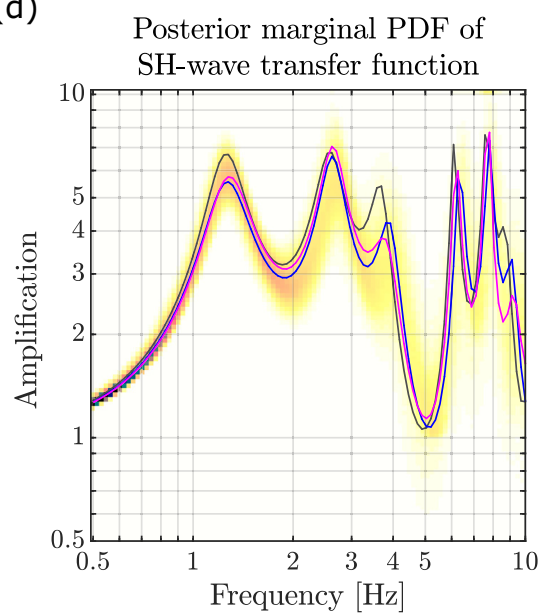

(b)

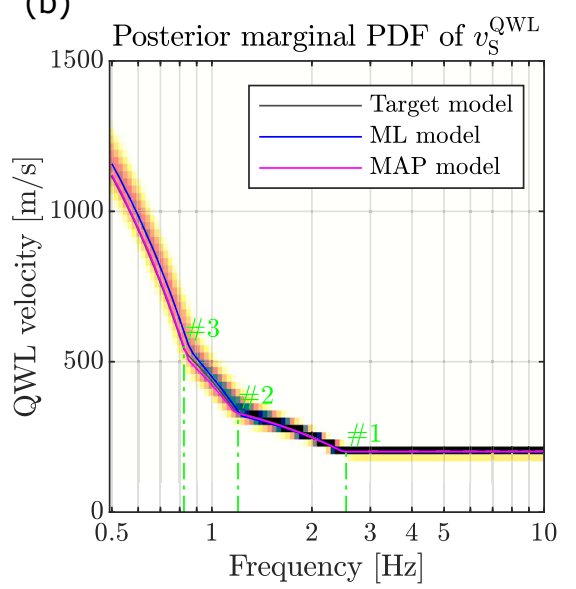

(e)

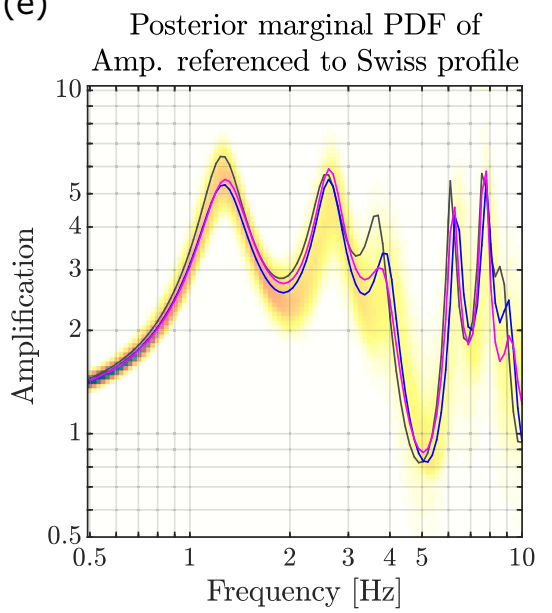

(c)

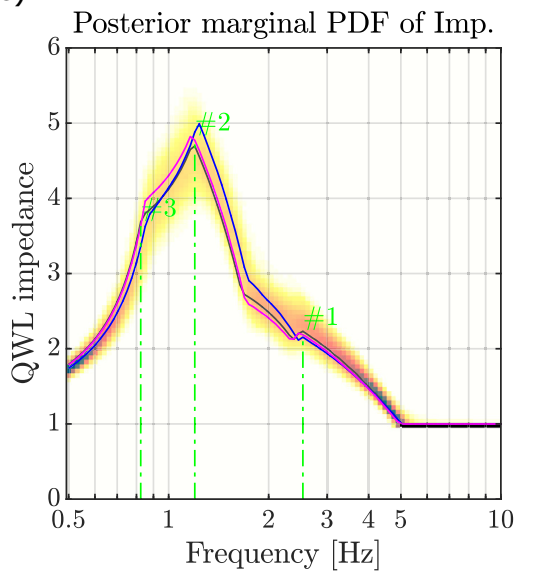

(f)

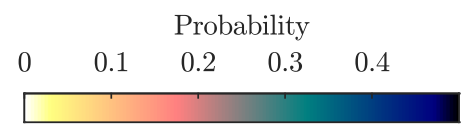

$$
\begin{array}{ll}
v_{\mathrm{S} 30}^{\text {Target }}=245[\mathrm{~m} / \mathrm{s}] & f_{30}^{\text {Target }}=2.05[\mathrm{~Hz}] \\
v_{\mathrm{S3}}^{\mathrm{MI}}=244[\mathrm{~m} / \mathrm{s}] & f_{30}^{\mathrm{ML}}=2.04[\mathrm{~Hz}] \\
v_{\mathrm{S3}}^{\mathrm{MAP}}=244[\mathrm{~m} / \mathrm{s}] & f_{30}^{\mathrm{MAP}}=2.04[\mathrm{~Hz}] \\
v_{\mathrm{S3}}^{\mathrm{MAX}}=256[\mathrm{~m} / \mathrm{s}] & f_{30}^{\mathrm{MAX}}=2.13[\mathrm{~Hz}] \\
v_{\mathrm{S} 30}^{\mathrm{MN}}=251[\mathrm{~m} / \mathrm{s}] & f_{30}^{\mathrm{MM}}=2.09[\mathrm{~Hz}] \\
241<v_{\mathrm{S} 30}^{\mathrm{AM}}<261 & 2<f_{30}^{\mathrm{AM}}<2.2
\end{array}
$$

Table 2. Parameters of the 1-D model with the velocity gradient.

\begin{tabular}{lccc}
\hline Top depth $[\mathrm{m}]$ & $v_{\mathrm{S}}\left[\mathrm{m} \mathrm{s}^{-1}\right]$ & $v_{\mathrm{P}}\left[\mathrm{m} \mathrm{s}^{-1}\right]$ & $\rho\left[\mathrm{kg} \mathrm{m}^{-3}\right]$ \\
\hline 0 & 200 & 360 & 1800 \\
20 & 450 & 810 & 1950 \\
$x+39$, where $x \in\{1, \ldots, 119\}$ & $4.55 x+450$ & $8.18 x+810$ & $0.41 x+1950$ \\
159 & 1000 & 1800 & 2000 \\
160 & 2000 & 3600 & 2700 \\
\hline
\end{tabular}

value of $100 \mathrm{~m}$ (see Fig. C1); and in the second test, we specify wrong velocity thresholds in the upper zone (see Fig. C2). Both inversions lead to a biased solution; nevertheless, a careful inspection of resultant PDFs may reveal such a bias (e.g. a sharp posterior marginal PDF at the edge of the zone-specific interval).

\subsection{Velocity gradients and low-velocity zones}

This section validates our inversion procedure on two more complex target models characterized by a continuous positive velocity gradient (Table 2) and a low-velocity zone (Table 3). The observed data vector consists of fundamental and first higher modes of Rayleigh waves, fundamental mode of Love waves, and Rayleigh wave ellipticity. We infer solutions assuming the single-zone transdimensional model space (see model space thresholds in Table D1 of Appendix D) and apply no constraints on the velocity gradient (i.e. $h^{\text {thr }}=h^{\max }$ ). Hence, low-velocity zones are allowed to occur everywhere along the profile. Note that assumed frequency ranges, data errors, and the model space exploration settings are identical to those in the previous sections.

The inversion of observed (synthetic) data prepared in the model with a velocity gradient (Table 2) is shown in Fig. 9. The posterior marginal PDFs of $v_{\mathrm{S}}$ and $v_{\mathrm{P}}$ (Figs 9a and $\mathrm{b}$ ) are narrow in the uppermost (homogeneous) layers and rather broad at depth where the gradient is located. The ML and MAP models possess an interface at approximately $80 \mathrm{~m}$ depth that does not correspond to the target model. This artificial interface compensates for the gradual velocity increase in the depth range $40-160 \mathrm{~m}$, and it is characterized by a high uncertainty 
Table 3. Parameters of the 1-D model with the lowvelocity zone.

\begin{tabular}{lccc}
\hline Top depth $[\mathrm{m}]$ & $v_{\mathrm{S}}\left[\mathrm{m} \mathrm{s}^{-1}\right]$ & $v_{\mathrm{P}}\left[\mathrm{m} \mathrm{s}^{-1}\right]$ & $\rho\left[\mathrm{kg} \mathrm{m}^{-3}\right]$ \\
\hline 0 & 200 & 360 & 1800 \\
20 & 1000 & 1800 & 2000 \\
40 & 450 & 810 & 1950 \\
70 & 1000 & 1800 & 2000 \\
160 & 2000 & 3600 & 2700 \\
\hline
\end{tabular}

(a)

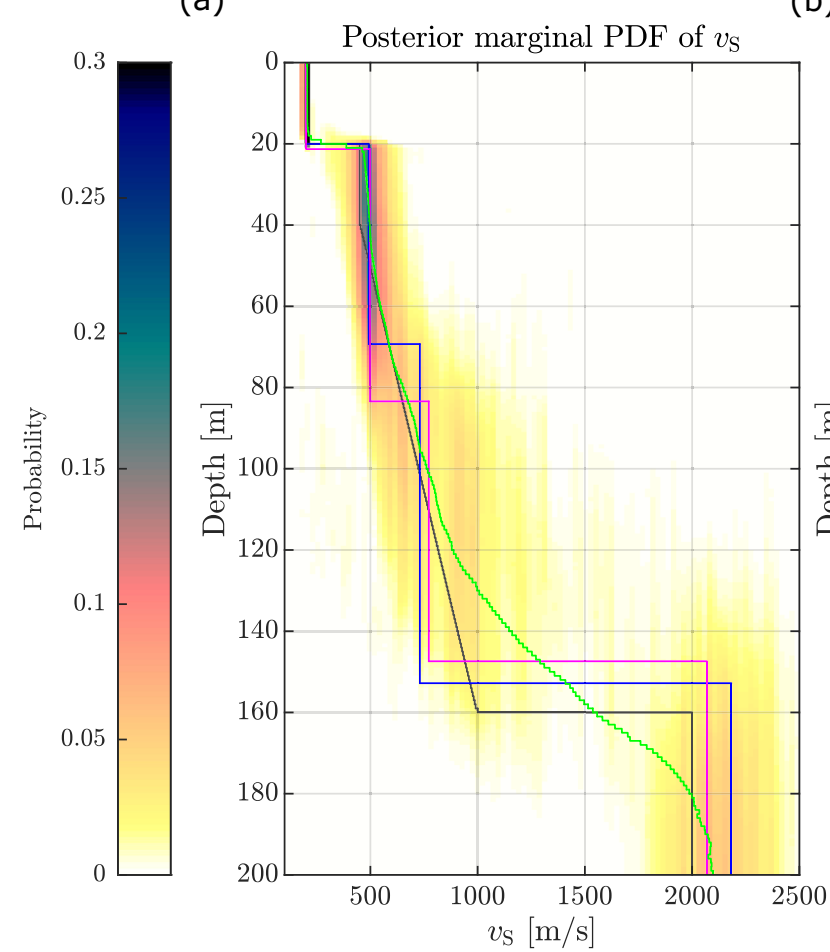

(b)

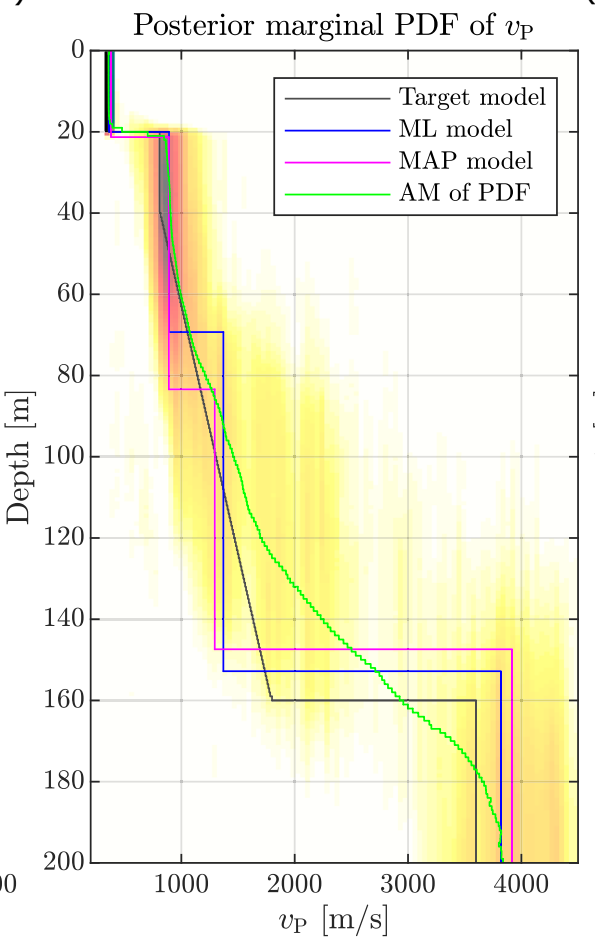

(c)

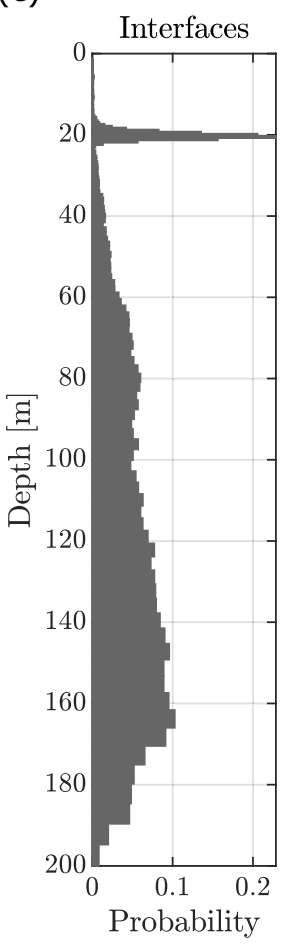

(d)
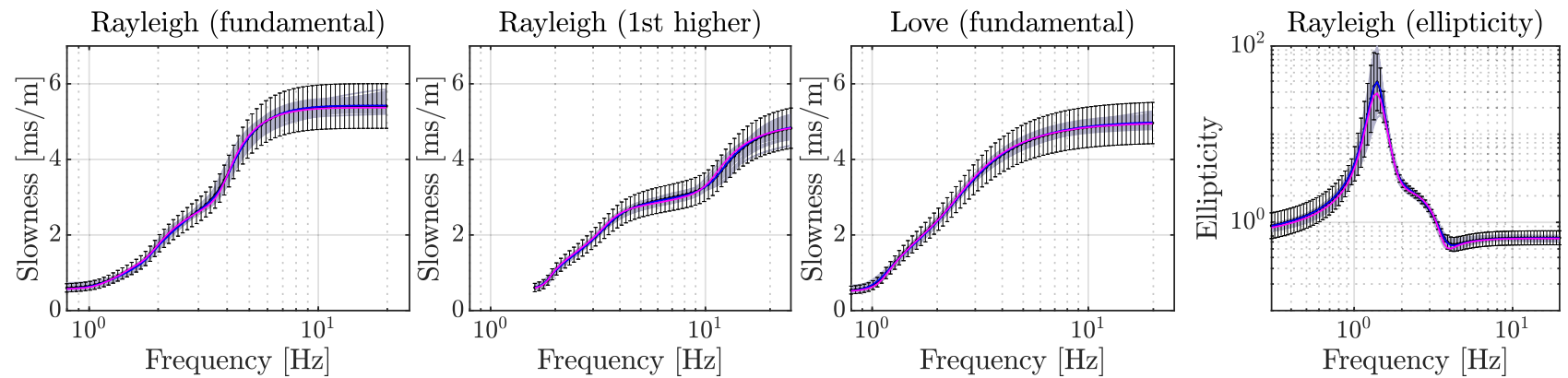

Figure 9. Inversion of synthetic data prepared in the model with a gradual velocity increase. Panels (a) and (b) show the posterior marginal PDF (colourscale) overlayed by the ML, MAP, and target models (see legend). Panel (c) shows a histogram for the interface depth. The bottom panels in (d) show the fit of the observed (synthetic) and modelled data.

(see Fig. 9c). Note that this results from the self-adapting number of layers; a large number of such fictitious interfaces may be expected if it significantly improves the data fit. Further, the AM of PDF profile provides a reliable estimate of the velocity gradient to approximately $120 \mathrm{~m}$ depth (see green line in Fig. 9a), and it is smoothed around the interface at a depth of $160 \mathrm{~m}$ (due to a significant variance among models). Thus, AM of PDF may represent sites with a smooth velocity gradient, still it may fail in imaging of subsurface consisting of layers with significant velocity contrasts. Finally, note that the inferred profiles do not possess low-velocity zones, even though they were allowed freely in this test.

In Fig. 10, we show the test results with a relatively thick low-velocity zone (see Table 3). Note that such a zone is not evident from visual inspection of dispersion curves (see Fig. 10d), and hence this inversion test is challenging. The posterior marginal PDFs of $v_{\mathrm{S}}$ and $v_{\mathrm{P}}$ (Figs 10a and b) as well as the ML and MAP models are ambiguous in this case. Nevertheless, the histogram in Fig. 10(c) clearly shows the 
(a)

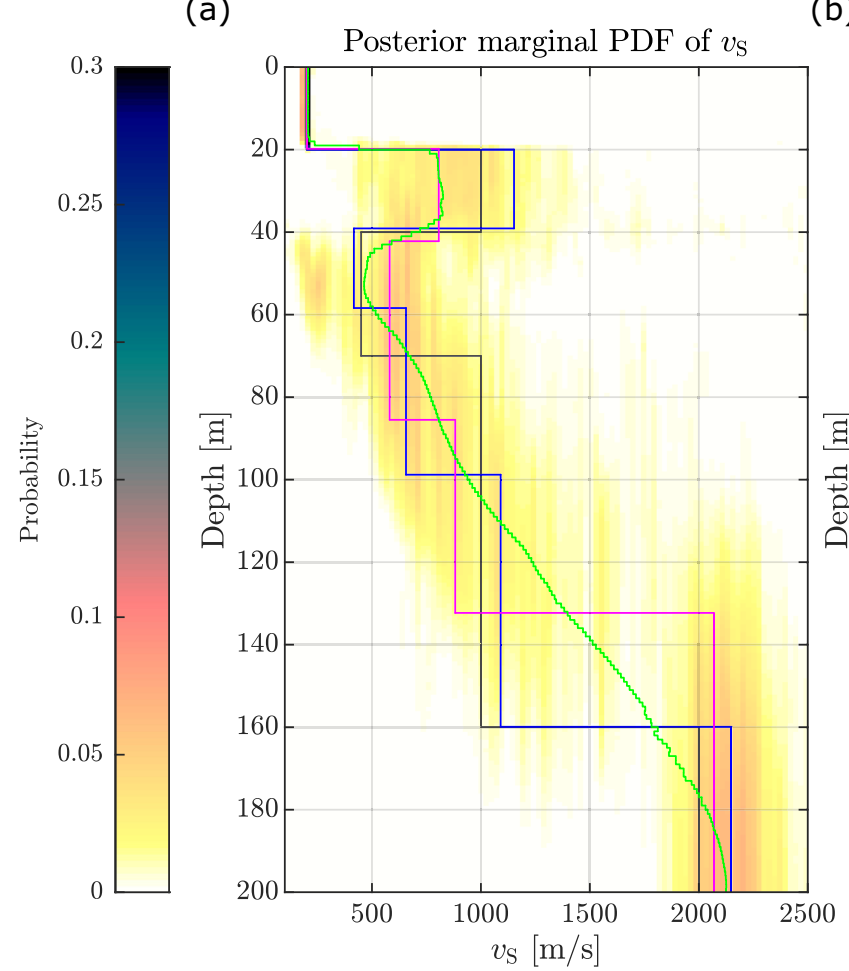

(b)

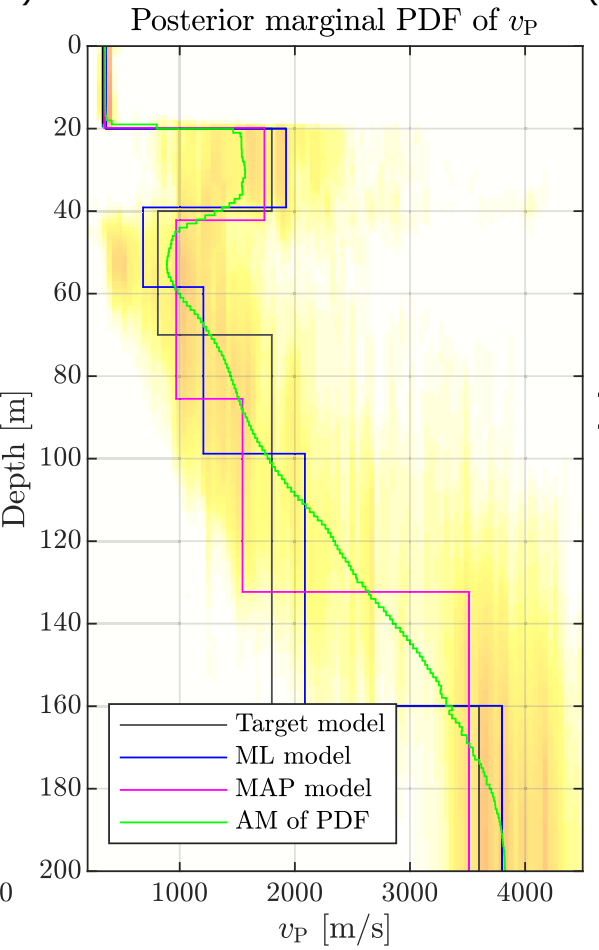

(c)

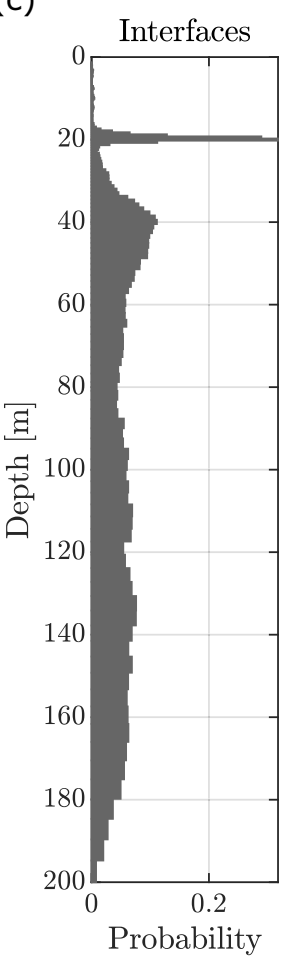

(d)
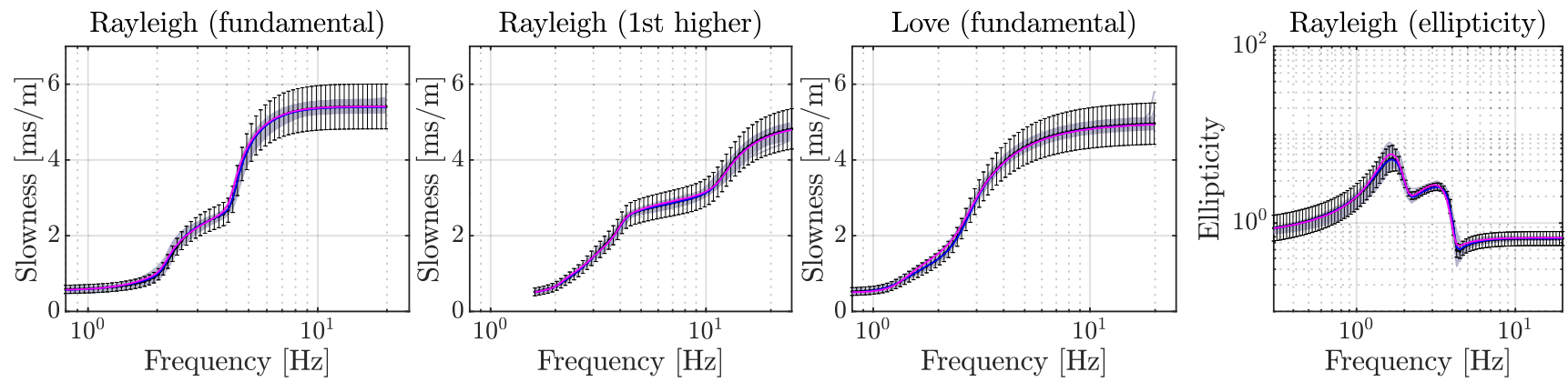

Figure 10. Inversion of synthetic data prepared in the model with a low-velocity zone. The figure layout is the same as in Fig. 9.

depth of the first interface and the interface where the velocity inversion begins (20 and $40 \mathrm{~m}$ depth, respectively), which means that the upper depth of the low-velocity zone can be resolved. The structure below is uncertain, as suggested by the broad PDF and smooth AM of PDF profile. The resolution is somewhat recovered for the bedrock at a depth of $160 \mathrm{~m}$. It suggests that interfaces delimiting velocity inversion act as shadow zones making the structure immediately below hardly resolvable. Still, it is clear that we cannot explain the observed data in Fig. 10(d) without the presence of a low-velocity zone below $40 \mathrm{~m}$ depth.

\section{REAL TEST CASE: APPLICATION TO A SITE IN CENTRAL SWITZERLAND}

In this section, we apply our inversion method to real surface wave data from the active seismic survey and single-station measurements of ambient vibrations. Measurements were performed at the site of SENGL seismic station belonging to the Swiss Strong Motion Network (SSMNet; Hobiger et al. 2016). We chose this specific site because of the large amount of available data: three modes of Rayleigh and two modes of Love wave dispersion curves from active survey and Rayleigh wave ellipticity estimated from single-station ambient seismic noise measurements.

The seismic station SENGL is located in central Switzerland (see Fig. 11) in an alpine valley at an altitude of $1033 \mathrm{~m}$. This station's site is characterized by conoid deposits (gravel) with a shallow detritus cover (soil organic material). The conoid deposits are laterally surrounded by a landslide, moraine and alluvial deposits (Swisstopo 2019). Soft sediments are estimated to have a thickness of approximately 10-15 $\mathrm{m}$, and the hard rock bedrock is expected at a depth of roughly $80 \mathrm{~m}$ (Swisstopo 2015). Our inversion focuses on the uppermost $50 \mathrm{~m}$ of this site. 


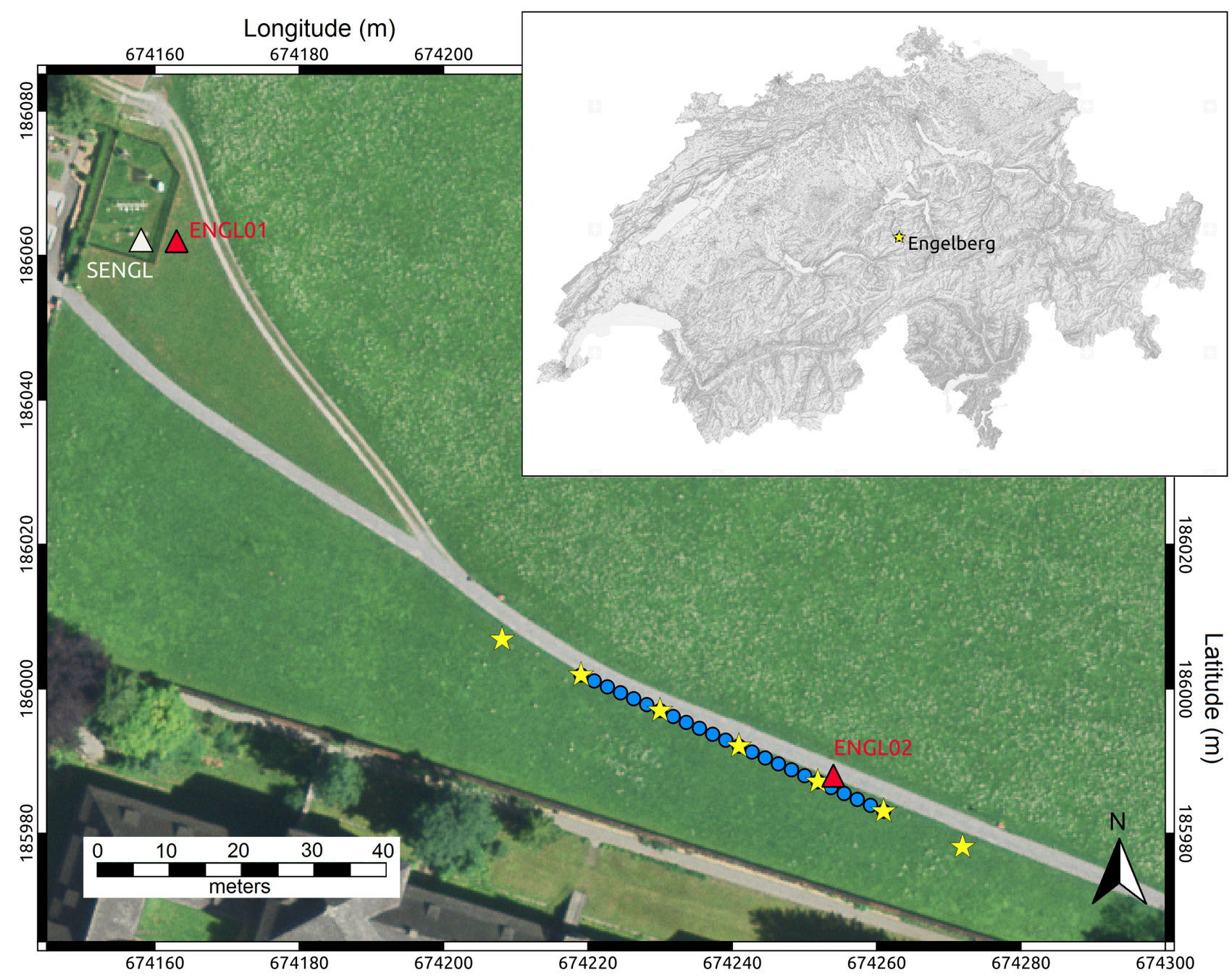

Figure 11. Measurement geometry at the site of the SENGL seismic station (Engelberg). The map shows the position of the SENGL seismic station (white triangle), active seismic line (blue circles - geophones, yellow stars - sources), and ambient noise recording sensors (red triangles). Longitude and Latitude are in Swiss CH1903/LV03 coordinate reference system EPSG:21781. The overview map shows the location of the Engelberg municipality within Switzerland.

\subsection{Surface wave data}

The active seismic survey (MASW; Park et al. 1999) and single-station measurements of ambient vibrations were carried out in the framework of the SSMNet renewal project (Hobiger et al. 2016). The active seismic measurement was realized about $100 \mathrm{~m}$ far away from the SENGL seismic station using one seismic line of three-component geophones (see Fig. 11). Rayleigh and Love wave dispersion curves were obtained from measurements by a processing in the $f-k$ domain. The single-station measurement of ambient vibrations was then performed using a three-component broadband seismometer near the active seismic line (ENGL02 sensor in Fig. 11). The ellipticity of Rayleigh waves was estimated from the measured data by the RayDec method (Hobiger et al. 2009). The complete description of the measurement and data analysis at the SENGL site is summarized in Panzera et al. (2020).

The surface wave data (input data of the inversion) consist of fundamental, first higher, and second higher modes of Rayleigh waves; fundamental and first higher modes of Love waves; and ellipticity of the Rayleigh waves (frequency ranges 8.8-40 Hz, 12-45 Hz, 26$43 \mathrm{~Hz}, 7.8-54 \mathrm{~Hz}, 24-55 \mathrm{~Hz}$ and $2.0-26 \mathrm{~Hz}$, respectively). The data are accompanied by uncertainties (vector of standard deviations of data errors) estimated from stacked $f-k$ panels (dispersion curves) or as an output from the RayDec method (ellipticity). The real data, including uncertainties, are shown in Fig. 12 by black curves and error-bars.

\subsection{Inversion of the real data}

For this application, we use the single-zone transdimensional model space, as no reliable information on the subsurface is available a priori (see model space thresholds in Table D3 of Appendix D), and we constrain the velocity gradient to be positive (i.e. $h^{\text {thr }}=h^{\text {min }}$ ). This means that the adapted effective prior would resemble the effective prior shown in Fig. 3 (upper panels). The ensemble of models was produced using 300 Markov chains of the Parallel Tempering (285 exploration and 15 sampling chains). For each chain, the length of the burn-in 
(a)

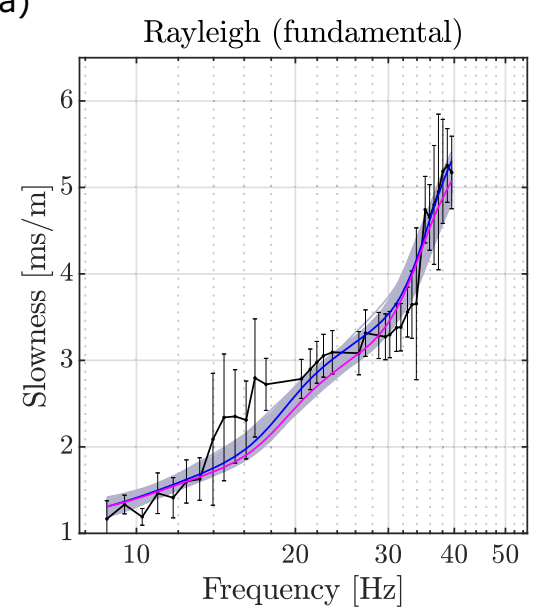

(d)

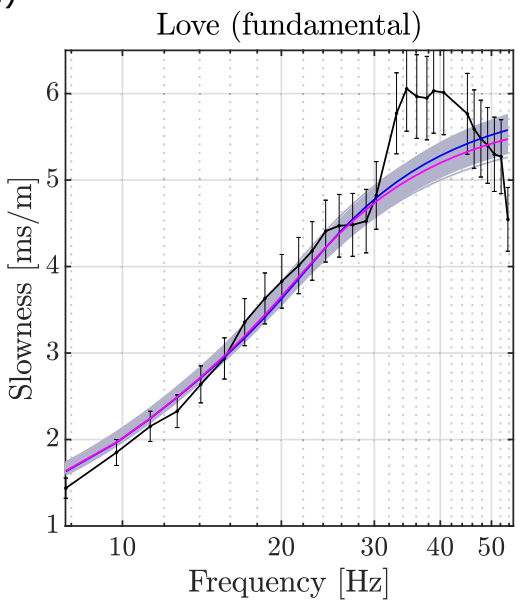

(b)

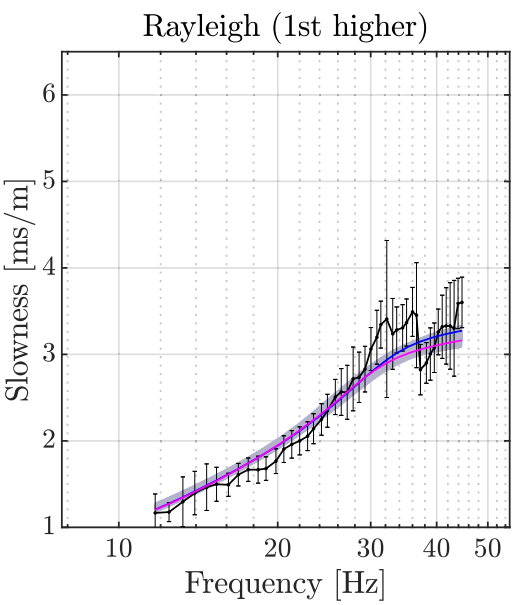

(e)

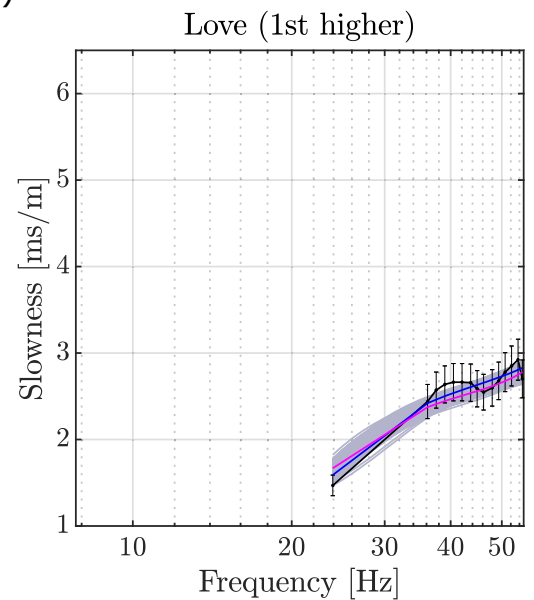

(c)

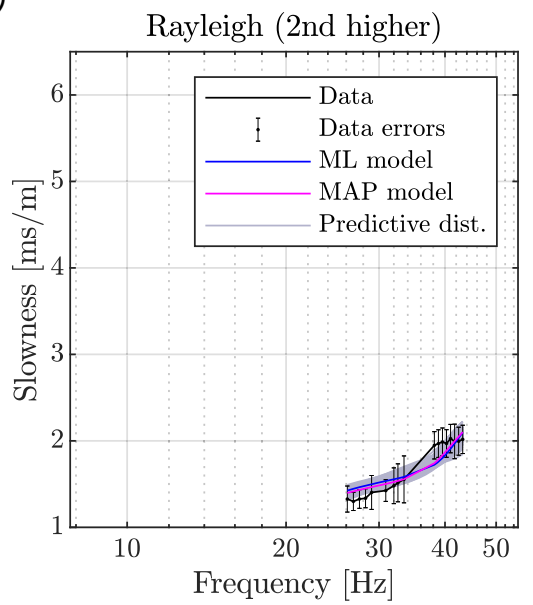

(f)

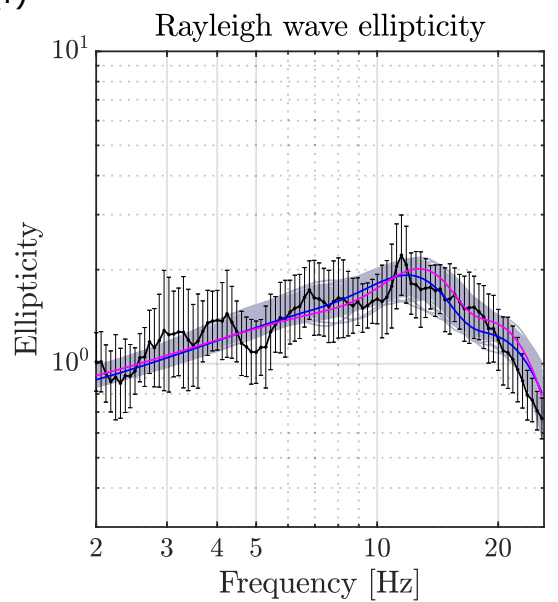

Figure 12. Fit of the real data measured at the SENGL site (black curves with error-bars) and data modelled in velocity models from the ensemble of solutions. Panels (a)-(e) show data retrieved by the MASW method (joint axes are used). Panel (f) shows data retrieved by the RayDec method (Hobiger et al. 2009) from single-station measurements of ambient vibrations.

and production phases was set to 5000 and $\sim 40000$ steps, respectively, corresponding to $\sim 13500000$ models tested and $\sim 600000$ random samples drawn from the posterior PDF.

The data fit, shown in Fig. 12, is satisfactory for a joint inversion of six different data types (five times slowness and one log-ellipticity domains). Note that an ill-posed inversion, inappropriate parametrization, or biased dispersion curves may prevent a good fit in the joint data space. There are some discrepancies for the fundamental mode of Rayleigh wave in the $14-18 \mathrm{~Hz}$ frequency range (Fig. 12a). Nevertheless, the modelled data still fits pretty well all individual data curves together. Further, discrepancies for the Love wave dispersion curve in the 33-41 Hz frequency range (Fig. 12d) correspond to structures at less than two meters depth, which could be ascribed to, for example shallow subsurface heterogeneity along the active seismic line. Still, our inversion is robust and does not overfit the data, supporting the validity of the self-adapting mechanism for models' complexity. To further support the resultant data fitting, we examine standardized residuals a posteriori in Supplement S5. The data variance reduction of ML and MAP models is 48 and 43 per cent, respectively, which corresponds to a fair fit.

The result of the inversion of real data is shown in Fig. 13. Posterior marginal PDFs of $v_{\mathrm{S}}$ and $v_{\mathrm{P}}$ (Figs 13a and b, respectively) are sharp in the uppermost $30 \mathrm{~m}$ of the profile, suggesting that $S$ - and $P$-wave velocities are well resolved within this depth range. The histogram for layer interfaces in Fig. 13(c) and the profiles in Figs 13(e) and (f) indicate that there are three likely interfaces at a depth of approximately 2.2, 8.5 , and $30 \mathrm{~m}$. While the first two are well resolved, the third interface's depth is uncertain (see Fig. 13e). Moreover, posterior marginal PDFs of $v_{\mathrm{S}}$ and $v_{\mathrm{P}}$ are less sharp below the depth of $30 \mathrm{~m}$ (see Figs 13a and b), meaning the resolution is reduced below the third (bottommost) interface. The velocity contrast is not sharp at the third interface (see Fig. 13a), indicating that the velocity might gradually increase with depth. Such a hypothesis is supported by comparing ML and MAP models in Fig. 13(e), where they fit in the upper part, but interfaces do not match in the profile's bottommost part. Further, AM of PDF is very smooth and gradually increases below $15 \mathrm{~m}$ depth that may also indicate a velocity gradient, although resolution is not sufficient to confirm this hypothesis. The inferred posterior marginal PDF of mass density (Fig. 13d) is unrealistic, and the ML and MAP models' $\rho$ values oscillate from one extreme to the other. From the synthetic tests (Figs 5d and 7d), we saw that this parameter could hardly be resolved, and therefore any $\rho$ value should be considered purely as the algorithm's effort 
(a)

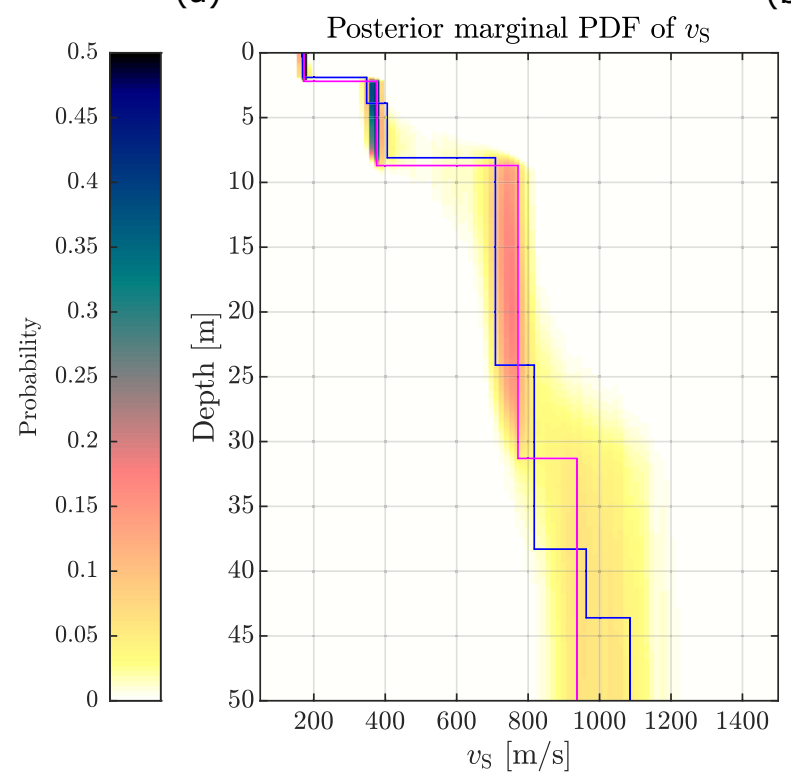

(b)

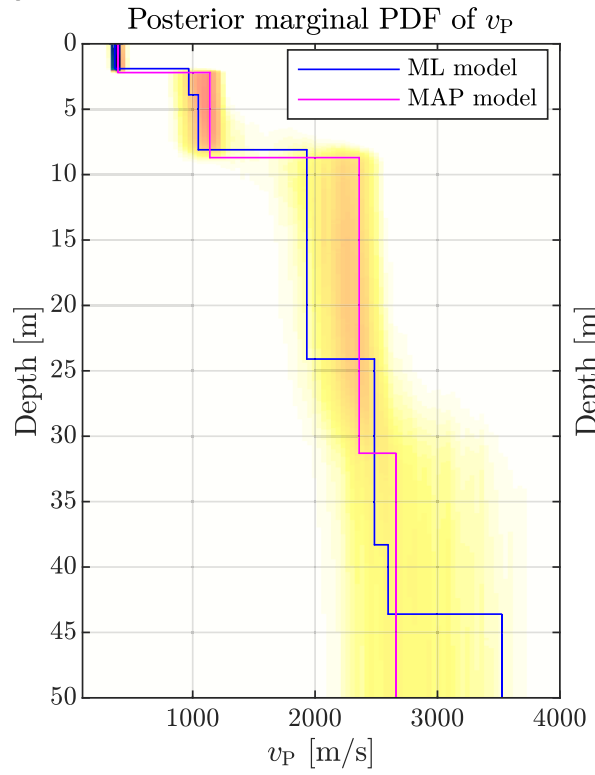

(c)

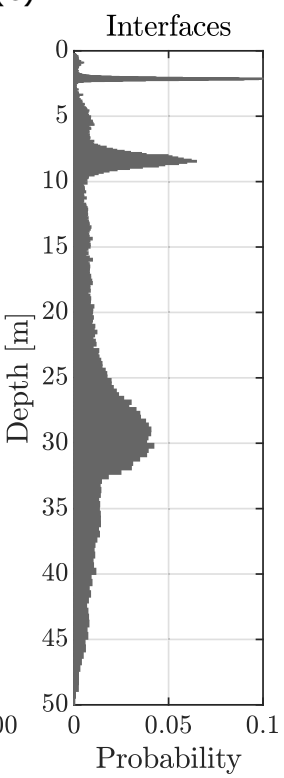

(d)

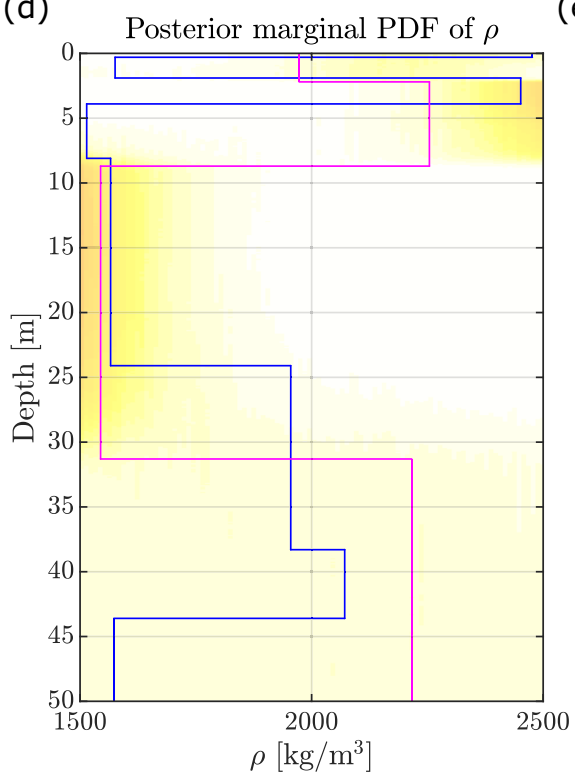

(e)

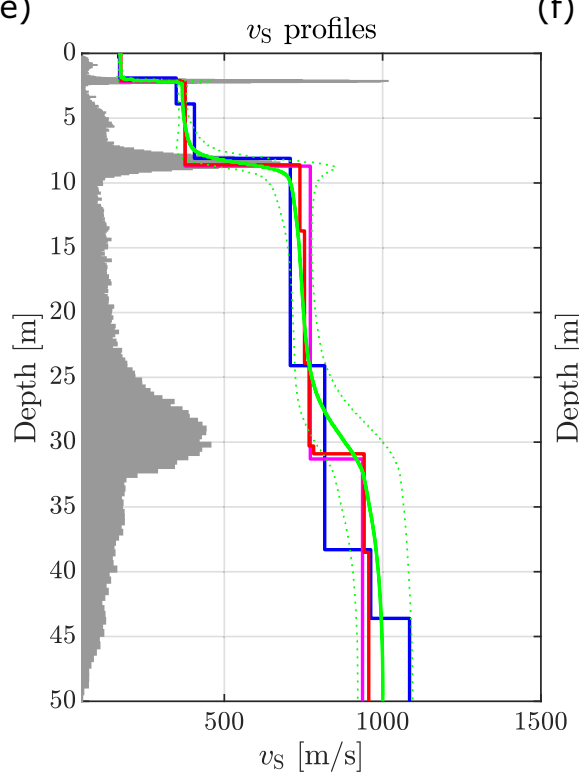

(f)

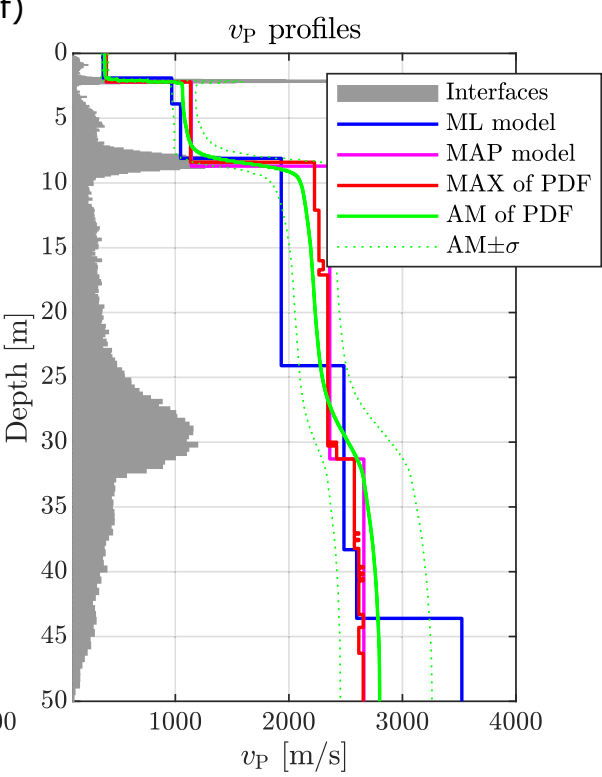

Figure 13. Inversion of real data measured at the SENGL site. The figure layout is the same as in Fig. 5.

to reduce the misfit between real data and data modelled by a simple 1-D model. This is further supported by an additional inversion with a density constraint in Supplement S4.

The ensemble of solutions exhibits a maximum probability of having four layers (Fig. 14b). It means that a velocity model having four homogeneous layers is the simplest model that can explain the real data from the SENGL site, and at the same time, the data do not (systematically) provide a more detailed resolution. In reality, however, the subsurface does not consist of homogeneous layers, and the velocity may gradually increase with depth (as speculated for the third interface). In our method, a gradient may be compensated by an increase in the number of layers (see Fig. 9), as can also be seen for the ML model (Figs 13a and e) consisting of eight layers. Nevertheless, since the gradual velocity increase at a depth of $\sim 30 \mathrm{~m}$ is uncertain, the self-adapting algorithm prefers more simple velocity models (more probable models) having four layers (see Fig. 14b).

The site-specific values of $v_{\mathrm{S} 30}$, QWL representation, and theoretical spectral amplification are useful for site characterization. The posterior marginal PDF of QWL depth (Fig. 15a) has a high resolution, highlighted by the matching QWL depth of ML and MAP models. This representation is especially useful to link features inferred in a particular depth with the experimental data's wavelength (e.g. Poggi et al. 2012). In particular, the resolution drop below the third interface at a depth of approximately $30 \mathrm{~m}$ (noted above) corresponds to a frequency of 4.4 Hz (Fig. 15a), which roughly corresponds to the lower frequency limit in the observed data (see Fig. 12). The QWL impedance (Fig. 15c) confirms that a distinct $S$-wave velocity contrast characterizes the two uppermost interfaces (\#1 and \#2), while lower values and smooth PDF 

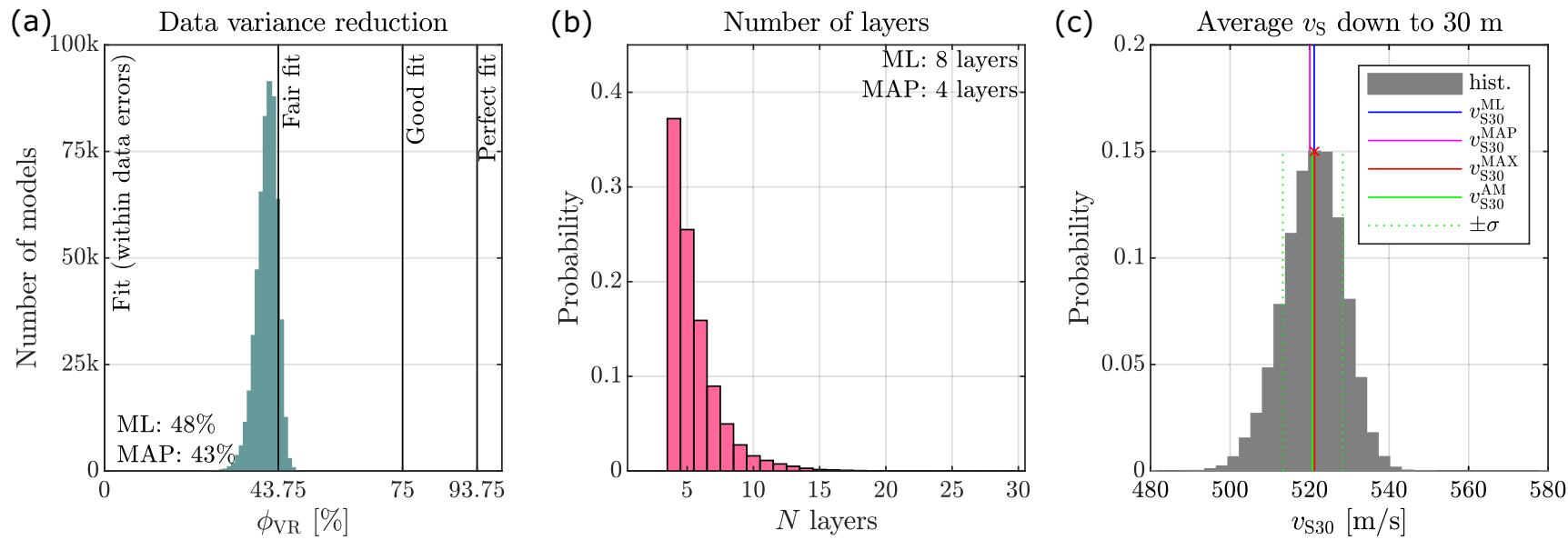

Figure 14. Histograms from the ensemble of solutions (inversion of real data measured at the SENGL site). Panels (a), (b) and (c) show histograms for data

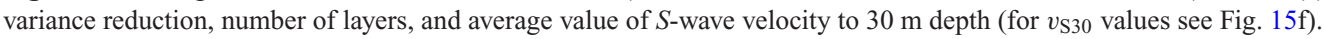

(a)

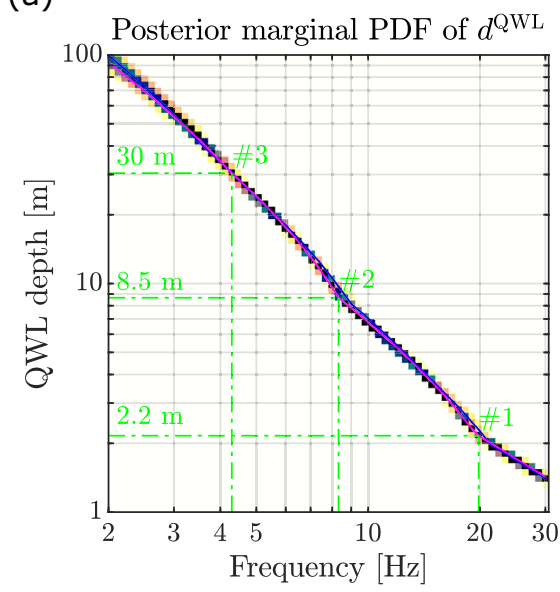

(d)

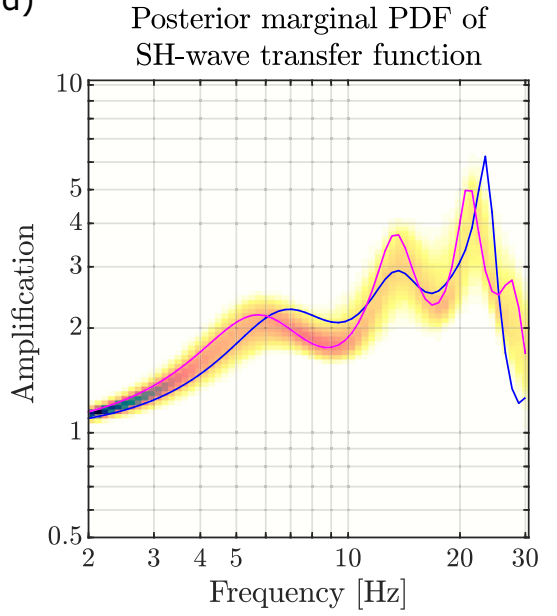

(b)

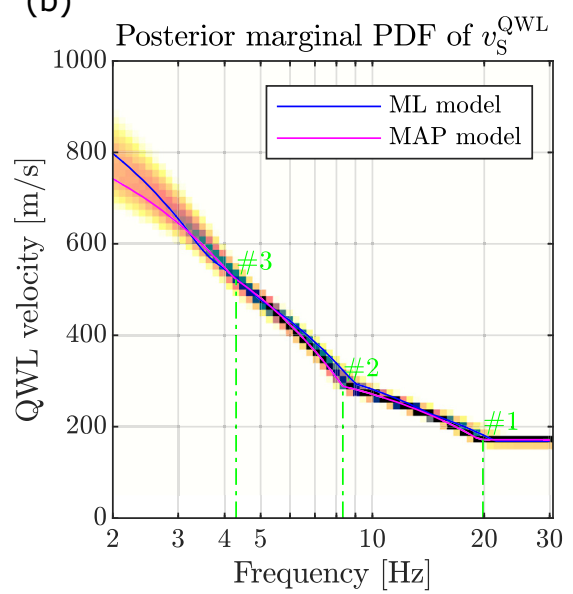

(e)

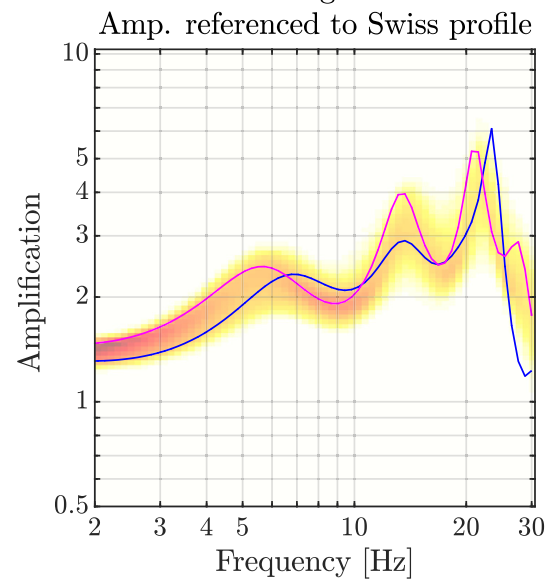

(c)

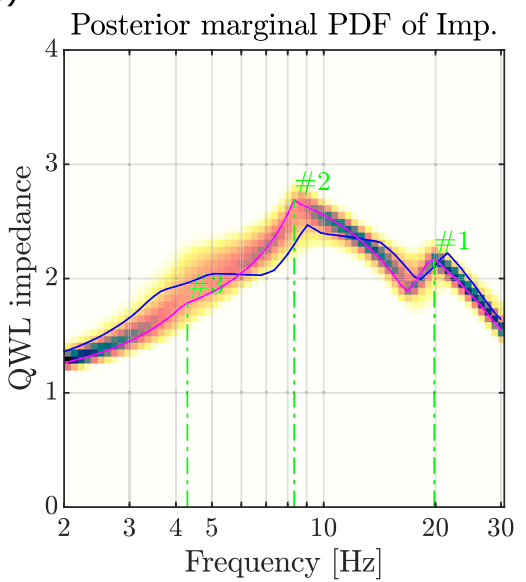

(f)

$$
\begin{aligned}
& v_{\mathrm{SL} 0}^{\mathrm{ML}}=521[\mathrm{~m} / \mathrm{s}] \quad f_{30}^{\mathrm{ML}}=4.34[\mathrm{~Hz}] \\
& v_{\mathrm{S} 30}^{\mathrm{MAP}}=520[\mathrm{~m} / \mathrm{s}] \quad f_{30}^{\mathrm{MAP}}=4.33[\mathrm{~Hz}] \\
& v_{S 30}^{\mathrm{MAX}}=521[\mathrm{~m} / \mathrm{s}] \quad f_{30}^{\mathrm{MAX}}=4.34[\mathrm{~Hz}] \\
& v_{\mathrm{S} 30}^{\mathrm{AM}}=521[\mathrm{~m} / \mathrm{s}] \\
& 513<v_{\mathrm{S} 30}^{\mathrm{AM}}<528 \\
& f_{30}^{\mathrm{AM}}=4.34[\mathrm{~Hz}] \\
& 4.3<f_{30}^{\mathrm{AM}}<4.4
\end{aligned}
$$

Figure 15. Statistics from the ensemble of solutions (inversion of real data measured at the SENGL site). The figure layout is the same as in Fig. 6. Green lines indicate properties associated with QWL depths of 2.2, 8.5 and $30 \mathrm{~m}$ (interfaces \#1, \#2 and \#3, respectively).

of impedance for the third interface (\#3) suggest, once again, a gradual velocity increase with depth. Next, the inferred $v_{\mathrm{S} 30}$ values of ML and MAP models (Fig. 15f), as well as the MAX and AM values from the ensemble of solutions (Fig. 14c), correspond to soil class B in the EC8 classification (CEN 2004). In the bottom panels of Fig. 15, we show posterior marginal PDFs of the theoretical SH-wave transfer function and theoretical amplification referenced to the Swiss profile (Poggi et al. 2011). The amplification referenced to the Swiss profile predicts the site amplification that is useful for spectral modelling (Edwards et al. 2013). However, the SENGL seismic station's actual location differs slightly from the seismic survey site, the latter being $100 \mathrm{~m}$ distant and approximately $2-3 \mathrm{~m}$ lower in altitude (see Fig. 11). We do not 
(a)

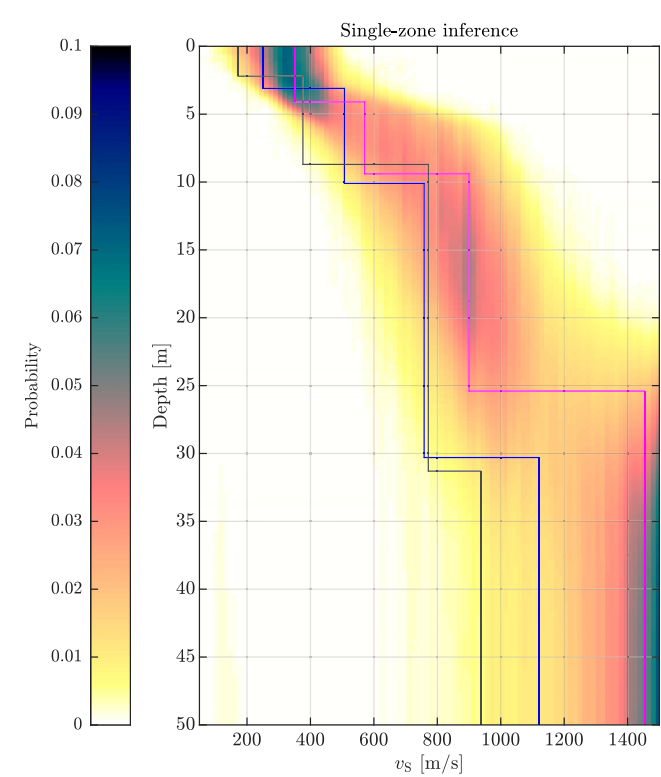

(b)

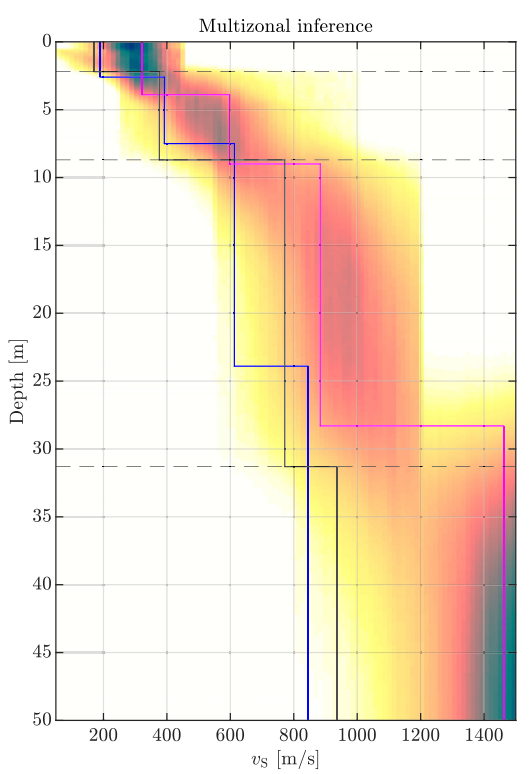

(c)

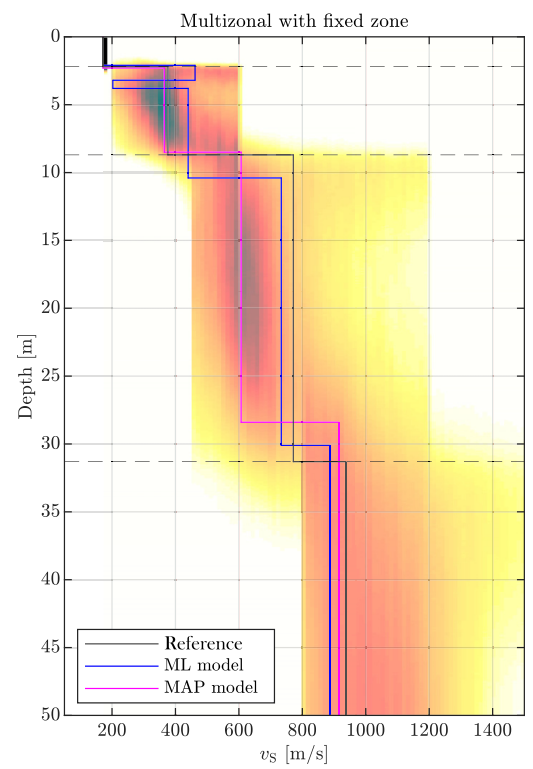

Figure 16. Inversion of the Rayleigh wave ellipticity retrieved by RayDec method (Hobiger et al. 2009) at the SENGL site. The posterior marginal PDFs of $S$-wave velocity were inferred by (a) single-zone inference, (b) multizonal inference, (c) multizonal inference with fixed velocity of the uppermost zone. The PDFs are overlayed by ML, MAP and reference models (see legend). Black dashed lines denote $z$-interfaces.

compensate for this difference here since our primary goal is simply to illustrate an application of our inversion method. Therefore, strictly speaking, the inferred amplification referenced to the Swiss profile in Fig. 15(e) is not the final theoretical prediction of the SENGL seismic station's spectral amplification.

\subsection{Inversion of the Rayleigh wave ellipticity}

In general, the dispersion curves of surface waves are not available for all the sites of interest. An inversion of the Rayleigh wave ellipticity only (data from single-station measurements) is ambiguous, but it may be beneficial if no additional data available (e.g. permanent seismic station sites, single-station extraterrestrial measurements). In this section, we test our method in cases of inversions of ellipticity data using both single-zone and multizonal model space. In particular, we use the real ellipticity data estimated by the RayDec method (Hobiger et al. 2009) at the SENGL site (Fig. 12f), while data retrieved by the MASW are excluded in this test (for the method demonstration purpose only). Since the inference based on the Rayleigh wave ellipticity is expected to have pronounced uncertainty, we take the MAP model obtained in the previous section as a reference solution (see Fig. 13a). This test is a challenge, as the ellipticity data in Fig. 12(f) are uncertain and without distinctive trough or peak; moreover, we leave the velocity gradient unconstrained, meaning that low-velocity zones are allowed to occur (i.e. $h^{\text {thr }}=h^{\max }$ ). In particular, we performed three tests: single-zone inversion, multizonal inversion, and multizonal inversion with a fixed velocity of the uppermost zone. For the multizonal inference, the model space consists of four zones corresponding to the MAP model layers obtained in the previous section. These have a thickness of 2.2, 6.5 and $22.6 \mathrm{~m}$, and the last being a half-space. For the complete list of model space thresholds, see Tables D3, D4 and D5 in Appendix D. Note that in other real multizonal applications, thicknesses of zones might be prescribed from stratigraphic logs or other external information on subsurface, and the fixed seismic velocity in the uppermost layer might be from direct measurements.

Results in terms of posterior marginal PDFs of $S$-wave velocity are shown in Fig. 16. As expected, the posterior PDFs are less sharp than in the reference solution in Fig. 13(a). Moreover, the resolution below the last interface is severely degraded (Figs 16a and b), and high $S$-wave velocity values occur because the ellipticity is essentially determined by velocity contrasts, while being weakly sensitive to its absolute value (e.g. Hobiger et al. 2013). This may also explain why the inferred $S$-wave velocities are systematically higher than in the reference solution (as the reference model in Fig. 16 is used the MAP model from Fig. 13a). On the other hand, the multizonal inference better matches the reference model when the $S$-wave velocity of the uppermost zone is fixed (see Fig. 16c), demonstrating that the inversion can effectively benefit from a priori information. However, it should be noted that incorrect prior assumptions might lead to a biased solution (see Appendix C). Further, we noted that sampling chains in the multizonal model space converging faster to a stationary state. In this particular case, the average data variance reduction after the first 1000 Markov chain steps was 79 and 86 per cent in the single-zone and multizonal inference, respectively (the final data variance reduction of the ML model is 89 per cent in all these tests). To sum up this test, the inversion of the Rayleigh wave ellipticity alone is ambiguous and interpretations from a single resultant layered model without an investigation on uncertainty should be avoided. However, the rate of velocity change with depth (i.e. pattern of the velocity profile) may be inferred and interpreted in terms of local geology. 


\section{DISCUSSION}

Observed and modelled data are subject to uncertainty originating from measurement errors (i.e. errors of dispersion and ellipticity curves) and the simplified modelling of a natural phenomenon (i.e. how close are the observed real data to the data modelled in 1-D subsurface structures). In the Bayesian inversion, we can account for these errors jointly through the data covariance matrix or vector of the data variance, see eqs (6) and (7). Unfortunately, no rigorous research on these errors has been performed in the framework of local near-surface imaging to date. Therefore, expected data errors are treated as a user-defined expert estimate in this research. These expected errors can be determined empirically during processing of raw field data.

The model space is constrained through minimal and maximum plausible values of model parameters (see Appendix D). This is a trait common to most inversion platforms in this field (e.g. layer threshold values in Geopsy). Our inversion can effectively benefit from a priori information constraining these thresholds (see Figs 7, 8 and 16); nevertheless, it is essential to set the limits of $S$ - and $P$-wave velocities as wide enough if no reliable information on the subsurface is available a priori. Too tight or wrong thresholds may lead to biased solutions neglecting the information carried by the measured data. In our approach, this issue is clearly indicated by a sharp posterior marginal PDF at the edge of the interval of plausible values (see Fig. C2). Furthermore, constraining the positive velocity gradient, as implicitly done in most inversion techniques in site characterization, significantly affects the model space itself. This can be deduced from the effective prior on the model space (see Fig. 3). Hence, these constraints should be applied only in justified cases to prevent biased solutions (e.g. when too small segments of dispersion curves or just fundamental mode is available).

The multizonal formulation allows us to include additional model assumptions based on zones thickness and zone-specific parameter thresholds. These may be based on, for example stratigraphic logs, standard penetration tests and known water table. It can improve results (see Figs 7 and 8), but one has to avoid incorrect prior assumptions that may lead to biased solutions (see Appendix C). Another advantage of the multizonal formulation is the reduction of the model space that contains too many potential non-realistic models, thus increasing its exploration efficiency. It may be especially advantageous in those inverse problems where the model space is very extensive, and the forward problem is computationally expensive to solve.

The mass density cannot be resolved from surface wave data typically collected during site characterization studies. The inferred values of $\rho$ are most likely random without any predictive value (see Fig. 5d), and any apparent resolution (e.g. Fig. 13d) is likely caused by the automatic misfit reduction due to the 1-D approximation of near-surface structures. In our method, one may simply neglect the inferred values since $S$ - and $P$-wave velocities are treated as independent from the mass density, or use a parametrization with a fixed $\rho_{i}$ by setting $\rho_{i}^{\min }=\rho_{i}^{\max }$.

One of the most innovative features of our approach is the self-adapting number of layers. Inferences with a fixed number of layers are relatively limited when exploring the model space as they may get trapped inside the local minima of the misfit function. Moreover, the 'true' number of layers is generally unknown. In our method, the random 'birth' and 'death' of layers at random depths enhance the model space exploration, while the self-adapting mechanism controls the model complexity (parsimonious transdimensional inversion). The self-adapting mechanism prefers simple models that still explain the observed data (the explicit Occam's razor; see Appendix B); hence, it avoids models with many artificial low-velocity zones. In nature, the subsurface does not consist of homogeneous layers, and seismic velocities may gradually vary with depth. In our method, the self-adapting increase of the number of homogeneous layers compensates such continuous velocity gradients (see Fig. 9), and even when the algorithm adds needed artificial layers, a potential velocity gradient might be assumed from the posterior PDF or AM of PDF. Further, the exploration algorithm is well balanced in our framework, and hence it allows a clear identification of true low-velocity zones (see Fig. 10).

Our inversion approach provides multiple indicators useful for the interpretation purpose. The sharpness of the posterior marginal PDF may serve as an indicator of the solution uncertainty and uniqueness (e.g. an increase of uncertainty with depth, ambiguous solutions, potential velocity gradients). The histogram of interface depths indicates ambiguous or certain interfaces (bins with a low or high probability, respectively), and it indicates the interface uncertainty at depth (kurtosis of the histogram around the assumed interfaces). The inferred average profile (i.e. AM of PDF) is useful for comparing with the stratigraphic profile, and it may be the representative for sites with a smooth velocity gradient. Finally, the ML model is representative in terms of the data fit while the MAP model concerning the posterior PDF; thus, a comparison between them may indicate the reliability of the inferred features (reliable where ML and MAP models coincide).

\section{CONCLUSIONS}

The joint inversion of dispersion and ellipticity curves of surface waves is a non-linear inverse problem characterized by significant inherent non-uniqueness. It can be performed in the Bayesian probabilistic framework, where posterior PDFs of model parameters are explored by a random sampling in the model space. The inferred ensemble of models can be used to construct posterior marginal 2-D histograms showing the occurrence probability of a property of interest at a particular depth.

We introduced a joint inversion technique of surface wave dispersion and ellipticity data, exploring various non-unique solutions and adapting the number of layers of the velocity models as required by the observed data. The model space is explored via the reversible jump Markov chain Monte Carlo algorithm (Green 1995), providing model samples drawn from the posterior PDF. The sampling mechanism is enhanced by using the Parallel Tempering technique (Sambridge 2014) to improve the exploration of the complex multidimensional model space. The parametrization for the variable number of layers is similar to that of Bodin et al. (2012), and the explicit Occam's razor 
controlling the number of layers follows the approach of Hallo \& Gallovič (2020). The method is rigorously formulated in a general multizonal transdimensional Bayesian framework that allows the definition of many special cases like, for example:

Our inversion technique was first validated based on synthetic tests. The former helped us to understand the general behaviour of the proposed algorithm. The inferred $S$ - and $P$-wave velocity values and interface depths satisfactorily matched the corresponding target values, and the estimated solution uncertainties followed the natural data resolution decrease with depth. We also demonstrated the self-adapting mechanism's reliability in inferring complex subsurface structures with continuous velocity gradients and low-velocity zones. Finally, we applied our approach to data obtained from active seismic survey and single-station ambient vibrations measurements at the SENGL site, central Switzerland. This test confirmed the robustness of our joint inversion algorithm when applied to multiple surface wave modes and ellipticity, and it therefore represents a valuable tool for site characterization studies with respect to the solution uncertainty and non-uniqueness (e.g. $v_{\mathrm{S} 30}$ can be given by histogram).

\section{ACKNOWLEDGEMENTS}

Foremost, MH and WI were supported through a contract with the Swiss Federal Nuclear Safety Inspectorate (ENSI). The authors thank all colleges of our home institution, Swiss Seismological Service (SED), ETH Zurich. Special thanks go to Manuel Hobiger for his valuable advice in this project's initial phase, Malcolm Sambridge for the fruitful discussions during his visit at ETH, and Paolo Bergamo and Paulina Janusz for their participation in the field measurements at the SENGL site. The authors thank Jan Dettmer and an anonymous reviewer for comments improving the quality of the manuscript. In this project, we developed a software package based on the Multizonal Transdimensional Inversion (available upon request). It uses some routines from the Parametric Slip Inversion (Hallo \& Gallovič 2020) available under the GNU General Public License and the computer package Parallel Tempering (Sambridge 2014). The latter was made available with support from the Inversion Laboratory (ilab, http://www.iearth.edu.au/), which is a program for construction and distribution of data inference software in the geosciences supported by AuScope Ltd, a nonprofit organization for Earth Science infrastructure funded by the Australian Federal Government. The Geopsy open-source software (Wathelet et al. 2020, http://www.geopsy.org/) was used for the forward computations. Author contribution statement: MH prepared the concept, designed the method, programmed the software, inferred results, and wrote the manuscript. WI prepared the concept, programmed forward problem interface, and wrote the manuscript. FP measured and analysed the real data and wrote the manuscript. DF supervised this project and wrote the manuscript. The authors declare they have no competing interests.

\section{DATA AVAiLABiLity}

The data underlying this article are available in Swiss Seismological Service (SED) at ETH Zurich (2015), at https://dx.doi.org/10.12686/SE D-STATIONCHARACTERIZATIONDB.

\section{REFERENCES}

Aki, K., 1957. Space and time spectra of stationary stochastic waves, with special reference to microtremors, Bull. Earthq. Res. Inst., 35, 415-456.

Arai, H. \& Tokimatsu, K., 2004. S-wave velocity profiling by inversion of microtremor H/V spectrum, Bull. seism. Soc. Am., 94(1), 53-63.

Arai, H. \& Tokimatsu, K., 2005. S-wave velocity profiling by joint inversion of microtremor dispersion curve and horizontal-to-vertical $(\mathrm{H} / \mathrm{V})$ spectrum, Bull. seism. Soc. Am., 95(5), 1766-1778.

Bayes, T., 1763. An essay towards solving a problem in the doctrine of chances, Phil. Trans. R. Soc. Lond., 53, 370-418.

Bodin, T. \& Sambridge, M., 2009. Seismic tomography with the reversible jump algorithm, Geophys. J. Int., 178(3), 1411-1436.

Bodin, T., Sambridge, M., Tkalčić, H., Arroucau, P., Gallagher, K. \& Rawlinson, N., 2012. Transdimensional inversion of receiver functions and surface wave dispersion, J. geophys. Res., 117, B02301, doi:10.1029/2011JB008560.

Bonnefoy-Claudet, S., Cotton, F. \& Bard, P.-Y., 2006. The nature of noise wavefield and its applications for site effects studies, Earth Sci. Rev., 79, 205-227.

Bonnefoy-Claudet, S., Köhler, A., Cornou, C., Wathelet, M. \& Bard, P.-Y., 2008. Effects of Love waves on microtremor H/V ratio, Bull. seism. Soc. Am., 98(1), 288-300.

Boore, D.M., 2003. Simulation of ground motion using the stochastic method, Pure appl. Geophys., 160, 635-676.

Borcherdt, R.D., 1994. Estimates of Site-dependent response spectra for design (methodology and justification), Earthq. Spectra, 10(4), 617-653.

CEN, 2004. Eurocode 8: Design of Structures for Earthquake Resistance - Part 1: General Rules, Seismic Actions and Rules for Buildings, EN 1998-1 edn, 229pp., European Committee for Standardization.
Cipta, A., Cummins, P., Dettmer, J., Saygin, E., Irsyam, M., Rudyanto, A. \& Murjaya, J., 2018. Seismic velocity structure of the Jakarta Basin, Indonesia, using trans-dimensional Bayesian inversion of horizontal-to-vertical spectral ratios, Geophys. J. Int., 215(1), 431-449.

D’Amico, V., Picozzi, M., Baliva, F. \& Albarello, D., 2008. Ambient noise measurements for preliminary site-effects characterization in the urban area of florence. Bull. seism. Soc. Am., 98(3), 1373-1388.

Dettmer, J., Dosso, S.E. \& Holland, C.W., 2007. Uncertainty estimation in seismo-acoustic reflection travel-time inversion, J. acoust. Soc. Am., 122, 161-176.

Dettmer, J., Dosso, S.E. \& Holland, C.W., 2010. Trans-dimensional geoacoustic inversion, J. acoust. Soc. Am., 128, 3393-3405.

Dettmer, J., Molnar, S., Steininger, G., Dosso, S.E. \& Cassidy, J.F., 2012. Trans-dimensional inversion of microtremor array dispersion data with hierarchical autoregressive error models, Geophys. J. Int., 188(2), 719734.

Dettmer, J. \& Dosso, S.E., 2012. Trans-dimensional matched-field geoacoustic inversion with hierarchical error models and interacting Markov chains, J. acoust. Soc. Am., 132, 2239-2250.

Dosso, S.E., Dettmer, J., Steininger, G. \& Holland, C.W., 2014. Efficient trans-dimensional Bayesian inversion for geoacoustic profile estimation, Inverse Problems, 30, 114018.

Dunkin, J.W., 1965. Computation of modal solutions in layered elastic media at high frequencies, Bull. seism. Soc. Am., 55(2), 335-358.

Edwards, B., Michel, C., Poggi, V. \& Fäh, D., 2013. Determination of site amplification from regional seismicity: application to the Swiss National Seismic Networks, Seismol. Res. Lett., 84(4), 611-621.

Fäh, D., Kind, F. \& Giardini, D., 2001. A theoretical investigation of average H/V ratios, Geophys. J. Int., 145(2), 535-549. 
Fäh, D., Kind, F. \& Giardini, D., 2003. Inversion of local S-wave velocity structures from average $\mathrm{H} / \mathrm{V}$ ratios, and their use for the estimation of site-effects. J. Seismol., 7, 449-467.

Ford, S.R., Dreger, D.S. \& Walter, W.R., 2009. Identifying isotropic events using a regional moment tensor inversion, J. geophys. Res., 114, B01306, doi:10.1029/2008JB005743.

Foti, S., Comina, C., Boiero, D. \& Socco, L.V., 2009. Non-uniqueness in surface-wave inversion and consequences on seismic site response analyses, Soil Dyn. Earthq. Eng., 29, 982-993.

Foti, S. et al., 2018. Guidelines for the good practice of surface wave analysis: a product of the InterPACIFIC project, Bull. Earthq. Eng., 16(6), $2367-2420$

Gallagher, K., Charvin, K., Nielsen, S., Sambridge, M. \& Stephenson, J., 2009. Markov chain Monte Carlo (MCMC) sampling methods to determine optimal models, model resolution and model choice for earth science problems, Mar. Petrol. Geol., 26, 525-535.

Gelman, A., Carlin, J.B., Stern, H.S., Dunson, D.B., Vehtari, A. \& Rubin, D.B., 2013. Bayesian Data Analysis, 3rd edn, 675pp., Chapman and $\mathrm{Hall} / \mathrm{CRC}$.

Geyer, C.J., 1991. Markov chain Monte Carlo maximum likelihood, in Proceedings of the 23rd Symposium on the Interface Computing Science and Statistics, pp.156-163, Interface Foundation, Fairfax Station, VA.

Green, P.J., 1995. Reversible jump Markov chain Monte Carlo computation and Bayesian model determination, Biometrika, 82(4), 711-732.

Green, P.J., 2003. Trans-dimensional Markov chain Monte Carlo, in Highly Structured Stochastic Systems, pp. 179-198, Oxford Univ. Press.

Hallo, M., Asano, K. \& Gallovič, F., 2017. Bayesian inference and interpretation of centroid moment tensors of the 2016 Kumamoto earthquake sequence, Kyushu, Japan, Earth, Planets Space, 69, 134.

Hallo, M. \& Gallovič, F., 2020. Bayesian self-adapting fault slip inversion with Green's functions uncertainty and application on the 2016 Mw7.1 Kumamoto earthquake, J. geophys. Res., 125, e2019JB018703, doi:10.1029/2019JB018703.

Haskell, N.A., 1953. The dispersion of surface waves on multilayered media, Bull. seism. Soc. Am., 43(1), 17-34.

Hastings, W.K., 1970. Monte Carlo sampling methods using Markov Chains and their applications, Biometrika, 57, 97-109.

Hobiger, M., Bard, P.-Y., Cornou, C. \& Le Bihan, N., 2009. Single station determination of Rayleigh wave ellipticity by using the random decrement technique (RayDec), Geophys. Res. Lett., 36(14), L14303, doi:10.1029/2009GL038863.

Hobiger, M. et al., 2013. Ground structure imaging by inversions of Rayleigh wave ellipticity: sensitivity analysis and application to European strongmotion sites, Geophys. J. Int., 192(1), 207-229.

Hobiger, M., Fäh, D., Michel, C., Burjánek, J., Maranò, S., Pilz, M., Imperatori, W. \& Bergamo, P., 2016. Site characterization in the framework of the renewal of the Swiss strong motion network (SSMNet), in Proceedings of the 5th IASPEI/IAEE International Symposium: Effects of Surface Geology on Seismic Motion, August 15-17, 2016.

Horike, M., 1985. Inversion of phase velocity of long-period microtremors to the S-wave-velocity structure down to the basement in urbanized areas, J. Phys. Earth, 33(2), 59-96.

Jaynes, E.T., 2003. Probability Theory, The Logic of Science, 727pp., Cambridge Univ. Press.

Jefferys, W. \& Berger, J., 1992. Ockham's razor and Bayesian analysis. Am. Sci., 80(1), 64-72.

Joyner, W.B., Warrick, R.E. \& Fumal, T.E., 1981. The effect of quaternary alluvium on strong ground motion in the Coyote Lake, California, earthquake of 1979, Bull. seism. Soc. Am., 71(4), 1333-1349.

Lai, C.G., Foti, S. \& Rix, G.J., 2005. Propagation of data uncertainty in surface wave inversion, J. Eng. Environ. Geophys., 10(2), 219-228.

Li, C., Dosso, S.E., Dong, H., Yu, D. \& Liu, L., 2012. Bayesian inversion of multimode interface-wave dispersion from ambient noise, IEEE J. Ocean. Eng., 37(3), 407-416.

Lunedei, E. \& Albarello, D., 2009. On the seismic noise wavefield in a weakly dissipative layered Earth, Geophys. J. Int., 177(3), 1001-1014.
Malinverno, A., 2002. Parsimonious Bayesian Markov chain Monte Carlo inversion in a nonlinear geophysical problem, Geophys. J. Int., 151, 675688

Maranò, S., Reller, C., Loeliger, H.-A. \& Fäh, D., 2012. Seismic waves estimation and wavefield decomposition: application to ambient vibrations, Geophys. J. Int., 191(1), 175-188.

Maranò, S., Hobiger, M. \& Fäh, D., 2017. Retrieval of Rayleigh wave ellipticity from ambient vibration recordings, Geophys. J. Int., 209(1), 334-352.

Maraschini, M. \& Foti, S., 2010. A Monte Carlo multimodal inversion of surface waves, Geophys. J. Int., 182(3), 1557-1566.

Metropolis, N., Rosenbluth, A., Rosenbluth, M., Teller, A. \& Teller, E., 1953. Equation of state calculations by fast computing machines, J. Chem. Phys., 21, 1081-1092.

Molnar, S., Dosso, S.E. \& Cassidy, J.F., 2010. Bayesian inversion of microtremor array dispersion data in southwestern British Columbia, Geophys. J. Int., 183(2), 923-940.

Nakamura, Y., 1989. A method for dynamic characteristics estimation of subsurface using microtremor on the ground surface, Railw. Tech. Res. Inst. Q Rep., 30(1), 25-33.

Nogoshi, M. \& Igarashi, T., 1971. On the amplitude characteristics of microtremor (part 2), J. Seismol. Soc. Jpn., 24, 26-40 (in Japanese with English abstract).

Okada, H., 2003. The Microtremor Survey Method, Geophysical Monograph Series, Vol., 12, 150pp., Society of Exploration Geophysicists.

Panzera, F., Bergamo, P. \& Fäh, D., 2020. Site Characterization Report SENGL: Engelberg (OW), 32pp., Swiss SeismologicalService (SED) at ETH Zurich, Zurich, Switzerland, (http://stations.seismo.ethz.ch).

Park, C.B., Miller, R.D. \& Xia, J., 1999. Multichannel analysis of surface waves, Geophysics, 64(3), 800-808

Parolai, S., Richwalski, S.M., Milkereit, C. \& Fäh, D., 2006. S-wave velocity profiles for earthquake engineering purposes for the Cologne Area (Germany), Bull. Earthq. Eng., 4, 65-94.

Poggi, V. \& Fäh, D., 2010. Estimating Rayleigh wave particle motion from three-component array analysis of ambient vibrations, Geophys. J. Int., 180(1), 251-267.

Poggi, V., Edwards, B. \& Fäh, D., 2011. Derivation of a reference shear-wave velocity model from empirical site amplification, Bull. seism. Soc. Am., 101(1), 258-274.

Poggi, V., Edwards, B. \& Fäh, D., 2012. Characterizing the vertical-tohorizontal ratio of ground motion at soft-sediment sites, Bull. seism. Soc. Am., 102(6), 2741-2756.

Sambridge, M., 1999. Geophysical inversion with a neighbourhood algorithm - I. Searching a parameter space, Geophys. J. Int., 138(2), 479-494.

Sambridge, M., 2014. A Parallel Tempering algorithm for probabilistic sampling and multimodal optimization, Geophys. J. Int., 196(1), 357-374.

Sambridge, M., Gallagher, K., Jackson, A. \& Rickwood, P., 2006. Transdimensional inverse problems, model comparison and the evidence, Geophys. J. Int., 167, 528-542.

Sánchez-Sesma, F.J. et al., 2011. A Theory for microtremor H/V spectral ratio: application for a layered medium, Geophys. J. Int., 186, 221-225.

Scherbaum, F., Hinzen, K.G. \& Ohrnberger, M., 2003. Determination of shallow shear wave velocity profiles in the Cologne, Germany area using ambient vibrations, Geophys. J. Int., 152(3), 597-612.

Sokos, E. \& Zahradník, J., 2013. Evaluating centroid-moment-tensor uncertainty in the new version of ISOLA software, Seism. Res. Lett., 84, 656-665.

Shynkarenko, A., Lontsi, A.M., Kremer, K., Bergamo, P., Hobiger, M., Hallo, M. \& Fäh, D., 2021. Investigating the subsurface in a shallow water environment using array and single-station ambient vibration techniques, Geophys. J. Int., under review.

[dataset] Swiss Seismological Service (SED) at ETH Zurich, 2015. The Site Characterization Database for Seismic Stations in Switzerland, ETH Zürich, doi:10.12686/sed-stationcharacterizationdb

[dataset]Swisstopo, Service géologique national, 2015. Bedrock Elevation Model. Office fédéral de topographie. https://shop.swisstopo.admin.ch/en /products/geological_models/bedrock_elevation 
[dataset]Swisstopo, Service géologique national, 2019. Geological Vector Datasets GeoCover: NM 1191 Engelberg. Office fédéral de topographie. https://map.geo.admin.ch

Tarantola, A., 2005. Inverse Problem Theory and Methods for Model Parameter Estimation, 342pp., Society for Industrial and Applied Mathematics.

Thomson, W.T., 1950. Transmission of elastic waves through a stratified solid, J. appl. Physics, 21, 89-93.

Tokimatsu, K., Kuwayama, S., Tamura, S. \& Miyadera, Y., 1991. Vs determination from steady state Rayleigh wave method, Soils Found., 31, 153-163.

Valentová, Ľ., Gallovič, F. \& Maierová, P., 2017. Three-dimensional S-wave velocity model of the Bohemian Massif from Bayesian ambient noise tomography. Tectonophysics, $\mathbf{7 1 7}, 484-498$.
Wathelet, M., Jongmans, D. \& Ohrnberger, M., 2004. Surface wave inversion using a direct search algorithm and its application to ambient vibration measurements, Near Surface Geophysics, 2, 211-221.

Wathelet, M., 2008. An improved neighborhood algorithm: parameter conditions and dynamic scaling. Geophys. Res. Lett., 35, L09301, doi:10.1029/2008GL033256.

Wathelet, M., Chatelain, J.-L., Cornou, C., Di Giulio, G., Guillier, B., Ohrnberger, M. \& Savvaidis, A., 2020. Geopsy: a user-friendly open-source tool set for ambient vibration processing. Seismol. Res. Lett., 91(3), 18781889.

Wiemer, S. et al., 2016. Seismic hazard model 2015 for Switzerland, 163pp., Swiss SeismologicalService (SED) at ETH Zurich, doi:10.12686/A2.

Yamanaka, H., Takemura, M., Ishida, H. \& Niwa, M., 1994. Characteristics of long-period microtremors and their applicability in exploration of deep sedimentary layers, Bull. seism. Soc. Am., 84(6), 1831-1841.

\section{SUPPORTING INFORMATION}

Supplementary data are available at $G J I$ online.

Figure S1.1. Convergence of the MCMC sampling procedure: Inversion of the synthetic data with the single-zone transdimensional model space (Fig. 5 in the main text). There were deployed 24 MPI nodes processing 20 parallel Markov chains per each. $X$-axis shows number of performed chain steps. $Y$-axis shows maximal data variance reduction of proposed models per each MPI node.

Figure S1.2. Convergence of the MCMC sampling procedure: Inversion of synthetic data with the multizonal transdimensional model space (Fig. 7 in the main text). There were deployed 24 MPI nodes processing 20 parallel Markov chains per each. $X$-axis shows number of performed chain steps. $Y$-axis shows maximal data variance reduction of proposed models per each MPI node.

Figure S1.3. Convergence of the MCMC sampling procedure: Inversion of the real data measured at the SENGL site (Fig. 13 in the main text). There were deployed 16 MPI nodes processing 20 parallel Markov chains per each. $X$-axis shows number of performed chain steps. $Y$-axis shows maximal data variance reduction of proposed models per each MPI node.

Figure S2.1. An example of the Gaussian random walk in the parameter space with the efficiently homogeneous prior. The initial random value is shown by the red dot. This figure is produced by the Matlab code below.

Figure S3.1. A random exploration of the union state space by the reversible-jump MCMC algorithm with the reciprocal prior. Panel (a) shows random jumps between neighbouring discrete model space states during the sampling procedure. Panel (b) shows the occurrence of the individual discrete states in the sampling chain. Indeed, the latter shows the prescribed reciprocal distribution. This figure is produced by the Matlab code below.

Figure S4.1. Fit of the real data measured at the SENGL site (black curves with errorbars) and data modelled in velocity models from the ensemble of solutions. Panels (a)-(e) show data retrieved by the MASW method (joint axes are used). Panel (f) shows data retrieved by the RayDec method (Hobiger et al. 2009) from single-station measurements of ambient vibrations.

Figure S4.2. Inversion of the real data measured at the SENGL site using constrained mass density. Panels (a), (b) and (d) show the posterior marginal PDF (colourscale) overlayed by the ML, MAP and target models (see legend). Panel (c) shows a histogram for the interface depth. Panels (e) and (f) show the inferred $S$ - and $P$-wave velocity profiles, respectively.

Figure S5.1. The covariance matrix of standardized residuals from the inversion of real data measured at the SENGL site (Fig. 12 in the main text). The standardized residuals are data misfits between the posterior predictive distribution and observed data in standardized units. Variances of standardized residuals are arranged along the descending diagonal. Data samples are discretized dispersion curves of R0, R1, R2 modes of Rayleigh waves, and L0, L1 modes of Love waves; and discretized ellipticity curve of Rayleigh waves. The frequency of discrete samples is increasing with their index within each data curve separately.

Please note: Oxford University Press is not responsible for the content or functionality of any supporting materials supplied by the authors. Any queries (other than missing material) should be directed to the corresponding author for the paper.

\section{APPENDIX A: M-H ACCEPTANCE IN THE MULTIZONAL TRANSDIMENSIONAL C A S E}

The Metropolis-Hastings (M-H) acceptance probability (Metropolis et al. 1953; Hastings 1970) is the key to ensuring that the Markov chain samples statistically follow the target posterior PDF of model parameters. Green $(1995,2003)$ extended the M-H algorithm by the multiple-state case and showed that the chain of steps in the rjMCMC converges to the transdimensional posterior PDF. This has been investigated and applied in geoscience by, for example Malinverno (2002), Sambridge et al. (2006), Bodin \& Sambridge (2009), Gallagher et al. (2009), Dettmer et al. (2010), Bodin et al. (2012) and Hallo \& Gallovič (2020). Green (1995) showed that the convergence to the 
posterior PDF $p\left(\mathbf{m} \mid \mathbf{d}_{\mathrm{obs}}\right)$ given observed data $\mathbf{d}_{\mathrm{obs}}$ occurs if the $\mathrm{M}-\mathrm{H}$ acceptance $\alpha$ of a new proposed model $\mathbf{m}^{\prime}$ given model $\mathbf{m}$ is

$\alpha\left(\mathbf{m}^{\prime} \mid \mathbf{m}\right)=\min \left(1, \frac{p\left(k^{\prime}\right)}{p(k)} \frac{p\left(\mathbf{w}_{k^{\prime}}^{\prime} \mid k^{\prime}\right)}{p\left(\mathbf{w}_{k} \mid k\right)} \frac{p\left(\mathbf{d}_{\mathrm{obs}} \mid \mathbf{m}^{\prime}\right)}{p\left(\mathbf{d}_{\mathrm{obs}} \mid \mathbf{m}\right)} \frac{q\left(\mathbf{m} \mid \mathbf{m}^{\prime}\right)}{q\left(\mathbf{m}^{\prime} \mid \mathbf{m}\right)}|\mathbf{J}|\right)$,

where $q\left(\mathbf{m}^{\prime} \mid \mathbf{m}\right)$ and $q\left(\mathbf{m} \mid \mathbf{m}^{\prime}\right)$ are proposal distributions of the forward and reverse steps, respectively, $p\left(k^{\prime}\right)$ is a prior probability of the proposed state $k^{\prime}, p\left(\mathbf{w}_{k^{\prime}}^{\prime} \mid k^{\prime}\right)$ is the prior PDF of model parameters given the proposed state $k^{\prime}$, and $p\left(\mathbf{d}_{\text {obs }} \mid \mathbf{m}^{\prime}\right)$ is the likelihood function given the proposed model $\mathbf{m}^{\prime}$. The determinant of the Jacobian $|\mathbf{J}|$ is meant to account for the volume change of the model space, and it equals unity in our case (e.g. Sambridge et al. 2006; Bodin \& Sambridge 2009; Dettmer et al. 2010).

In the case of the birth-death rjMCMC (e.g. Green 1995), the model state transitions are considered only between neghbouring states, that is so-called perturb move: $k^{\prime}=k$; birth move: $k^{\prime}=k+1$; and death move: $k^{\prime}=k-1$, where $k \equiv N$ ( $k$ is equivalent to the number of layers). The $\mathrm{M}-\mathrm{H}$ acceptance probabilities for perturb, birth, and death moves, that is $\alpha_{\mathrm{P}}, \alpha_{\mathrm{B}}$ and $\alpha_{\mathrm{D}}$ respectively, are derived from eq. (A1) by substituting $k^{\prime}$ with $k, k+1$ and $k-1$ for the respective move types. The resulting $\mathrm{M}-\mathrm{H}$ acceptance probabilities are:

$\alpha_{\mathrm{P}}\left(\mathbf{m}^{\prime} \mid \mathbf{m}\right)=\min \left(1, \frac{p\left(\mathbf{w}_{k}^{\prime} \mid k\right)}{p\left(\mathbf{w}_{k} \mid k\right)} \frac{p\left(\mathbf{d}_{\mathrm{obs}} \mid \mathbf{m}^{\prime}\right)}{p\left(\mathbf{d}_{\mathrm{obs}} \mid \mathbf{m}\right)} \frac{q\left(\mathbf{m} \mid \mathbf{m}^{\prime}\right)}{q\left(\mathbf{m}^{\prime} \mid \mathbf{m}\right)}\right)$,

$\alpha_{\mathrm{B}}\left(\mathbf{m}^{\prime} \mid \mathbf{m}\right)=\min \left(1, \frac{p(k+1)}{p(k)} \frac{p\left(\mathbf{w}_{k+1}^{\prime} \mid k+1\right)}{p\left(\mathbf{w}_{k} \mid k\right)} \frac{p\left(\mathbf{d}_{\mathrm{obs}} \mid \mathbf{m}^{\prime}\right)}{p\left(\mathbf{d}_{\mathrm{obs}} \mid \mathbf{m}\right)} \frac{q_{k+1}^{\mathrm{D}}}{q_{k}^{\mathrm{B}}}\right)$,

$\alpha_{\mathrm{D}}\left(\mathbf{m}^{\prime} \mid \mathbf{m}\right)=\min \left(1, \frac{p(k-1)}{p(k)} \frac{p\left(\mathbf{w}_{k-1}^{\prime} \mid k-1\right)}{p\left(\mathbf{w}_{k} \mid k\right)} \frac{p\left(\mathbf{d}_{\mathrm{obs}} \mid \mathbf{m}^{\prime}\right)}{p\left(\mathbf{d}_{\mathrm{obs}} \mid \mathbf{m}\right)} \frac{q_{k-1}^{\mathrm{B}}}{q_{k}^{\mathrm{D}}}\right)$,

where $q_{k}^{\mathrm{B}}$ and $q_{k}^{\mathrm{D}}$ are the probability densities of birth and death of one arbitrary VN given state $k$ (see Sambridge et al. 2006).

In the multizonal transdimensional case, the prior PDF given state $k$ in eqs (9) and (10) can be expressed as

$p\left(\mathbf{w}_{k} \mid k\right)=\prod_{i=1}^{Z}\left(p\left(\mathbf{v}_{\mathrm{S} i} \mid i\right) p\left(\mathbf{v}_{\mathrm{P} i} \mid i\right) p\left(\boldsymbol{\rho}_{i} \mid i\right)\right) \prod_{i=1}^{Z} p\left(\mathbf{h}_{i}\right)$,

$p\left(\mathbf{w}_{k} \mid k\right)=\prod_{i=1}^{Z}\left(p\left(\mathbf{v}_{\mathrm{S} i} \mid i\right) p\left(\mathbf{v}_{\mathrm{P} i} \mid i\right) p\left(\boldsymbol{\rho}_{i} \mid i\right)\right) p\left(\mathbf{h}_{k}\right)$,

where $p\left(\mathbf{h}_{k}\right)$ is the prior PDF of ln-depths of all VNi in the model vector $\mathbf{w}_{k}$ given state $k$. Moreover, the prior PDF of the ln-depth of a single VN is efficiently homogeneous and independent from others. For mathematical convenience, following Bodin \& Sambridge (2009), we assume that VNi can occur on an underlying grid of $\omega$ finite positions in the ln-depth domain (where $\omega \gg k$ ). There are $\frac{\omega !}{k !(\omega-k) !}$ possible configurations of the placement of $k$ nuclei on the underlying grid. Nevertheless, this 'grid trick' is just for convenience and there are actually infinite possible configurations. Alternatively, the same result can be achieved by rigorous derivation based on the Dirichlet distribution (Dosso et al. 2014). Then eq. (A6) becomes

$p\left(\mathbf{w}_{k} \mid k\right)=\prod_{i=1}^{Z}\left(p\left(\mathbf{v}_{\mathrm{S} i} \mid i\right) p\left(\mathbf{v}_{\mathrm{P} i} \mid i\right) p\left(\boldsymbol{\rho}_{i} \mid i\right)\right) \frac{1}{\omega !} k !(\omega-k) !$.

In the multizonal birth and death moves, one arbitrary $\mathrm{VN}$ appears or vanishes in a zone $j(j \in\{1, \ldots, Z\})$. The remaining zones collected in the set $X=\{i \in Z \mid 1 \leq i \leq Z$ and $i \neq j\}$ preserve their number of VNi (i.e. $\lambda_{i}$ ). Hence, it is convenient to modify eq. (A7) as follows

$p\left(\mathbf{w}_{k} \mid k\right)=p\left(\mathbf{v}_{\mathrm{S} j} \mid j\right) p\left(\mathbf{v}_{\mathrm{P} j} \mid j\right) p\left(\boldsymbol{\rho}_{j} \mid j\right) \prod_{i \in X}\left(p\left(\mathbf{v}_{\mathrm{S} i} \mid i\right) p\left(\mathbf{v}_{\mathrm{P} i} \mid i\right) p\left(\boldsymbol{\rho}_{i} \mid i\right)\right) \frac{1}{\omega !} k !(\omega-k) !$.

Next, we assume that the prior PDFs $p\left(v_{\mathrm{S} j} \mid j\right), p\left(v_{\mathrm{P} j} \mid j\right)$ and $p\left(\rho_{j} \mid j\right)$ of a single $\mathrm{VN}$ given zone $j$ are constant (efficiently homogeneous PDF, see eq. 11) and independent of each other. Then eq. (A8) modifies to

$p\left(\mathbf{w}_{k} \mid k\right)=\left(p\left(v_{\mathrm{S} j} \mid j\right) p\left(v_{\mathrm{P} j} \mid j\right) p\left(\rho_{j} \mid j\right)\right)^{\lambda_{j}} \prod_{i \in X}\left(p\left(\mathbf{v}_{\mathrm{S} i} \mid i\right) p\left(\mathbf{v}_{\mathrm{P} i} \mid i\right) p\left(\boldsymbol{\rho}_{i} \mid i\right)\right) \frac{1}{\omega !} k !(\omega-k) !$.

Consequently, as we use the 'birth from prior', probability densities of birth and death of one arbitrary VN in a zone $j$ are

$q_{k}^{\mathrm{B}}=p\left(v_{\mathrm{S} j} \mid j\right) p\left(v_{\mathrm{P} j} \mid j\right) p\left(\rho_{j} \mid j\right) \frac{1}{\omega-k}$,

$q_{k}^{\mathrm{D}}=\frac{1}{k}$ 
Now, we can evaluate the prior-to-proposal (PQ) ratio within $\mathrm{M}-\mathrm{H}$ acceptance terms of all move types in eqs (A2)-(A4). The PQ ratio of a simple perturb move within eq. (A2) is

$\frac{p\left(\mathbf{w}_{k}^{\prime} \mid k\right)}{p\left(\mathbf{w}_{k} \mid k\right)} \frac{q\left(\mathbf{m} \mid \mathbf{m}^{\prime}\right)}{q\left(\mathbf{m}^{\prime} \mid \mathbf{m}\right)}=1$,

because the prior PDF given state $k$ is an invariant constant in this case (i.e. $p\left(\mathbf{w}^{\prime}{ }_{k} \mid k\right)=p\left(\mathbf{w}_{k} \mid k\right)$ ), and the proposal distribution $q$ is inherently reversible as we use the Gaussian random walk, that is $q\left(\mathbf{m}^{\prime} \mid \mathbf{m}\right)=q\left(\mathbf{m} \mid \mathbf{m}^{\prime}\right)$. The PQ ratio in the $\mathrm{M}-\mathrm{H}$ acceptance of birth move within eq. (A3) is evaluated by substituting eqs (A9)-(A11) as follows:

$$
\begin{aligned}
& \frac{p\left(\mathbf{w}_{k+1}^{\prime} \mid k+1\right)}{p\left(\mathbf{w}_{k} \mid k\right)} \frac{q_{k+1}^{\mathrm{D}}}{q_{k}^{\mathrm{B}}}=\frac{\left(p\left(v_{\mathrm{S} j} \mid j\right) p\left(v_{\mathrm{P} j} \mid j\right) p\left(\rho_{j} \mid j\right)\right)^{\lambda_{j}+1}}{\left(p\left(v_{\mathrm{S} j} \mid j\right) p\left(v_{\mathrm{P} j} \mid j\right) p\left(\rho_{j} \mid j\right)\right)^{\lambda_{j}}} \frac{\prod_{i \in X}\left(p\left(\mathbf{v}_{\mathrm{S} i} \mid i\right) p\left(\mathbf{v}_{\mathrm{P} i} \mid i\right) p\left(\boldsymbol{\rho}_{i} \mid i\right)\right)}{\prod_{i \in X}\left(p\left(\mathbf{v}_{\mathrm{S} i} \mid i\right) p\left(\mathbf{v}_{\mathrm{P} i} \mid i\right) p\left(\boldsymbol{\rho}_{i} \mid i\right)\right)} \\
& \frac{\frac{1}{\omega !}(k+1) !(\omega-k-1) !}{\frac{1}{\omega !} k !(\omega-k) !} \frac{\frac{1}{k+1}}{p\left(v_{\mathrm{S} j} \mid j\right) p\left(v_{\mathrm{P} j} \mid j\right) p\left(\rho_{j} \mid j\right) \frac{1}{\omega-k}}=\frac{k+1}{\omega-k} \frac{\omega-k}{k+1}=1 .
\end{aligned}
$$

Considering that prior PDFs of separated parameters are constant (i.e. $p\left(v_{\mathrm{S}_{j}^{\prime}}^{\prime} \mid j\right)=p\left(v_{\mathrm{S} j} \mid j\right), p\left(v_{\mathrm{P}_{j}^{\prime}}^{\prime} \mid j\right)=p\left(v_{\mathrm{P}_{j}} \mid j\right)$, and $p\left(\rho_{j}^{\prime} \mid j\right)=$ $\left.p\left(\rho_{j} \mid j\right)\right)$. The PQ ratio of the death move within eq. (A4) can be expressed similarly to the birth move, as shown in eq. (A13), which also equals unity. For the interzonal move, one arbitrary VN vanishes in zone $j$ and appears in zone $j^{\prime}$ without changing its properties. The remaining zones collected in the set $Y=\left\{i \in Z \mid 1 \leq i \leq Z\right.$ and $i \neq j$ and $\left.i \neq j^{\prime}\right\}$ preserve their number of VNi. The PQ ratio in this move type (a special case of perturb move in eq. A2) can be evaluated by substituting adjusted eq. (A9) as follows:

$$
\frac{p\left(\mathbf{w}_{k}^{\prime} \mid k\right)}{p\left(\mathbf{w}_{k} \mid k\right)} \frac{q\left(\mathbf{m} \mid \mathbf{m}^{\prime}\right)}{q\left(\mathbf{m}^{\prime} \mid \mathbf{m}\right)}=\frac{\left(p\left(v_{\mathrm{S} j} \mid j\right) p\left(v_{\mathrm{P} j} \mid j\right) p\left(\rho_{j} \mid j\right)\right)^{\lambda_{j}-1}}{\left(p\left(v_{\mathrm{S} j} \mid j\right) p\left(v_{\mathrm{P} j} \mid j\right) p\left(\rho_{j} \mid j\right)\right)^{\lambda_{j}}} \frac{\left(p\left(v_{\mathrm{S}^{\prime}} \mid j^{\prime}\right) p\left(v_{\mathrm{P}^{\prime}} \mid j^{\prime}\right) p\left(\rho_{j^{\prime}} \mid j^{\prime}\right)\right)^{\lambda_{j^{\prime}}+1}}{\left(p\left(v_{\mathrm{S} j^{\prime}} \mid j^{\prime}\right) p\left(v_{\mathrm{P}^{\prime}} \mid j^{\prime}\right) p\left(\rho_{j^{\prime}} \mid j^{\prime}\right)\right)^{\lambda_{j^{\prime}}}}
$$

$\frac{\prod_{i \in Y}\left(p\left(\mathbf{v}_{\mathrm{S} i} \mid i\right) p\left(\mathbf{v}_{\mathrm{P} i} \mid i\right) p\left(\boldsymbol{\rho}_{i} \mid i\right)\right)}{\prod_{i \in Y}\left(p\left(\mathbf{v}_{\mathrm{S} i} \mid i\right) p\left(\mathbf{v}_{\mathrm{P} i} \mid i\right) p\left(\boldsymbol{\rho}_{i} \mid i\right)\right)} \frac{\frac{1}{\omega !} k !(\omega-k) !}{\frac{1}{\omega !} k !(\omega-k) !} \frac{q\left(\mathbf{m} \mid \mathbf{m}^{\prime}\right)}{q\left(\mathbf{m}^{\prime} \mid \mathbf{m}\right)}=\frac{p\left(v_{\mathrm{S}^{\prime}} \mid j^{\prime}\right) p\left(v_{\mathrm{P}^{\prime}} \mid j^{\prime}\right) p\left(\rho_{j^{\prime}} \mid j^{\prime}\right)}{p\left(v_{\mathrm{S} j} \mid j\right) p\left(v_{\mathrm{P} j} \mid j\right) p\left(\rho_{j} \mid j\right)} \frac{q\left(\mathbf{m} \mid \mathbf{m}^{\prime}\right)}{q\left(\mathbf{m}^{\prime} \mid \mathbf{m}\right)}$.

Once again, considering that prior PDFs of separated parameters are constant (i.e. $p\left(v_{\mathrm{S}_{j}}^{\prime} \mid j\right)=p\left(v_{\mathrm{S} j} \mid j\right)$, and $p\left(v_{\mathrm{S}_{j^{\prime}}} \mid j^{\prime}\right)=p\left(v_{\mathrm{S}^{\prime}} \mid j^{\prime}\right)$, and similarly for $v_{\mathrm{P} j}$ and $\rho_{j}$ ). The interzonal move is possible through perturbation of the ln-depth of a VN by the deployed Gaussian random walker. Hence, the proposal distribution $q$ in eq. (A14) is inherently reversible (symmetric at the ln-depth domain), that is $q\left(\mathbf{m}^{\prime} \mid \mathbf{m}\right)=q\left(\mathbf{m} \mid \mathbf{m}^{\prime}\right)$. Moreover, the properties of the transferred VN (i.e. $v_{\mathrm{S} j}, v_{\mathrm{P} j}$, and $\rho_{j}$ ) are preserved in the target zone $j^{\prime}$, and the PQ ratio in eq. (A14) then simplifies to

$\frac{p\left(\mathbf{w}_{k}^{\prime} \mid k\right)}{p\left(\mathbf{w}_{k} \mid k\right)} \frac{q\left(\mathbf{m} \mid \mathbf{m}^{\prime}\right)}{q\left(\mathbf{m}^{\prime} \mid \mathbf{m}\right)}=\frac{p\left(v_{\mathrm{S} j} \mid j^{\prime}\right) p\left(v_{\mathrm{P} j} \mid j^{\prime}\right) p\left(\rho_{j} \mid j^{\prime}\right)}{p\left(v_{\mathrm{S} j} \mid j\right) p\left(v_{\mathrm{P} j} \mid j\right) p\left(\rho_{j} \mid j\right)}$,

which can be determined numerically for a given pair of zones via eq. (11). Note that the PQ ratio of the interzonal move in eq. (A15) is zero if any of the model properties transferred from zone $j$ fall outside the prescribed range of the model properties in zone $j^{\prime}$.

The PQ ratios in eqs (A12)-(A15) do not include the prior probability of the model space state $p(k)$ that has been left apart in eq. (A1). The common approach is to assume uniform prior probability of the number of layers, that is $p(k)=$ const. It may be a reasonable assumption, because a prior in favour of simpler models is not necessarily required, as a model with fewer layers will automatically have a sharper posterior PDF (i.e. an objective Occam's razor; Jefferys \& Berger 1992; Malinverno 2002). However, our aim is not just the posterior PDF but also reliable and simple sampling models (see Appendix B). Moreover, the joint inversion exhibits significant inherent non-uniqueness that may complicate the posterior PDF by the presence of multiple posterior maxima. To this end, we prescribe an explicit Occam's razor for the number of layers through the prior of the model space states $p(k)$. In particular, following Hallo \& Gallovič (2020), we use the discrete reciprocal distribution expressed as follows

$p(k)=c_{\mathcal{K}} k^{-1}$,

where $c_{\mathcal{K}}$ is a normalization constant. It is a proper prior because $k$ has a positive integer value $\left(k \in Z^{+}\right)$, and it is indexing the countable collection of model states $\mathcal{K}$ as required by the definition of the union state-space by Green (2003). Then, a substitution of the first term of the $\mathrm{M}-\mathrm{H}$ acceptance of eq. (A1) has one of the following forms

$\frac{p\left(k^{\prime}\right)}{p(k)}=\frac{p(k)}{p(k)}=1$

$\frac{p\left(k^{\prime}\right)}{p(k)}=\frac{p(k+1)}{p(k)}=\frac{c_{\mathcal{K}}(k+1)^{-1}}{c_{\mathcal{K}} k^{-1}}=\frac{k}{k+1}$

$\frac{p\left(k^{\prime}\right)}{p(k)}=\frac{p(k-1)}{p(k)}=\frac{c_{\mathcal{K}}(k-1)^{-1}}{c_{\mathcal{K}} k^{-1}}=\frac{k}{k-1}$ 
Substitutions in eqs (A17), (A18) and (A19) are relevant to the $\mathrm{M}-\mathrm{H}$ acceptance probability of perturb, birth, and death moves of the birth-death rjMCMC, respectively. Note that a comparison with the classical transdimensional inversion is shown in Appendix B. Finally, the M-H acceptance probabilities for perturb $\alpha_{\mathrm{P}}$, birth $\alpha_{\mathrm{B}}$, death $\alpha_{\mathrm{D}}$ and interzonal $\alpha_{\mathrm{Z}}$ moves are expressed by substituting eqs (A12), (A13), (A15), (A18) and (A19) in eqs (A2)-(A4) as follows:

$$
\begin{aligned}
& \alpha_{\mathrm{P}}\left(\mathbf{m}^{\prime} \mid \mathbf{m}\right)=\min \left(1, \frac{p\left(\mathbf{d}_{\mathrm{obs}} \mid \mathbf{m}^{\prime}\right)}{p\left(\mathbf{d}_{\mathrm{obs}} \mid \mathbf{m}\right)}\right), \\
& \alpha_{\mathrm{B}}\left(\mathbf{m}^{\prime} \mid \mathbf{m}\right)=\min \left(1, \frac{k}{k+1} \frac{p\left(\mathbf{d}_{\mathrm{obs}} \mid \mathbf{m}^{\prime}\right)}{p\left(\mathbf{d}_{\mathrm{obs}} \mid \mathbf{m}\right)}\right), \\
& \alpha_{\mathrm{D}}\left(\mathbf{m}^{\prime} \mid \mathbf{m}\right)=\min \left(1, \frac{k}{k-1} \frac{p\left(\mathbf{d}_{\mathrm{obs}} \mid \mathbf{m}^{\prime}\right)}{p\left(\mathbf{d}_{\mathrm{obs}} \mid \mathbf{m}\right)}\right), \\
& \alpha_{\mathrm{Z}}\left(\mathbf{m}^{\prime} \mid \mathbf{m}, j, j^{\prime}\right)=\min \left(1, \frac{p\left(v_{\mathrm{S} j} \mid j^{\prime}\right) p\left(v_{\mathrm{P} j} \mid j^{\prime}\right) p\left(\rho_{j} \mid j^{\prime}\right)}{p\left(v_{\mathrm{S} j} \mid j\right) p\left(v_{\mathrm{P} j} \mid j\right) p\left(\rho_{j} \mid j\right)} \frac{p\left(\mathbf{d}_{\mathrm{obs}} \mid \mathbf{m}^{\prime}\right)}{p\left(\mathbf{d}_{\mathrm{obs}} \mid \mathbf{m}\right)}\right) .
\end{aligned}
$$

\section{APPENDIX B: IMPLEMENTED PARSIMONIOUS PRINCIPLES}

A quantification of the Occam's razor principle has a deep connection with the Bayesian inference (Jefferys \& Berger 1992). In general, the Occam's razor suggests that an explanation of facts should be no more complicated than necessary. The Bayesian inference allows multiple ways how to quantify and implement such a principle. In particular, a common implementation is the parsimonious transdimensional Bayesian inversion (Malinverno 2002) in which models with less adjustable parameters will automatically have an enhanced (sharper) posterior PDF. We designate this implementation here as the classical transdimensional inversion (Fig. B1b). Nevertheless, it is possible to implement also the second quantification that is the parsimonious selection of models. The latter chooses a simpler model for practical reasons, and it is especially beneficial when chasing a representative yet simple model. The approach introduced in this paper contains both of these quantifications together, and it is designated here as the transdimensional inversion with the reciprocal prior of $k$ (Fig. B1a).

In our application, the first quantification of the Occam's razor is a direct consequence of using the transdimensional Bayesian inversion (Malinverno 2002), where a subsurface model with fewer layers will automatically have sharper posterior PDF. This is the reason why the MAP model have usually lower number of layers than the ML model. The second quantification, designated here as an explicit Occam's razor, is implemented through the prior of the model space states $p(k)$. In particular, following Hallo \& Gallovič (2020), we use a discrete reciprocal distribution as the prior probability of $k$, which provides the parsimonious selection of sampling models. This implementation can be expressed analytically as shown in Appendix A, and it can be easily included in a computational code as shown in Supplement S3. In Fig. B1, we provide a comparison with the classical transdimensional inversion.

To compare our inversion approach with the classical transdimensional inversion, we performed an additional inversion test using classical M-H acceptance probabilities for the birth-from-prior technique (e.g. Dosso et al. 2014). We adopted the same model and data as for the synthetic test (see Table 1), the identical model space thresholds (Table D1), and exactly the same settings as of the single-zone inference. The only difference are removed terms $k /(k+1)$ and $k /(k-1)$ of eqs (13) and (14) from the computational code. The reference solution shown in Fig. B1(a) is a composite from Figs 2(a), (b), 4(f) and 5(a), while Fig. B1(b) shows results from just described inversion test. Both of these inversion tests lead to almost identical posterior marginal PDF of $v_{\mathrm{S}}$ as both include the objective (automatic) Occam's razor. However, the transdimensional inversion with the reciprocal prior of $k$ (the explicit Occam's razor) provides additionally a parsimonious selection of sampling models. It is evident when we compare the target model used for computation of the synthetic data and resultant representative ML and MAP models (see legend and annotation). This is an advantageous feature, when the selection of a representative model is required.

\section{APPENDIX C: BIASED INFERENCE DUE TO INCORRECT PRIOR ASSUMPTIONS}

The multizonal formulation allows us to take into account additional site-specific assumptions. These are included by indicating plausible zone-specific threshold values for $S$ - and $P$-wave velocities, mass density and Poisson's ratio. Additional prior assumptions may help constrain the inverse problem better and improve results, as seen in Section 5.3. However, incorrect assumptions may lead to the opposite effect and bias the solution. To further investigate this aspect, we perform two additional multizonal inferences with incorrect prior assumptions. We adopt the same model and data as for the synthetic test (see Table 1 and Fig. 4). We infer the solution in the multizonal transdimensional model space consisting of two zones and having the same burn-in and production phases as described in the main text.

For the first test (see Fig. C1), we set the depth of the $z$-interface to an incorrect value of $100 \mathrm{~m}$ (see model space thresholds in Table D6 of Appendix D). Although the resultant ML and MAP models still follow the target model (Figs C1a and b), the histogram in Fig. C1c indicates a loss of interface depth resolution. This may wrongly suggest the presence of an artificial interface or provide biased depth estimates when working with real data. In the second test (see Fig. C2), we place the $z$-interface close to the right position but specify wrong $S$ - and 
(a)
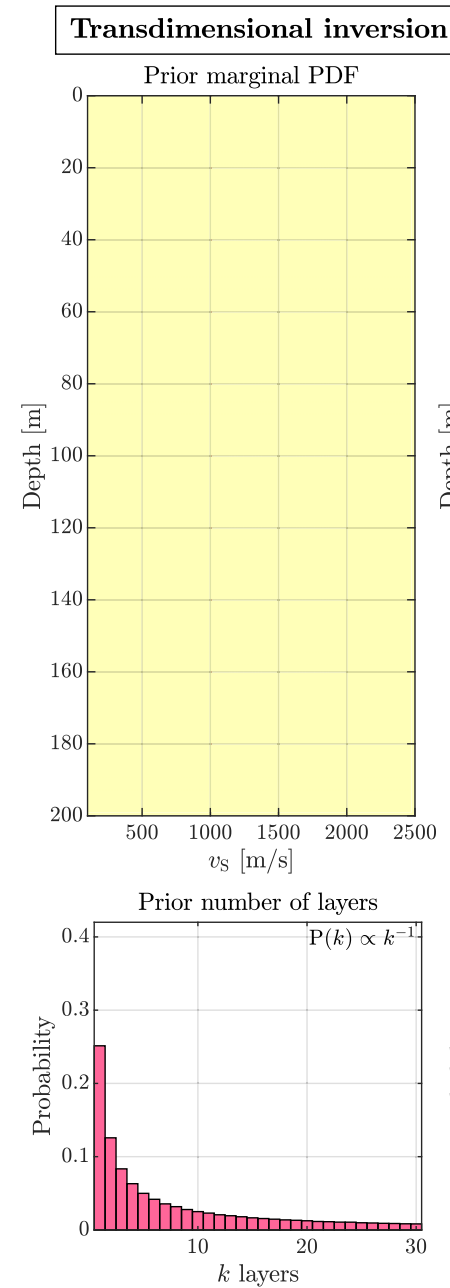

(b)
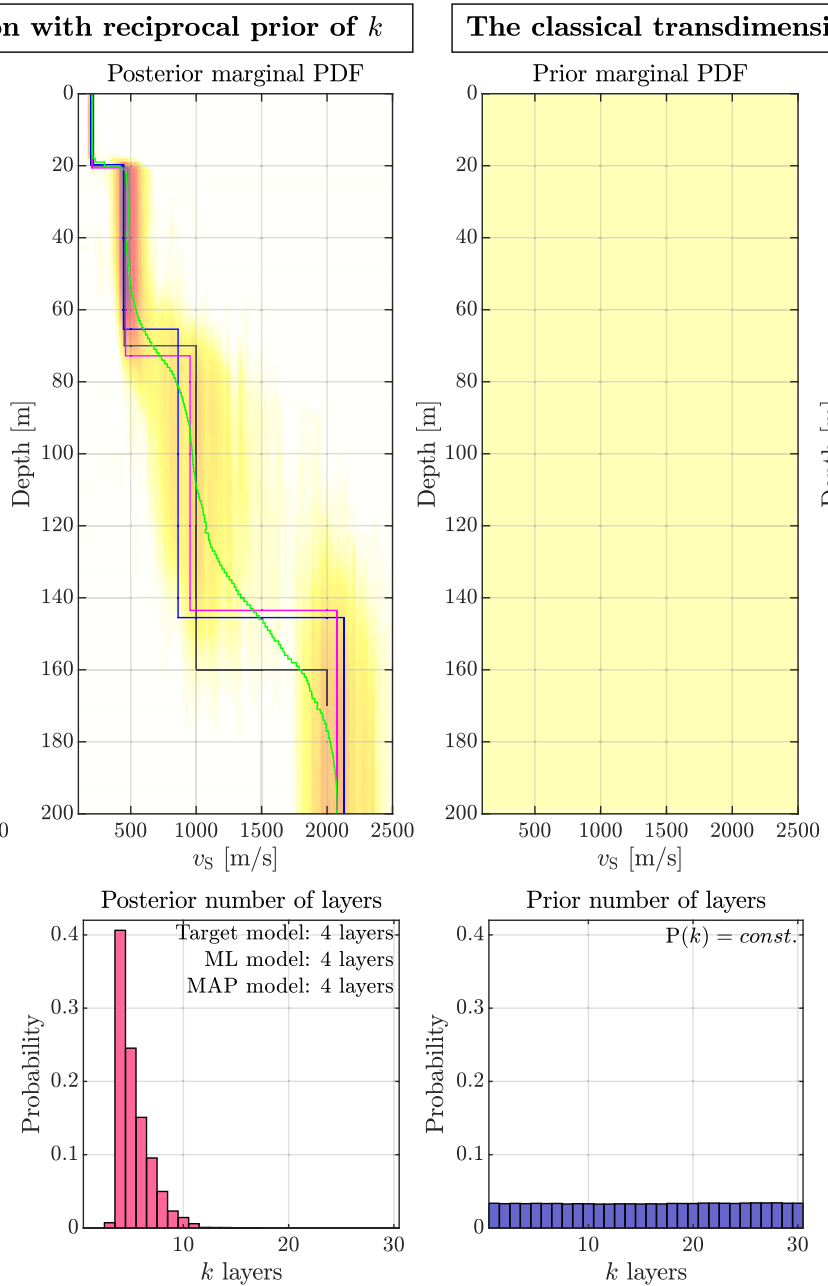

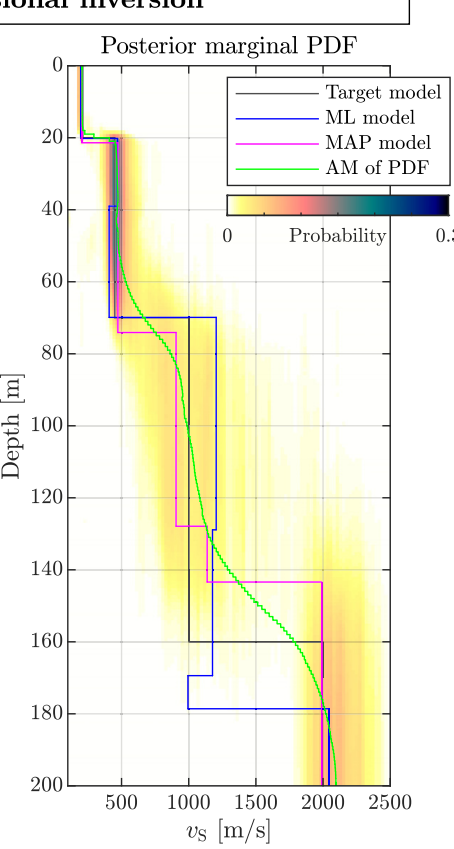

Posterior number of layers

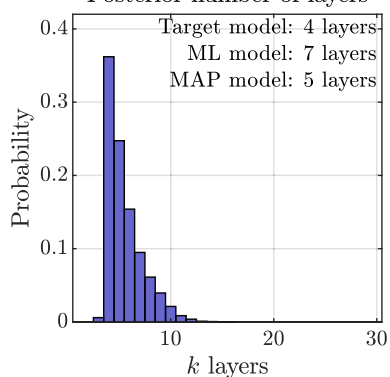

Figure B1. Comparison of the (a) implemented and (b) classical transdimensional inversion of an identical synthetic data. Both inversions assume the singlezone model space and have exactly the same settings. Upper panels show prior/posterior marginal PDFs (colourscale). Bottom panels show prior/posterior histograms of the number of layers. The target model consists of four layers and it is shown by the black line (see legend).

$P$-wave velocity thresholds in the upper zone (see model space thresholds in Table D7 of Appendix D). In particular, the true $S$-wave velocity values fall outside the model space between 70 and $154 \mathrm{~m}$ depth. These incorrect limits have a severe impact on the resultant PDF, which appears severely biased (see Fig. C2a): the systematically too low $S$-wave velocity at $70-154 \mathrm{~m}$ depth is compensated by shallower layer interfaces above and below (see Fig. C2c). Note that incorrect assumptions on the plausible range of values are identified by a sharp posterior marginal PDF at the edge of the zone-specific interval, as shown in Fig. C2(a).

\section{APPENDIX D: THRESHOLDS OF THE MODEL SPACE}

\section{APPENDIX E: LIST OF ABBREVIATIONS}

$\mathrm{AM}$ - arithmetic mean

$f-k-$ frequency-wavenumber

$\mathrm{H} / \mathrm{V}$ - horizontal to vertical

IVI - interface delimiting velocity inversion

$\mathrm{M}-\mathrm{H}$ - Metropolis-Hastings

MAP - maximum a posteriori

MASW - multichannel analysis of surface waves 

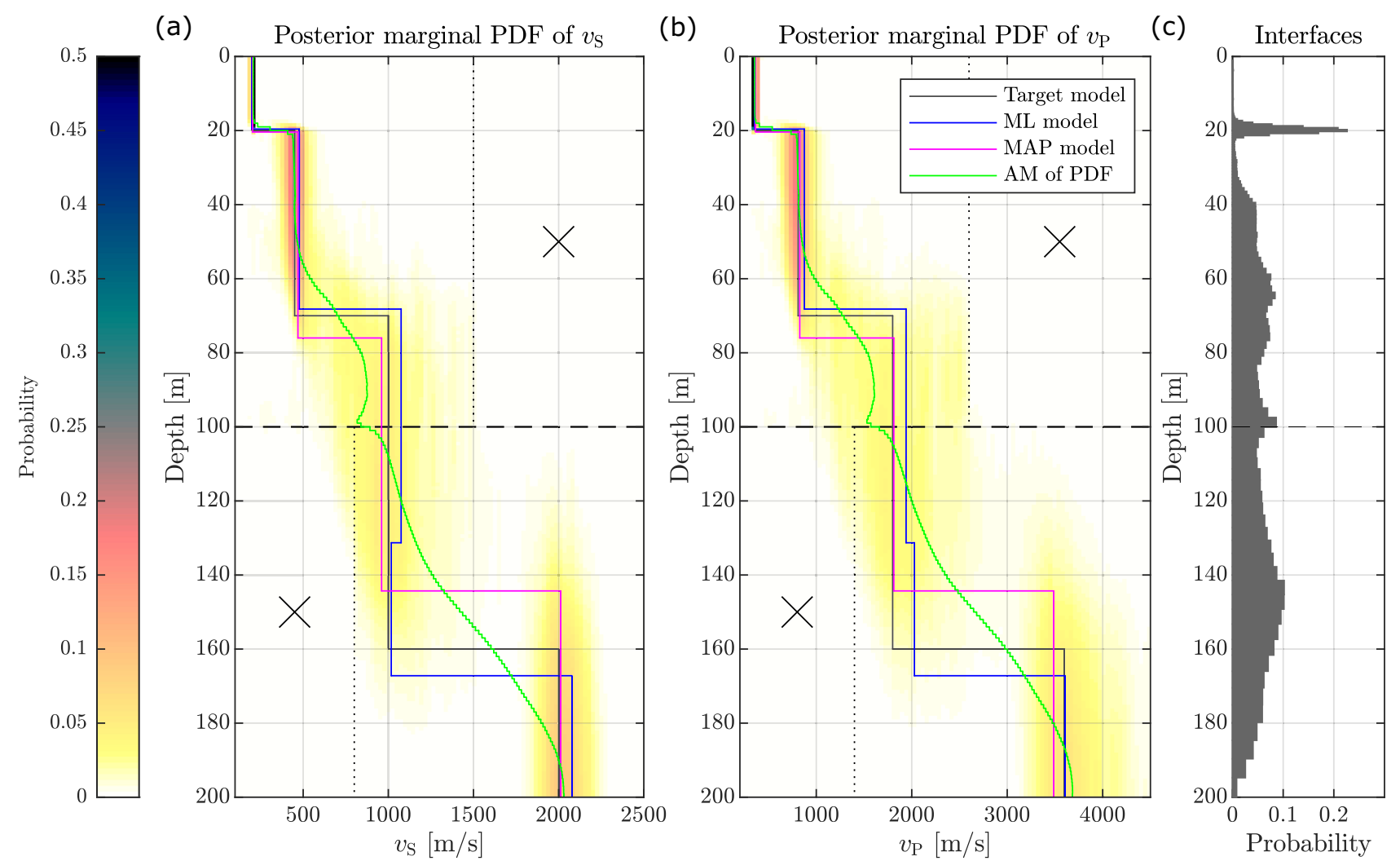

Figure C1. Multizonal inversion of synthetic data with incorrect prior assumptions on the depth of the $z$-interface (set to $100 \mathrm{~m}$ ). Panels (a) and (b) show the posterior marginal PDFs (colourscale) overlayed by the ML, MAP and target models (see legend). Panel (c) shows a histogram for the interface depth. Dotted lines show thresholds of the model space (black cross denote an area excluded from the model space).
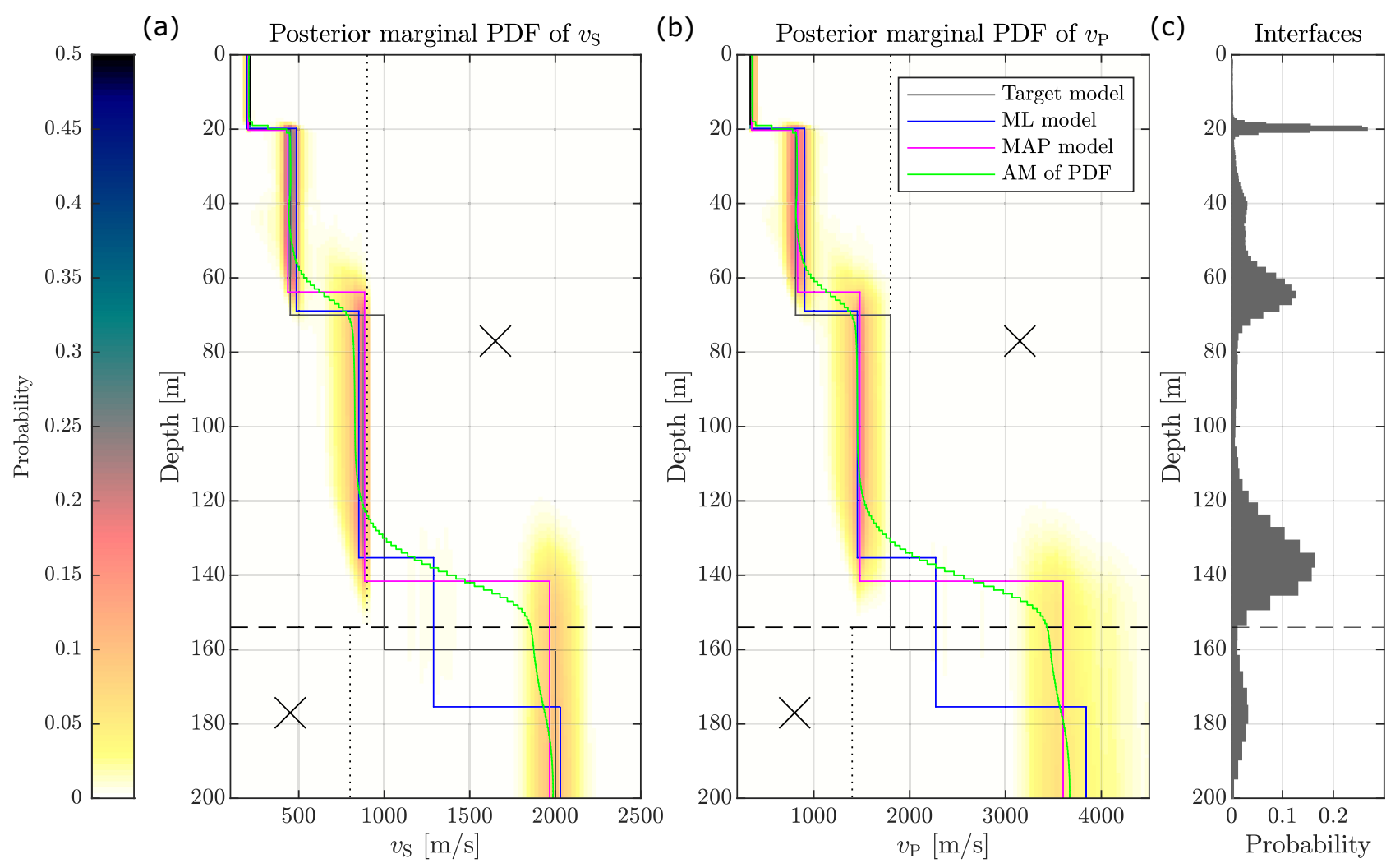

Figure C2. Multizonal inversion of synthetic data with incorrect prior assumptions on model space (vertical dotted lines). Panels (a) and (b) show the posterior marginal PDFs (colourscale) overlayed by the ML, MAP, and target models (see legend). Panel (c) shows a histogram for the interface depth. Note that true values of $S$-wave velocity are out of range of this model space at depths $70-154[\mathrm{~m}]$. 
Table D1. Inversion of synthetic data with the single-zone model space.

\begin{tabular}{|c|c|c|c|c|c|c|c|c|c|}
\hline Zone index $i$ & Depth [m] & $v_{\mathrm{S} i}^{\min }\left[\mathrm{m} \mathrm{s}^{-1}\right]$ & $v_{\mathrm{S} i}^{\max }\left[\mathrm{m} \mathrm{s}^{-1}\right]$ & $v_{\mathrm{P} i}^{\min }\left[\mathrm{m} \mathrm{s}^{-1}\right]$ & $v_{\mathrm{P} i}^{\max }\left[\mathrm{m} \mathrm{s}^{-1}\right]$ & $\rho_{i}^{\min }\left[\mathrm{kg} \mathrm{m}^{-3}\right]$ & $\rho_{i}^{\max }\left[\mathrm{kg} \mathrm{m}^{-3}\right]$ & $\eta_{i}^{\min }$ & $\eta_{i}^{\max }$ \\
\hline 1 & 0 & 100 & 2500 & 200 & 4500 & 1500 & 3000 & 0.2 & 0.4 \\
\hline
\end{tabular}

Table D2. Inversion of synthetic data with the multizonal model space.

\begin{tabular}{lccccccccc}
\hline Zone index $i$ & Depth $[\mathrm{m}]$ & $v_{\mathrm{S} i}^{\min }\left[\mathrm{m} \mathrm{s}^{-1}\right]$ & $v_{\mathrm{S} i}^{\max }\left[\mathrm{m} \mathrm{s}^{-1}\right]$ & $v_{\mathrm{P} i}^{\min }\left[\mathrm{m} \mathrm{s}^{-1}\right]$ & $v_{\mathrm{P} i}^{\max }\left[\mathrm{m} \mathrm{s}^{-1}\right]$ & $\rho_{i}^{\min }\left[\mathrm{kg} \mathrm{m}^{-3}\right]$ & $\rho_{i}^{\max }\left[\mathrm{kg} \mathrm{m}^{-3}\right]$ & $\eta_{i}^{\min }$ & $\eta_{i}^{\max }$ \\
\hline 1 & 0 & 100 & 1500 & 200 & 2600 & 1500 & 2500 & 0.2 & 0.4 \\
2 & 154 & 800 & 2500 & 1400 & 4500 & 2000 & 3000 & 0.2 & 0.4 \\
\hline
\end{tabular}

Table D3. Inversion of real data.

\begin{tabular}{cccccccccc}
\hline Zone index $i$ & Depth $[\mathrm{m}]$ & $v_{\mathrm{S} i}^{\min }\left[\mathrm{m} \mathrm{s}^{-1}\right]$ & $v_{\mathrm{S} i}^{\max }\left[\mathrm{m} \mathrm{s}^{-1}\right]$ & $v_{\mathrm{P} i}^{\min }\left[\mathrm{m} \mathrm{s}^{-1}\right]$ & $v_{\mathrm{P} i}^{\max }\left[\mathrm{m} \mathrm{s}^{-1}\right]$ & $\rho_{i}^{\min }\left[\mathrm{kg} \mathrm{m}^{-3}\right]$ & $\rho_{i}^{\max }\left[\mathrm{kg} \mathrm{m}^{-3}\right]$ & $\eta_{i}^{\min }$ & $\eta_{i}^{\max }$ \\
\hline 1 & 0 & 50 & 1500 & 100 & 4000 & 1500 & 2500 & 0.2 & 0.45 \\
\hline
\end{tabular}

Table D4. Inversion of ellipticity with the multizonal model space.

\begin{tabular}{|c|c|c|c|c|c|c|c|c|c|}
\hline Zone index $i$ & Depth [m] & $v_{\mathrm{S} i}^{\min }\left[\mathrm{m} \mathrm{s}^{-1}\right]$ & $v_{\mathrm{S} i}^{\max }\left[\mathrm{m} \mathrm{s}^{-1}\right]$ & $v_{\mathrm{P} i}^{\min }\left[\mathrm{m} \mathrm{s}^{-1}\right]$ & $v_{\mathrm{P} i}^{\max }\left[\mathrm{m} \mathrm{s}^{-1}\right]$ & $\rho_{i}^{\min }\left[\mathrm{kg} \mathrm{m}^{-3}\right]$ & $\rho_{i}^{\max }\left[\mathrm{kg} \mathrm{m}^{-3}\right]$ & $\eta_{i}^{\min }$ & $\eta_{i}^{\max }$ \\
\hline 1 & 0 & 50 & 450 & 100 & 1000 & 1500 & 2500 & 0.2 & 0.45 \\
\hline 2 & 2.2 & 250 & 1000 & 500 & 1800 & 1500 & 2500 & 0.2 & 0.45 \\
\hline 3 & 8.7 & 550 & 1200 & 1200 & 3000 & 1500 & 2500 & 0.2 & 0.45 \\
\hline 4 & 31.3 & 800 & 1500 & 1700 & 4000 & 1500 & 2500 & 0.2 & 0.45 \\
\hline
\end{tabular}

Table D5. Inversion of ellipticity with the fixed velocity in the upper zone.

\begin{tabular}{|c|c|c|c|c|c|c|c|c|c|}
\hline Zone index $i$ & Depth [m] & $v_{\mathrm{S} i}^{\min }\left[\mathrm{m} \mathrm{s}^{-1}\right]$ & $v_{\mathrm{S} i}^{\max }\left[\mathrm{m} \mathrm{s}^{-1}\right]$ & $v_{\mathrm{P} i}^{\min }\left[\mathrm{m} \mathrm{s}^{-1}\right]$ & $v_{\mathrm{P} i}^{\max }\left[\mathrm{m} \mathrm{s}^{-1}\right]$ & $\rho_{i}^{\min }\left[\mathrm{kg} \mathrm{m}^{-3}\right]$ & $\rho_{i}^{\max }\left[\mathrm{kg} \mathrm{m}^{-3}\right]$ & $\eta_{i}^{\min }$ & $\eta_{i}^{\max }$ \\
\hline 1 & 0 & 171.5 & 171.5 & 388.3 & 388.3 & 1500 & 2500 & 0.2 & 0.45 \\
\hline 2 & 2.2 & 200 & 600 & 400 & 1300 & 1500 & 2500 & 0.2 & 0.45 \\
\hline 3 & 8.7 & 450 & 1200 & 1200 & 3000 & 1500 & 2500 & 0.2 & 0.45 \\
\hline 4 & 31.3 & 800 & 1500 & 1700 & 4000 & 1500 & 2500 & 0.2 & 0.45 \\
\hline
\end{tabular}

MAX - mode (the most frequent value)

ML - maximum likelihood

MTI - multizonal transdimensional inversion

PDF - probability density function

PQ - prior-to-proposal

QWL - quarter-wavelength

RayDec - random decrement technique

rjMCMC - reversible jump Markov chain Monte Carlo

SSMNet - Swiss Strong Motion Network

VN - Voronoi nucleus 
Table D6. Inversion of synthetic data with incorrect depth of $z$-interfaces.

\begin{tabular}{|c|c|c|c|c|c|c|c|c|c|}
\hline Zone index $i$ & Depth [m] & $v_{\mathrm{S} i}^{\min }\left[\mathrm{m} \mathrm{s}^{-1}\right]$ & $v_{\mathrm{S} i}^{\max }\left[\mathrm{m} \mathrm{s}^{-1}\right]$ & $v_{\mathrm{P} i}^{\min }\left[\mathrm{m} \mathrm{s}^{-1}\right]$ & $v_{\mathrm{P} i}^{\max }\left[\mathrm{m} \mathrm{s}^{-1}\right]$ & $\rho_{i}^{\min }\left[\mathrm{kg} \mathrm{m}^{-3}\right]$ & $\rho_{i}^{\max }\left[\mathrm{kg} \mathrm{m}^{-3}\right]$ & $\eta_{i}^{\min }$ & $\eta_{i}^{\max }$ \\
\hline 1 & 0 & 100 & 1500 & 200 & 2600 & 1500 & 2500 & 0.2 & 0.4 \\
\hline 2 & 100 & 800 & 2500 & 1400 & 4500 & 2000 & 3000 & 0.2 & 0.4 \\
\hline
\end{tabular}

Table D7. Inversion of synthetic data with incorrect thresholds of model space.

\begin{tabular}{|c|c|c|c|c|c|c|c|c|c|}
\hline Zone index $i$ & Depth [m] & $v_{\mathrm{S} i}^{\min }\left[\mathrm{m} \mathrm{s}^{-1}\right]$ & $v_{\mathrm{S} i}^{\max }\left[\mathrm{m} \mathrm{s}^{-1}\right]$ & $v_{\mathrm{P} i}^{\min }\left[\mathrm{m} \mathrm{s}^{-1}\right]$ & $v_{\mathrm{P} i}^{\max }\left[\mathrm{m} \mathrm{s}^{-1}\right]$ & $\rho_{i}^{\min }\left[\mathrm{kg} \mathrm{m}^{-3}\right]$ & $\rho_{i}^{\max }\left[\mathrm{kg} \mathrm{m}^{-3}\right]$ & $\eta_{i}^{\min }$ & $\eta_{i}^{\max }$ \\
\hline 1 & 0 & 100 & 900 & 200 & 1800 & 1500 & 2100 & 0.2 & 0.4 \\
\hline 2 & 154 & 800 & 2500 & 1400 & 4500 & 2000 & 3000 & 0.2 & 0.4 \\
\hline
\end{tabular}

